\title{
On mass transport in Physarum polycephalum
}

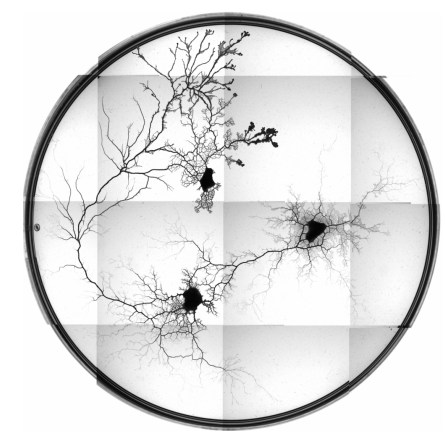

\section{Dissertation}

for the award of the degree

"Doctor rerum naturalium"

at the Georg-August-Universität Göttingen

within the doctoral degree programme

Physics of Biological and Complex Systems

of the Göttingen Graduate School of Neurosciences, Biophysics, and Molecular Biosciences (GGNB)

of the Georg-August University School of Sciences (GAUSS)

submitted by

Felix Kaspar Bäuerle

from Bühl, Germany

Göttingen, 2019 
Thesis advisory committee

Dr. Karen Alim

Biological Physics and Morphogenesis Group

Max Planck Institute for Dynamics and Self-Organization

Prof. Dr. Jörg Großhans

Zentrum Biochemie und Mol. Zellbiologie

Universitätsmedizin Göttingen

Prof. Dr. Eberhard Bodenschatz

Laboratory of Fluid Physics, Pattern Formation, and Biocomplexity Georg-August-Universität Göttingen

Members of the examination board:

Referee:

\section{Dr. Karen Alim}

Biological Physics and Morphogenesis Group

Max Planck Institute for Dynamics and Self-Organization

Co-referee:

Prof. Dr. Eberhard Bodenschatz

Laboratory of Fluid Physics, Pattern Formation, and Biocomplexity Georg-August-Universität Göttingen

Other Members of the Examination Board:

Prof. Dr. Jörg Großhans

Zentrum Biochemie und Mol. Zellbiologie

Universitätsmedizin Göttingen

Prof. Dr. Andreas Dillmann

Institut für Aerodynamik und Strömungstechnik

Deutsches Zentrum für Luft- und Raumfahrt

Dr. David Zwicker

Theory of Biological Fluids

Max Planck Institute for Dynamics and Self-Organization

Prof. Dr. Matthias Krüger

Institute for Theoretical Physics

Georg-August-Universität Göttingen

Date of the oral examination: 07. June 2019

Felix Kaspar Bäuerle: On mass transport in Physarum polycephalum, (C) April 2018 
In tiefer Dankbarkeit und

liebevoller Erinnerung an Herbert Rost.

$1939-2018$ 

The network-forming slime mold Physarum polycephalum has proven to be the epitome of self-organization. As a single cell it adapts seemingly intelligent to stimuli, integrating various inputs to create a coordinated response over an extended body plan in space and time. Most feats performed by Physarum polycephalum are linked to its morphology which is constantly reforming by transporting mass from pruning parts to growing ones. Cytoplasmic flows, the means for mass transport, are directly linked to periodic contraction patterns. Here I investigate induced mass transport in slime molds via two complementary methods: Firstly, I follow the reorganization of Physarum polycephalum networks after severe wounding and secondly I present that modulating the phase difference between harmonics increases the pumping efficiency in the slime mold when subjected to blue light.

Spatial mapping of the contraction changes in response to wounding reveal a multi-step pattern. Phases of increased activity alternate with cessation of contractions and stalling of flows, giving rise to coordinated transport and growth at the severing site. Overall, severing surprisingly acts like an attractive stimulus enabling healing of severed tubes. Furthermore I show that a modulation of the phase difference between harmonics, given cost-free constraints, directly influences the pumping efficiency by adjusting the pumps maximal occlusion. I find that the slime mold adapts its waveform accordingly when evacuating an area. It can thereby react to its environment in a self-organized fashion without changing its energy demand.

Wounding is a severe impairment of function, especially for an exposed organism like the network-forming true slime mould Physarum polycephalum and wavelike patterns driving transport are ubiquitous in living systems. The presented results may open up new venues to investigate the biochemical wiring underlying $P$. polycephalum's complex behaviours, provide a novel metric for wavelike patterns in general and demonstrate the crucial role of nonlinearities in living systems. 

PUBLICATIONS

${ }^{1}$ F. K. Bäuerle, M. Kramar, and K. Alim, "Spatial mapping reveals multistep pattern of wound healing in Physarum polycephalum," en, 50, 434005 (2017).

${ }^{2}$ F. K. Bäuerle, S. Karpitschka, and K. Alim, "Living system adapts harmonics of peristaltic wave for cost-efficient optimization of pumping performance," (submitted). 

And even when the apparatus exists,

novelty ordinarily emerges only for the man who, knowing with precision what he should expect, is able to recognize that something has gone wrong.

- Thomas S. Kuhn, The Structure of Scientific Revolutions

\section{ACKNOWLEDGEMENTS}

This work and my journey in the last three and a half years would not have been possible without the fundamental support by my brilliant colleagues, loving friends and caring family. It is too to name everybody that accompanied me and I suspect that some might be left unmentioned while they actually deserve an acknowledgement. So firstly, a heartfelt thank you to all of you for sharing this time with me, I would not have done it without you.

But undoubtedly some people shaped my work and what I can present here more profoundly than others. Above all my supervisor Karen Alim who guided and taught me about the fascination slime mold. All-the-while she built a research group that I had the honor of watching prosper under her guidance, my deep gratitude goes to you for your compelling support and endless energy throughout my $\mathrm{PhD}$ time.

For guiding my scientific road when it felt more like a rubble path I want to thank Eberhard Bodenschatz and Jörg Großhans as dear members of my thesis advisory board. Thank you Natalie Andrew for teaching me just how much one can see when staring at a video for long enough - I appreciate your patience - and your continuous support throughout the years.

Furthermore I want to thank the group and in particular Mirna Kramar, Jean-Daniel Julien, Estefania Vidal and Jason Khadka for all your questionable jokes. Nico Schramma and Noah Ziethen, thank you for being patient with me during your Bachelor time, I learned alot and am still learning from our discussions. Thank you Anne Weber for the support in the lab and keeping Physarum happy for us.

I want to thank Jana Lasser, Filippo Cosi, Shama Sograte, Laura Turco and Diemut Regel for inspiring walks in the woods as well as Holger Nobach and Christian Westendorf for frequent and fruitful input to my research. 
I deeply appreciate the support I received from the IMPRS-PBCS, as well as GGNB in general. Furthermore I want to extent my thanks to PhDnet and in particular the Survey Group 2018 for a collaboration on a matter that became close to my heart.

Lastly I want to thank the people that supported me - or distracted me well-intentioned - outside of the research community. Heartfelt thanks to my family and especially so to Lukas Bäuerle, Veronika Bäuerle, Martin Bäuerle, Barbara Rost and Herbert Rost. And thank you dear flatmates Karl, Till, Jana, Tini and Madlen -, Anna Bueble, fellow Unicantees and friends for putting up with my slime mold stories. 
CONTENTS

i introduction 1

1 motivation 3

2 introduction 7

2.1 Physarum polycephalum as a model organism . . . . . . . . . . 7

2.1.1 Contractions and shuttle streaming . . . . . . . . . 9

2.2 Phototaxis and photomorphogenesis of Physarum . . . . . . . . 14

2.2.1 Photomorphogenesis . . . . . . . . . . . . . 14

2.2.2 Phototaxis .................... . . 16

2.3 Peristaltic pumping theory . . . . . . . . . . . . . . . 19

2.3.1 Geometry and boundary conditions . . . . . . . . . . 21

2.4 Scope of the thesis . . . . . . . . . . . . . . . 24

ii materials and methods 25

3 laboratory materials and methods 27

3.1 Media recipes . . . . . . . . . . . . . . . . 27

3.2 Physarum polycephalum culture ... . . . . . . . . . . 29

3.3 Microscope setup and data acquisition . . . . . . . . . . . . . . 31

3.3 .1 Blue light stimulation . . . . . . . . . . . . . 32

3.3 .2 Cutting plasmodial tube .............. 33

4 computation and analysis methods $\quad 35$

4.1 Code handling . . . . . . . . . . . . . . . . 35

4.2 Network extraction . . . . . . . . . . . . . . . 37

4.3 Signal processing on slime mold contractions . . . . . . . . 40

4.4 Phase difference determination . . . . . . . . . . . . . 42

iii results $\quad 45$

5 spatial mapping reveals multi-step pattern of wound healing in physarum polycephalum 47

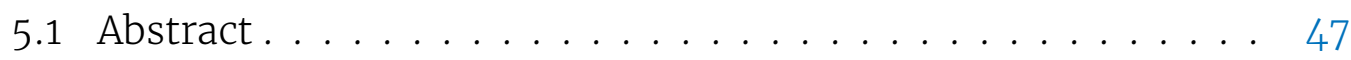

5.2 Introduction . . . . . . . . . . . . . . . . 47

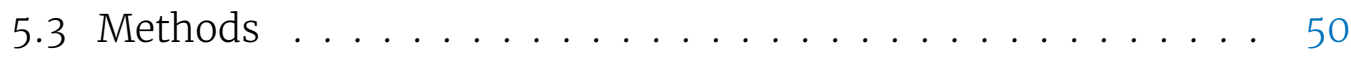

5.3 .1 Culturing and data acquisition . . . . . . . . 50

5.3.2 Comprehensive network-based contraction analysis . . 51 
5.4 Results . . . . . . . . . . . . . . . . . . . 54

5.4.1 Wounding induces fan growth at cut site . . . . . . . 54

5.4.2 Spatial mapping reveals localized stalling . . . . . . 54

5.4.3 Fan growth phase coincides with stable network-spanning contractions . . . . . . . . . . . . . 56

5.4.4 Stalling and fan growth periods are bridged by distinct transition periods . . . . . . . . . . . . . 57

5.4.5 Fan creation and stalling is reproducible for complete

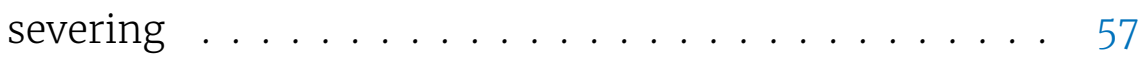

5.5 Discussion . . . . . . . . . . . . . . . 60

6 living system adapts harmonics of peristaltic wave for costefficient optimization of pumping performance 63

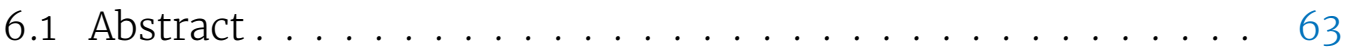

6.2 Introduction . . . . . . . . . . . . . . 63

iv discussion and outlook $\quad 75$

7 discussion and outlook 77

V appendix 81

a appendix 83

a.1 Material list . . . . . . . . . . . . . . . . . . . . 83

a.2 Agar overlay light stimulus . . . . . . . . . . . . . . . 85

a.3 Hilbert transformation . . . . . . . . . . . . . . . . 85

a.4 Kymograph based analysis . . . . . . . . . . . . . . . 86

a.5 Supplemental material for 'Living system adapts harmonics of peristaltic wave for cost-efficient optimization of pumping performance' ................... . . 87

a.5.1 Phase difference determination . . . . . . . . . . . 87

a.5.2 Physiological parameters . . . . . . . . . . . . . 88

a.5.3 Time development of experiments . . . . . . . . . . 91

a.5.4 Spatial wave patterns . . . . . . . . . . . . . . . . 92

a.5.5 Shells of revolution: Strain-displacement relations and energy considerations for an peristaltically contracting tube . . . . . . . . . . . . . . . . . 92

a.5.6 Perturbative approach to peristalsis in elastic tubes . . 96 a.6 Wound healing: List of cutting experiments . . . . . . . . . 99

a.7 Wound healing: Supplemental Figures . . . . . . . . . . . . . 121

a.8 Wound healing: Supplemental Movies . . . . . . . . . . . . 125 
bibliography 

Part I

INTRODUCTION 

Finding solutions to complex tasks is an ability we as humans quite often regard as a key distinction between us and other lifeforms. From seemingly simple tasks such as efficiently conveying information through spoken language to collective abilities such as producing and distributing food effectively in a growing population: we all solve challenging tasks daily, sometimes effortlessly, sometimes with frustration.

This skill which we commonly call intelligence is widely regarded as a feature of our brain ${ }^{1}$. Billions of neurons in an interconnected network create thoughts and store information which we may use to find the next formidable chess move or to buy groceries for the week. Animals may have similar abilities, but often lack the potential to extrapolate in time, do not exercise social learning, or fail at increasingly abstract challenges. Cognition as a whole still remains a mystery to researchers, but it is widely regarded as an emergent property of the complex system that is our brain.

In the year 2000 the researchers Toshiyuki Nakagaki, Hiroyasu Yamada and Ágota Tóth challenged this concept of intelligence in a fundamental manner ${ }^{2}$. They found that the single celled amoeboid Physarum polycephalum is able to find the shortest path through a maze. By connecting two food sources through a maze efficiently the slime mold is able to find a solution to the traveling salesmen problem. The problem is still a matter of active research and regarded in numerous applications such as connecting two computers via the internet for a fast connection, or the construction of an efficient road network.

Since the findings by Nakagaki et al. numerous so-called 'intelligent' behaviours of the slime mold have been uncovered. From building efficient transport networks 3 to solving complex nutritional challenges ${ }^{4}$ Physarum polycephalum seems to keep up rather well with our own abilities. Furthermore it anticipates periodic events in time ${ }^{5}$, is able to use external memory to navigate in space ${ }^{6}$ and exhibits habituation to remember cues over time ${ }^{7}$. The slime mold excels in various challenges and finds efficient solutions to keep on thriving all the while lacking any kind of organ or cell-cell interaction to compute a solution. How is a single celled organism able to 
integrate all of the information it collects and form a coherent response? What kind of local rules in the slime mold result in 'intelligent' behaviour and can we transfer these insights to assist technological progress in general?

To answer these questions we need to isolate principle mechanisms in Physarum polycephalum and understand their interactions. Throughout the varied investigations of slime mold behaviours, one measure for 'intelligence' has dominated - its dynamic morphology. Researchers commonly draw conclusions from, for example, the topology of the constructed network, the growth rate following a stimulus, or the spatial mass distribution after a challenge is solved. In order for Physarum polycephalum to adapt its morphology, and thereby solve the experimental challenges, it needs to move its own body mass from one location to another.

Physarum polycephalum uses a combination of peristaltic contractions which drive fluid flow, a gel-or-solification of its cytoplasm, and biochemical signals to drive mass transport. The slime mold rearranges the position of its protruding front to a favorable site, prunes unnecessary or endangered tubes, and reinforces crucial transportation routes. Each part follows local adaptation rules while influencing more distant parts primarily via the fluid flow. But, while the behavioural outcome has been studied extensively, research on the mass transport mechanism is in its infancy. Theoretical models are being developed more frequently, e.g. for peristaltic pumps, mechanochemical models of advected signals or for gel-sol transfers, and coupled contraction oscillators, but published experiments on the matter are scarce.

Physarum polycephalum as a model organism fascinates researchers across a plethora of research fields, from biology, biochemistry, physics to computer sciences, and the general public alike. It combines physical principles from hydrodynamics, network dynamics, or viscoelastic mechanics with a sophisticated biological actor. Insights are readily transferable to other fields like soft robotics, medicine, fluid dynamics, collective behaviour, future mobility or neuroscience. At the same time it shows aspects with a baffling simplicity and continues to inspire ground-breaking research. With this thesis I advance understanding of the principle mass transport mechanisms in slime mold networks, helping to uncover the 'intelligent' problem solving nature of Physarum polycephalum. The insights I present here combine the interplay of fluid dynamics driven by a biochemical contractile apparatus, spatial and temporal patterns in stimulated Physarum 
polycephalum networks, new ways of experimental procedures and of comprehensive data analysis, and novel measurable parameters for oscillatory systems in general. 



\subsection{Physarum polycephalum as a model organism}

The subject of this work, Physarum polycephalum has come a long way as a model organism in the last two centuries ${ }^{8-11}$. Its natural habitats are moist forests and is found there most commonly either in its fruiting stage or as a plasmodium, see Figure 1. The former grants Physarum polycephalum its name as it produces spore-carrying heads once it is set to end its plasmodial growth - the multi-headed slime mold. In its plasmodial stage true slime molds grow as an often yellow acellular - meaning unicellular with multiple nuclei - mass feeding on bacteria and decomposing plant matter. It remains a single cell while growing from micrometer sizes up to meter scales, even earning it an entry into the Guinness Book of Records with a recorded covered area of $5.54 \mathrm{~m}^{212}$. The usually diploid plasmodium can form sexually between two amoebas, albeit with more than 15 different mating types. Amoebas are most commonly haploid, can be seen as a gamete type cell and form a new chromosome set when mating, but can also develop asexually into a haploid plasmodium. Amoebas themselves hatch from the formerly mentioned haploid spores completing the cell cycle. At the plasmodial and the amoebal stage Physarum polycephalum can undergo an additional developmental sidestep to create a dormant stage called sclerotia or cysts respectively. In each of them the slime mold survives in dry and starved conditions for a prolonged time and can readily return to the respective starting stage when the conditions improve.

Even though the name slime mold indicates a relationship to fungi, they are actually part of the amoebozoa major taxonomic group and as closely related to animals as they are to fungi $[8,13]$. They branched off into their own clade from the better known cousins early in the eukariotic family tree indicating their exotic nature. A prominent closely related species to Physarum polycephalum includes Dictyostelium discoideum, which is known as the 'social' amoeba bridging single celled and multicellular developmental stages ${ }^{14,15}$. It has been studied extensively for its chemotaxis where some insights can be transferred to Physarum polycephalum ${ }^{16}$, but has oth- 


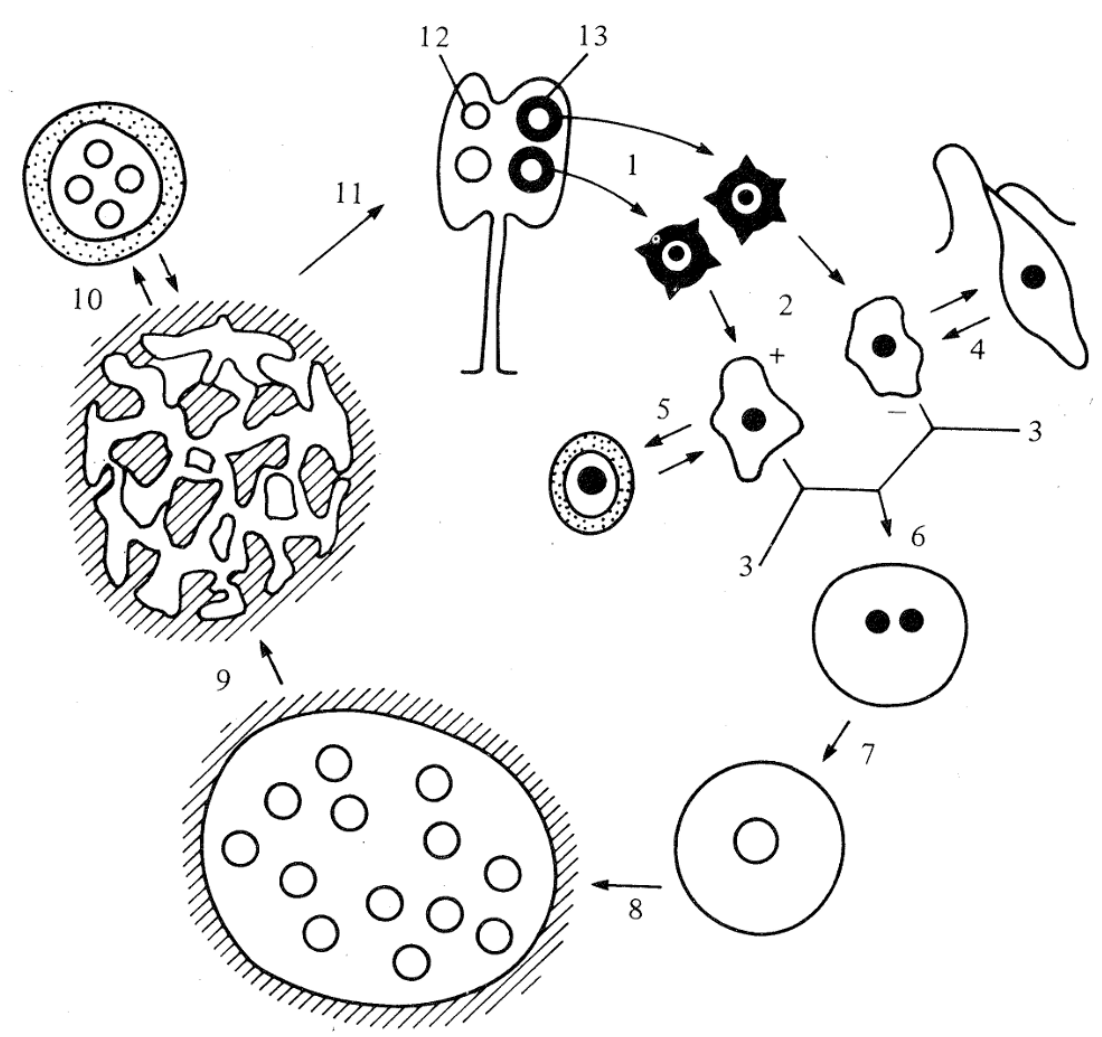

Figure 1: Lifecycle of Physarum polycephalum with all its known developmental stages. Spores (1) can hatch into amoebas (2) with complementary mating types (+ and -) and can either proliferate (3) or differentiate into a microcyst (5) or a flagellate (4) which enables it to swim in wet conditions. When fusing (6) a zyogte (7) is formed which transforms into a plasmodium (8) and can grow into characteristic network shapes (9). When starved the plasmodium can either form sclerotia (10) to stay dormant or differentiate into a sporangia (11). The pre-spores (12) in the characteristic heads go through meiosis to create new spores (13) completing the cell cycle. Figure from 'Developmental Biology of Physarum' by Helmut W. Sauer, reproduced with permission of The Licensor through PLSclear. 
erwise found its own solutions to prosper. In the same clade as Physarum polycephalum the myxogastria, are species like Badhamia utricularis or Fuligo septica which show similar oscillatory behaviour to Physarum and are recently approached quantitatively in a similar manner to Physarum polycephalum $^{17}$. Whether they prove to be just as 'intelligent' remains to be discovered.

To grow from a newly formed microplasmodium to a meter-sized network slime molds need to keep their body plan connected and information distributed all the while moving towards more fertile ground: a remarkable task for a single cell and the epitome of self-organization. When growing in the plasmodial stage Physarum polycephalum has a synchronous mitosis regardless of its size ${ }^{8,18}$. Nuclear division occurs predictably every $8 \mathrm{~h}$ giving rise to millions of nuclei while keeping a single cell wall. Likewise when well fed the growth curve in weight follows the mitosis rate. In a soft and wet environment with a food source, e.g. an agar dish with oat flakes, Physarum polycephalum will first grow isotropically forming a disklike body ${ }^{19}$. After some time the slime mold starts a foraging behaviour growing fronts in random directions when no stimulus is given. Each foraging front has a fan-like structure with a continuous gel-sol mix in the periphery, followed by fine periphery-oriented tubes and lastly leaving an isotropic network in the tail, see Figure 2. The density of the trailing network depends on the level of starvation: older networks tend to form fans with a tighter opening angle leading to more extended, but less dense networks ${ }^{18}$. When growing the trailing tail is often pruned by the slime mold turning growth into a migration. It can reach speeds of up to $1 \mathrm{~mm} \mathrm{~s}^{-120}$ reinforcing their evolutionary distance from fungi by their behavioural differences. When food sources are found scattered randomly it interconnects these food sources efficiently pruning unnecessary tubes while keeping crucial and fail-safe connections ${ }^{2}$. All in all it employs a robust hunt for nutrients by foraging for new food sources and depleting the old ones in an efficient manner.

\subsubsection{Contractions and shuttle streaming}

Each tube is made up of an inner endoplasmic and an outer ectoplasmic $\operatorname{part}^{8}$. The ectoplasm acts as an active contractile gel which pumps the endoplasm through the whole network. Structurally the ectoplasm is a combination of an invaginated cell wall and connecting microfilament bun- 


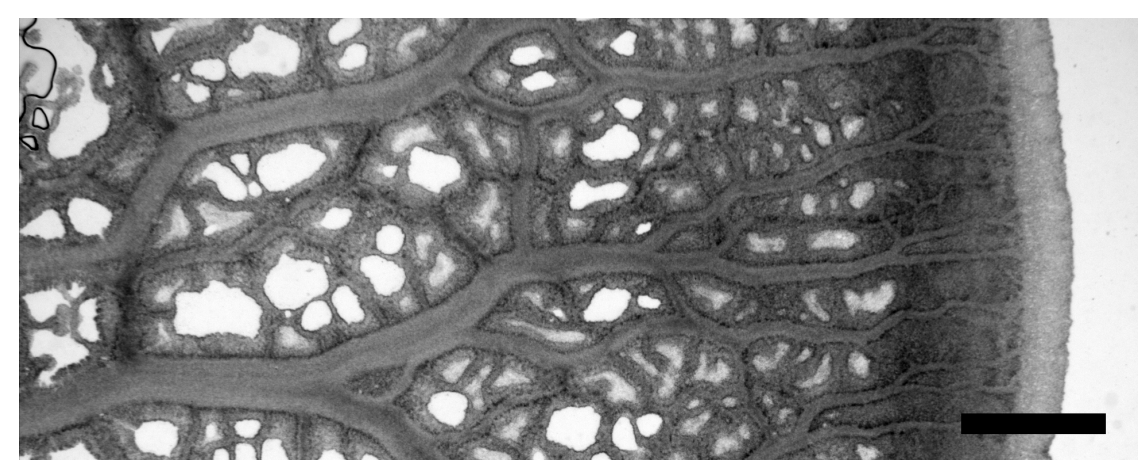

Figure 2: Fan structure in a foraging front. The periphery has an interconnected gel-sol mix followed by fine periphery-oriented tubes and an isotropic network in the tail. Agar-overlay experiment, scalebar $=1 \mathrm{~mm}$. Used with permission from Natalie Andrew.

dles, see Figure 3. The microfilaments consist of actomyosin which associate and dissociate periodically to create a contraction-relaxation cycle. Here intracellular calcium is known to play an important role, e.g. because there is a coinciding concentration cycle, calcium is bound in the myosinII motor cycle and the contraction state can be altered by adding a calcium solution $^{21-23}$.

At the same time the ectoplasmic walls are constantly reorganizing. When the microfilament bundles dissociate the monomeric actin can be capped by actinin a highly abundant protein which keeps actin from repolymerizing ${ }^{24}$. If no nucleation points exist closeby the actin-actinin complex can be carried away through the endoplasmatic flow to build up new microfilaments elsewhere. The actin concentration in the endoplasm is actually over the critical polymerization concentration and only stays in the sol phase by polymerization inhibitors. The constant reorganization is vital for Physarum polycephalum to react to stimuli efficiently. Furthermore it gives it the ability to behave as an active viscoelastic material, adapting its local mechanical parameters to also alter its contraction cycle ${ }^{25,26}$.

The contraction cycle itself is a prime subject of Physarum polycephalum research. There are multiple oscillation modes, for example the slime mold periodically ceases to oscillate during mitosis $(8 \mathrm{~h})$, and it also has a $20 \mathrm{~min}$ and a 60 min contraction cycle ${ }^{17}$. The most studied rhythm, however, is the radial tube contraction with a period of $60 \mathrm{~s}$ to $180 \mathrm{~s}^{27,28}$. The oscillation is highly susceptible to environmental factors: from lower oxygen ${ }^{29}$, light (see below), temperature ${ }^{30}$, mechanical perturbation (see below) or chemical stimuli such as inhibitors ${ }^{27}$, activators ${ }^{23}$, salts, glucose or nutrient me- 

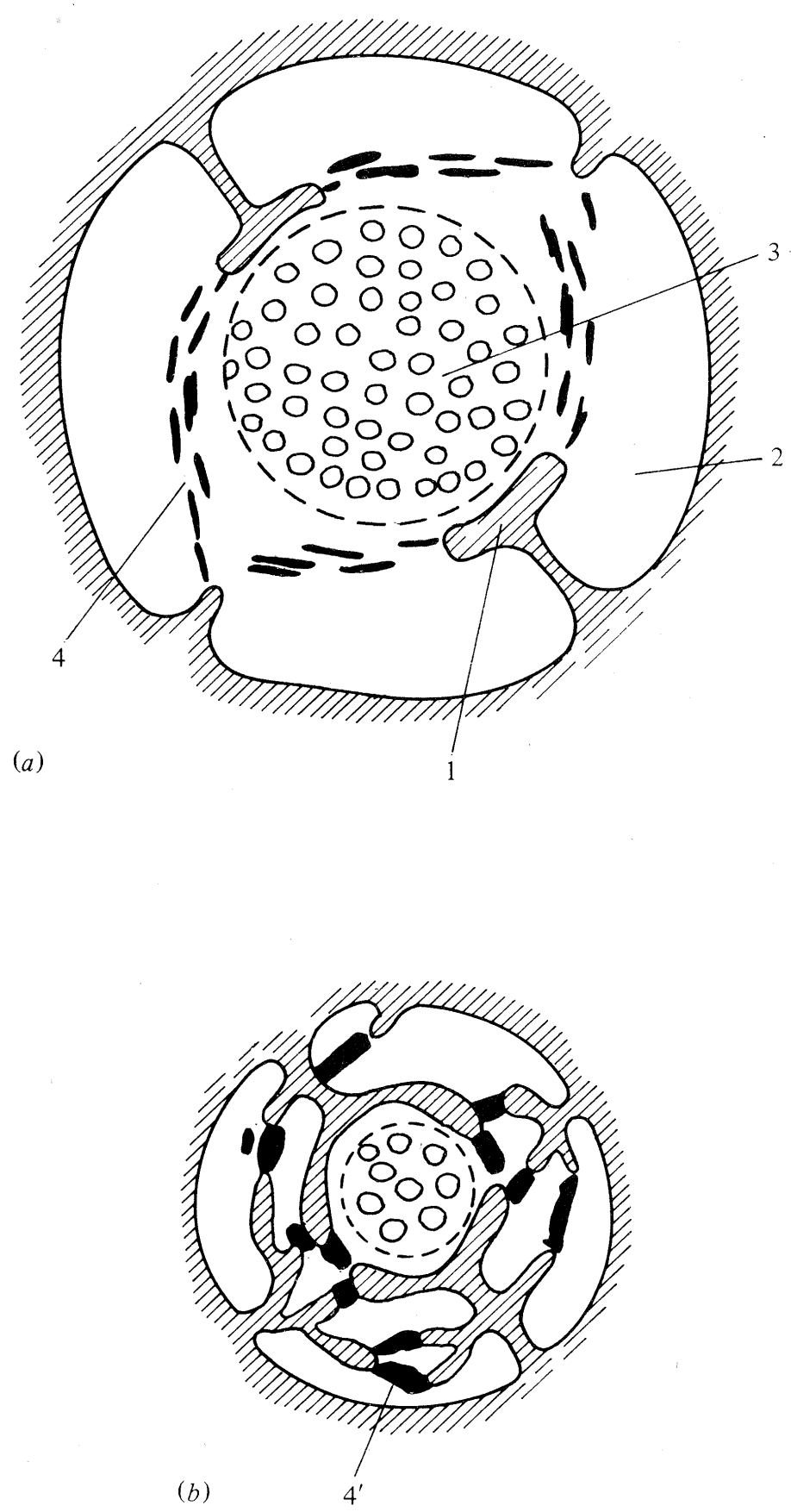

Figure 3: Schematic view of the ectoplasm contractions in a plasmodial tube cross section. (a) Relaxed cross section with plasmalemma invaginations (1), ectoplasm (2) and endoplasm (3). Actin filament bundles (4), attached to invaginations, contract rhythmically (4') thereby compressing tube cross section (b) and pushing mass elsewhere. Figure from 'Developmental Biology of Physarum' by Helmut W. Sauer, reproduced with permission of The Licensor through PLSclear. 
dia in general ${ }^{31}$. These influences cause the oscillation to adapt, changes which are best described with parameters for oscillatory motion: period $T$, phase $\varphi$, amplitude $A$ and wave length $\lambda$. In this way the radial contraction of a tube segment can be approximated by

$$
H(x, t)=H_{0}+A \cos \left(\frac{2 \pi x}{\lambda}-\frac{2 \pi t}{T}+\varphi\right),
$$

with $H_{0}$ the base radius, $x$ space and $t$ time. Note that contraction oscillations in Physarum polycephalum can also show overtone patterns ${ }^{32}$. Overtones have integer multiples of the dominant frequency most often with decreasing amplitude and the set of harmonics, i.e. dominant wave and overtones, can be expressed as

$$
H_{i}(x, t)=H_{0}+\sum_{i=1}^{N} A_{i} \cos \left(\frac{2 \pi i x}{\lambda}-\frac{2 \pi i t}{T}+\varphi_{i}\right),
$$

where $\lambda$ and $T$ are the wave length and period of the dominant wave respectively.

Each tube is interconnected to every other tube through the fluid flow and they are therefore constantly interacting, creating mesmerizing spatial patterns ${ }^{28}$. But more importantly the correlated patterns allow Physarum polycephalum to keep track of its own size by minimizing the difference in phase of contractions of neighbouring tubes. The resulting overall pattern is a single peristaltic wave of contractions running over the whole body span. This is regardless of the size of the network: even when cut into smaller networks each will start to employ a single peristaltic wave for its size. Besides measuring its own size this maximizes the particle distribution within the network through Taylor-dispersion ${ }^{33}$, an elegant way to distribute information and nutrients all the while being able to react collectively to external stimuli.

While the fluid flow connects each locally oscillating tube segment the flow is itself caused by tube contractions which leads to a back and forth flow in the network named shuttle streaming 34,35 . Disentangling the fluid flow from the contractions is a challenging task as they are inherently coupled. Nevertheless, Kamiya in 1940 found a beautiful way to at least separate them in a steady state: he invented the double-chamber method where one Physarum polycephalum specimen is separated into two pressure controlled chambers. Two networks are connected through a single tube which exhibits shuttle streaming. In each chamber tensiometers record the pressure differences which arise from the contractions. Furthermore 
the pressure in each chamber could be controlled to stall flow completely. Interestingly when stalling flows the applied pressure continued to oscillate showing that the contractions are inherent and a precursor to the fluid flow.

Arguably the more interesting phenomenon is the coupling between the fluid flow and the wall contraction. For example the peristaltic contraction wave over a specimen also leads to a coordinated flow pattern through peristalsis ${ }^{28}$. It collectively directs migration toward the leading edge or where environmental conditions are more favourable. When encountering attractive stimuli they release signaling molecules which are advected with the flow ${ }^{31}$. Yet the signaling molecule also leads to an expansion of the tubes which in turn pools mass from elsewhere to the nutrient source. The contractions elsewhere have to compete with the new pressure sink realigning their phase patterns. Additionally, the signaling molecule causes a local increase in contraction amplitude which in turn increases dispersion speed ${ }^{33}$. Together a feedback loop generates an adaptation strategy in an extended network where hydrodynamics coupled to local contractions rules create a global behaviour. Another example that shows the coupling of fluid flow and contraction shows that Physarum polycephalum tubes in pruning networks follow Murrays law ${ }^{36}$. Here shear forces of the fluid flow shape the diameter distribution of 3-connected nodes with a defined ratio between two smaller and one larger tube.

Consequently theoretical models about the self-organization of the tube oscillations coupled with hydrodynamics are a prime matter of research on Physarum polycephalum. The motivating questions answered range from 'How do oscillatory flows scale with size?'37, 'How are stresses localized to create traction?'38 or 'How does the plethora of contraction patterns come into existence?' 39,40 just to name a few. An overview of the models that arose from or were applied to Physarum polycephalum would go beyond the scope of this thesis but for further reading I recommend the following literature ${ }^{11,41}$. Most of these models are non-linear but still have physiologically relevant parameter ranges where stable patterns or comparable dynamics occur - a hotbed for new discoveries. In the present work the peristalsis theoretical framework is applied to discuss the efficiency of mass transport in Physarum polycephalum and is described in more detail in Section 2.3. 


\subsection{Phototaxis and photomorphogenesis of Physarum}

Slime molds need to be acutely aware of the light conditions of their surroundings. Their natural habitat is not underground and being a single cell does not allow for a skin as protection. Without a sophisticated cell exterior drying out or cell damage by UV light are constant threats to their well-being. At the same time when sporulating a higher vantage point, which are usually indicated by a higher light exposure, allows for a wider distribution of newly formed spores. So while the fragility is a high price to pay, the slime mold can use its gained benefit of fast adaptability to react appropriately to a variety of light conditions.

Light stimulated reactions of slime molds has been subject to research since 1876 and since then a wide range of reactions have been recorded 9 . In general they can be classified into two categories. Firstly phototaxis: an immediate relocation of body mass either towards light or away from it. And secondly photomorphogenesis: long-term and most often non-reversible changes in the morphology which incorporate some level of cellular differentiation. From a behaviour and biological standpoint it is useful to divide the reactions into these categories, but so far it is not clear if they share one or more pathways with different characteristics at different time points.

In my experiments I focus on light stimuli as a means to induce phototaxis and its concomitant mass transport. While mechanical stimuli were also used, light stimuli are easier to apply instantaneously to a defined area, can be quantified readily in terms of exposure and have a long history to rely on in Physarum polycephalum research. While phototaxis is the immediate reaction invoked and studied here, the insights from photomorphogenesis help to understand the molecular interplay that leads to behaviour. Here I am going to present the relevant studies done in the past century.

\subsubsection{Photomorphogenesis}

The first systematic approach to the influence of light on myxomycetes was done by William D. Gray in $1938^{42}$. The work was focused mostly on Physarum polycephalum but at the time the main point of interest was developing reliable culturing methods for myxomycetes in general. Cultures sporulated quickly in the lab which gave little to no time for controlled and quantitative experiments. Gray established that light is responsible for the sporulation process. Furthermore he found that the wavelength of the il- 
lumination was crucial for sporulation to occur. In his experiments only white and blue light on the order of $400 \mathrm{~nm}$ to $500 \mathrm{~nm}$ caused sporulation, where the wattage of the used light bulbs set a defined fruiting time. With alternating dark and light phases imitating a circadian or a $3 \mathrm{~s}$ rhythm he found that slime molds integrate the total light exposure, albeit in a nonlinear fashion. With his work he laid the foundation that light quality has a defining and complex interaction with slime molds.

Sporulation is a tunable all-or-nothing event 43,44 . The process is guided by a phytochrome system which are known in plants, bacteria and fungi to initiate differentiation. Blue light $(430 \mathrm{~nm}$ to $500 \mathrm{~nm})$ and far-red light ( $700 \mathrm{~nm}$ to $790 \mathrm{~nm}, \mathrm{FR})$ induces a state which after a given period of time and starvation irreversibly cause sporulation. When red light $(600 \mathrm{~nm}$ to $700 \mathrm{~nm}, \mathrm{R}$ ) is applied before the process is irreversible the state is 'turned off' and Physarum polycephalum does not sporulate. Consecutive irradiation of alternating $\mathrm{R}$ or FR pulses always lead to sporulation when ended with FR and stay as plasmodia when ended with $\mathrm{R}$.

Interestingly the FR induction of sporulation and the blue light induction differ. Besides FR and blue light also a heat shock can induce sporulation, but they all share the need for a starved plasmodium. On fed Physarum polycephalum plasmodia the blue stimulus is stored in the plasmodia and when starved will cause sporulation even when the starvation signal occurs a day later. The switchable FR signal on the other hand is transient and the probability of causing sporulation is close to zero when the starvation signal is given five hours after illumination.

The signaling cascades from a photon hitting Physarum polycephalum to a differentiation into another morphogenetic form are still poorly understood. The expression levels of the cytoskeletal proteins actin, $\alpha-$ and $\beta-$ tubulin adapt in response to light to evoke a structural change ${ }^{45}$, but which mechanism changes the expression pattern is not known. The recently published genome reveals promising gene locations with domains which have comparable functions in other species ${ }^{13}$. For example among the discovered photoreceptors are cryptochromes which are known for their blue-light reaction and are usually linked to expression patterns in a variety of bacteria and eukaryotes ${ }^{46,47}$. An additional interesting candidate for an unconventional photoreceptor is the LovA predicted protein. It combines PAS_9, a chromophore binding domain, with a RasGAP, a PB1 and a SAM domain. The latter domains are widespread and commonly associated with proteinprotein interactions or transducing signals and might thereby help to inte- 
grate, modulate and/or relay multiple signals. Even though the discovery of such genes only adds to the mystery of photomorphogenesis and phototaxis in slime molds it is fascinating to imagine the possible signaling pathways.

\subsubsection{Phototaxis}

Phototaxis is an active migration towards or away from light stimuli. Everyday examples are moths swarming around a light source showing positive phototaxis $4^{8}$ or cockroaches fleeing a room when light is turned on showing negative phototaxis ${ }^{49}$. While more complex organisms may include some decision making process ${ }^{48}$, prokaryotes on the simple side of the spectrum most often use a biased random walk strategy with a known pathway ${ }^{50}$. Arguably the most fascinating complexity resides somewhere in between, where mechanistic reactions are coupled in a non-linear fashion, e.g. by cell-cell interactions or fluid dynamics. That constitutes a research niche where phototaxis gains a lot of traction recently, e.g. for biofilms or for collective behaviour ${ }^{50}$. To give an example, the alga Volvox carteri achieves a coordinated phototactic response by an adaptive flagellar beat ${ }^{51}$. The colony grows on a spheric extracellular matrix and swims collectively towards light. Each cell reacts with an autonomous flagellar beat, but the frequency and polarity of the response is tightly coupled to the colony's sphere shape. This is a neat example how local rules cause a global behaviour. Furthermore, a set of such local rules can give rise to a decision-making process spanning the gap between mechanism and 'intelligence'.

In a similar manner slime molds show a collective phototactic response. Locally illuminated areas can be seen as individuals acting in an interconnected tissue. While slime molds mostly avoid light, the actual response depends on the wavelength, intensity and starvation level and can even lead to photoattraction. In either case mass is actively pumped through the network towards or away from the exposed areas. Pumping is a direct result of the contraction pattern therefore recent studies on phototaxis in Physarum polycephalum focused on the changes in the contraction patterns. I here want to give an overview of phototaxis in slime molds and the influence of light on the contraction patterns specifically. This is the basis for designing experiments and data analysis routines, as well as discussing 
the tactic response in accordance with the fluid flow.

The major phototactic response is photoavoidance. But why avoid light in the first place? Physarum polycephalum shies away from light when it is well-fed as it hampers growth. The dry weight of plasmodia increases more rapidly in the dark in comparison to illuminated specimen ${ }^{52}$. At the same time light strongly inhibits the glucose metabolism which might be the reason for a decreased growth rate ${ }^{53}$. The same is true for respiration, just $15 \mathrm{~s}$ after light exposure the oxygen uptake is strongly inhibited. Furthermore the reaction spectra of photoavoidance coincide with the action spectra for superoxide formation which act as radicals being highly toxic ${ }^{54}$. It is evident that $P$. polycephalum does not prosper in light hence the photoavoidance reaction. One has to make sure that illumination during culturing is kept at a bare minimum. On a side note the yellow pigments of P. polycephalum become bleached over time which is a useful indicator for light exposure in the lab.

Phototaxis is temperature, light intensity, wavelength and age dependent, where age is the time of starvation $44,55-57$. Young plasmodia shy away from white light when half of the specimen is illuminated and migrate towards the dark side ${ }^{58}$. Yet, with increasing age the speed of migration slows down and migration direction reverses towards the light $12 \mathrm{~d}$ to $13 \mathrm{~d}$ after starvation, albeit the migration is slowed down considerably at that point. This coincides with a decision for sporulation. Consequently for a controlled and fast tactic response Physarum polycephalum should be kept well-fed until the start of experiments.

For the light stimuli recognition Physarum polycephalum likely relies on photoreceptors, even though direct proof is still missing. The complex reaction makes isolation of those difficult, nevertheless at least four different photoreceptors were distinguished by action spectrometry 44,59 . Here action spectrometry gives the behavior response of the slime mold, i.e. the action of moving towards of away from a light source, for given a light stimulus with varying wavelengths and intensities. The genome furthermore revealed five photoreceptors of three different classes, which suggests in comparison to the action spectrometry that two of those have overlapping action spectra ${ }^{13}$. The spectrum has distinct peaks at $260 \mathrm{~nm}, 370 \mathrm{~nm}, 470 \mathrm{~nm}$ and $750 \mathrm{~nm}$ where the action was measured as the minimal light exposure required to evoke a minimal response. In detail the rate of mass reduction measured by the transmission brightness was recorded after light onset for 
various light intensities and extrapolated to the point of minimal response. Even with unknown photoreceptor kinetics such a response curve is congruent to the total absorption spectrum of four individual photoreceptors (or five where two have overlapping spectra). In line with the aging experiments, the sensitivity in the near UV range ( $300 \mathrm{~nm}$ to $400 \mathrm{~nm}$ ) decreases 15-fold upon starvation ${ }^{60}$, which points to the $370 \mathrm{~nm}$ photoreceptor as a main actor for negative phototaxis. Interestingly an increase of the temperature from $25^{\circ} \mathrm{C}$ to $31^{\circ} \mathrm{C}$ reduces the sensitivity to blue light (roughly $400 \mathrm{~nm}$ to $500 \mathrm{~nm}$ ), while the sensitivity to UV light stays the same. But the question remains how the photoreceptor which is responsible for the phototactic behaviour actually causes the movement of the slime mold away from the blue light. To answer this question we have to turn towards the tubes contraction patterns which connect the molecular action, i.e. the actomyosin mechanism, to a global pumping behaviour.

K.E.Wohlfarth-Bottermann was among the first to study the impact of light on the oscillation patterns systematically in Physarum polycephalum $^{27,30,61,62}$. In general, he focused on analyzing measurements as oscillations in time for Physarum polycephalum, an approach that laid the foundation for a signal processing style analysis on slime molds in general. At the time, tensiometric measurements were most commonly used which measured radial forces by long single strands ( $\mathrm{cm}$ scale $)^{63}$.

Wohlfarth-Bottermann and Block found that the frequency of the dominant oscillation mode drops upon blue light illumination ${ }^{61}$. They compared three different filters: $496 \mathrm{~nm}, 650 \mathrm{~nm}$ and $720 \mathrm{~nm}$, each with windows of $\sim 50 \mathrm{~nm}$. For the blue filter $(496 \mathrm{~nm}$, illuminance $=10000 \mathrm{~lx})$ and white light the frequency of oscillation drops from $\sim 10 \mathrm{mHz}$ to $6 \mathrm{mHz}$ roughly $20 \mathrm{~min}$ after the stimulus hits, with the decrease in frequency from $t=0 \mathrm{~min}$ to $\mathrm{t}=20 \mathrm{~min}$ being linear. Finally the frequency recovers after $20 \mathrm{~min}$ to $30 \mathrm{~min}$ to comparable values, with red light showing no measurable effect. The authors later also tried to pinpoint the means of signal transduction in the shuttle flow ${ }^{62}$. The frequency drop was delayed or did not occur at all when the flow was pointed away from the point of measurement through the illuminated area.

But besides these local characterisation of the contraction behaviour a global patterning and a coinciding change in the fluid transport mechanism in Physarum polycephalumnetworks is still missing. It seems evident that a local change in the frequency of the contractions as already recorded does change the pumping efficiency of the slime mold. Furthermore the 
amplitude and the spatial profile of the waves are likely key components for the pumping efficiency. We already know that the predominant spatial profile is a peristaltic wave across the full body span [28], hence we can turn to peristaltic pumping theory to investigate a changing contraction pattern on the pumping efficieny. In this way we can use a fast and reproducible blue light stimulus to induce a putative change in the actomyosin action, study the coinciding changes in the contraction patterns and correlate it to the resulting behaviour. A chain linking bio molecular changes due to environmental conditions to a macroscopic behaviour with the use of fluid dynamics without any central organisation unit relaying the information. In conclusion the question that arises at this point is: How does Physarum polycephalumchange its contraction patterns upon a light stimulus and how does it influence the pumping efficiency as a mean to induce mass transport?

\subsection{Peristaltic pumping theory}

Peristaltic pumping is a phenomenon most commonly known in the esophagus when swallowing or for transport in the intestines ${ }^{64}$. Characteristically it utilizes a moving local occlusion of a tube which pushes its contents forward creating net transport. An everyday example is a water-filled garden hose connecting two water reservoirs which is squeezed in a sliding motion towards one end. Even though the hose has the same shape before and after a net amount of water was pushed towards one reservoir. As Physarum polycephalum incorporates all the necessary components for peristaltic pumping it was proposed early on as a mass transport method ${ }^{65}$. Yet, the network body plan and dynamic phase relations, exhibiting a variety of standing and traveling waves, made it difficult to resolve a peristaltic wave in slime molds and was only done for a full network recently by Alim et al. ${ }^{28}$. Others have discussed the mixing of cytosol in microplasmodia tadpoles ${ }^{66}$ or their peristaltic waves ${ }^{67}$, but peristalsis as a pumping method for a net transport was left for investigation until now. To present the basis the following theoretical work builds on, I here want to present the theoretical framework of peristalsis mostly building on research done by Li and Brasseur ${ }^{68}$ and pioneered by Shapiro et al. ${ }^{69}$. 
First the governing equations are introduced. In particular the NavierStokes equation is the foundation to describe the motion of fluids in general,

$$
\rho\left(\frac{\partial \mathbf{u}}{\partial t}+\mathbf{u} \cdot \nabla \mathbf{u}\right)=-\nabla \bar{p}+\mu \nabla^{2} \mathbf{u}+\frac{1}{3} \mu \nabla(\nabla \cdot \mathbf{u})+\rho \mathbf{g},
$$

with $\rho$ the fluid density, $\mathbf{u}$ the flow velocity, $\bar{p}$ the hydrostatic pressure which is going to be pressure $p$ as the volume viscosity does not play a role ,$- \mu$ the dynamic viscosity and $\mathbf{g}$ the body accelerations. Furthermore, we apply the following approximations to the equation:

- Incompressibility: Physarum polycephalum is $90 \%$ water ${ }^{70}$ which is incompressible. Further, it was shown previously for cytoplasmic streaming in mammalian cells - which have less water content - that they can be seen as incompressible ${ }^{71}$. Therefore fluids in slime molds can be assumed as incompressible $(\nabla \cdot \mathbf{u})=0$ :

$$
\frac{\partial \mathbf{u}}{\partial t}+(\mathbf{u} \cdot \nabla) \mathbf{u}-\frac{\mu}{\rho_{0}} \nabla^{2} \mathbf{u}=-\frac{1}{\rho_{0}} \nabla p+\mathbf{g} .
$$

- Low Reynolds number: The Reynolds number $R e=2 U R / v<1$ is low given the flow velocities ${ }^{20}$ and kinematic viscosity in Physarum polycephalum $^{72}$. The resulting Stokes flow, named after Gabriel Stokes, describes a type of fluid flow where advective inertial forces cane be considered small compared with viscous forces (inertia $\frac{\partial u}{\partial t}$ and convection $(\mathbf{u} \cdot \nabla) \mathbf{u}$ are much smaller than diffusion $\frac{\mu}{\rho_{0}} \nabla^{2} \mathbf{u}$ and internal stress $\left.\frac{1}{\rho_{0}} \nabla p\right)$. Accordingly the Stokes equation is:

$$
-\mu \nabla^{2} \mathbf{u}=-\nabla p+\rho_{0} \mathbf{g} .
$$

- Force free: The forces which are acting on the liquid are introduced by the boundary conditions - by changing the volume to be filled. There are no other forces acting directly on the liquid $\left(\rho_{0} \mathbf{g}=0\right)$ :

$$
\mu \nabla^{2} \mathbf{u}=\nabla p .
$$

- Lubrication theory: From here on we consider a long slender tube with rotational symmetry. A tube in Physarum polycephalum is generally significantly longer than its thickness. The length scale disparity, i.e. substrate area vs. substrate thickness, results in constricted pressure gradients for the Stokes equation. In detail the pressure is uniform on 
each tube cross-section. In cylindrical coordinates the Stokes equation follows:

$$
\frac{\partial p}{\partial x}=\frac{\mu}{r} \frac{\partial}{\partial r}\left(r \frac{\partial u}{\partial r}\right)
$$

where $u$ the velocity component along the length axis $x$ and $v$ the velocity component in radial direction $r$. There is no flow in azimuthal direction due to symmetry.

- Mass conservation: As the fluid is incompressible and the system is continuous the total volume has to stay constant. At boundaries the total putative in- and out-flow have to even out

$$
\frac{1}{r} \frac{\partial(r v)}{\partial r}+\frac{\partial u}{\partial x}=0
$$

\subsubsection{Geometry and boundary conditions}

The symmetric tube elongates in the $x$ direction and the tube wall has a radial shape given by $H(x, t)$. The changes in shape $H(x, t)$ over time $t$ and space $x$ can be assumed to be periodic (train waves) or single waves traveling over the tube. Here, we consider only the first, but it should be mentioned that the results differ only by a constant factor.

For a peristaltic wave we can introduce the appropriate normalizations to the wavelength $\lambda$, the tube length $L$, a dominant radial scale $H_{0}$ and a wave speed $c$ to

$$
x=\frac{\hat{x}}{\lambda}, \quad r=\frac{\hat{r}}{H_{0}}, \quad t=\frac{c \hat{t}}{\lambda}, \quad u=\frac{\hat{u}}{c}, \quad v=\frac{\hat{v}}{k c}, \quad v=\frac{\hat{v}}{k c}, \quad p=\frac{\hat{p} H_{0}^{2}}{\mu c \lambda},
$$

to non-dimensionalize the problem, with $k=H_{0} / \lambda$ the wave number.

We consider the 'no-slip', the 'no-penetration' and axial symmetry boundary conditions. I.e. the liquid has negligible velocity, besides the wall movement, at the wall, no liquid can pass through the tube walls and there is no radial flow through the tube center

$$
\left.u\right|_{r=H}=0,\left.\quad v\right|_{r=H}=\frac{\partial H}{\partial t},\left.\quad \frac{\partial u}{\partial r}\right|_{r=0}=0,\left.\quad v\right|_{r=0}=0 .
$$


From Eq. (3) considering the boundary conditions follows

$$
\begin{aligned}
r \frac{\partial u}{\partial r} & =\int r \frac{\partial p}{\partial x} \mathrm{~d} r=\frac{r^{2}}{2} \frac{\partial p}{\partial x}+C_{1}, \quad \text { with }\left.\frac{\partial U}{\partial r}\right|_{r=0}=0 \Rightarrow C_{1}=0 ; \\
u & =\frac{1}{2} \frac{\partial p}{\partial x} \int r \mathrm{~d} r=\frac{r^{2}}{4} \frac{\partial p}{\partial x}+C_{2}, \quad \text { with }\left.u\right|_{r=H}=0 \Rightarrow C_{2}=-\frac{1}{4} H^{2} \frac{\partial p}{\partial x} \\
& \Rightarrow u=\frac{1}{4} \frac{\partial p}{\partial x}\left(r^{2}-H^{2}\right) .
\end{aligned}
$$

And using continuity Eq. (4) to get the radial velocity component

$$
\begin{aligned}
0 & =\frac{1}{r} \frac{\partial(r v)}{\partial r}+\frac{1}{4} \frac{\partial^{2} p}{\partial x^{2}}\left(r^{2}-H^{2}\right)-\frac{1}{2} \frac{\partial p}{\partial x} H \frac{\partial H}{\partial x} \\
r v & =\frac{1}{2} \int\left[r \frac{\partial p}{\partial x} H \frac{\partial H}{\partial x}-\frac{1}{2} r \frac{\partial^{2} p}{\partial x^{2}}\left(r^{2}-H^{2}\right)\right] \mathrm{d} r \\
v & =\frac{r}{4} \frac{\partial p}{\partial x} H \frac{\partial H}{\partial x}-\frac{1}{4} \frac{\partial^{2} p}{\partial x^{2}}\left(\frac{r^{3}}{4}-\frac{r H^{2}}{2}\right)+C_{3}, \quad \text { with }\left.v\right|_{r=0}=0 \Rightarrow C_{3}=0 \\
& \Rightarrow v=\frac{r}{4}\left(\frac{\partial p}{\partial x} H \frac{\partial H}{\partial x}-\frac{\partial^{2} p}{\partial x^{2}}\left(\frac{r^{2}}{4}-\frac{H^{2}}{2}\right)\right) .
\end{aligned}
$$

We want to formulate the pressure gradient arising from the wall movement. For that we can use the radial velocity Eq. (8) and evaluate it at the wall boundary

$$
\left.v\right|_{r=H}=\frac{\partial H}{\partial t}=\frac{H}{4}\left(\frac{\partial p}{\partial x} H \frac{\partial H}{\partial x}+\frac{H^{2}}{4} \frac{\partial^{2} p}{\partial x^{2}}\right)=\frac{1}{16} \frac{1}{H} \frac{\partial}{\partial x}\left(H^{4} \frac{\partial p}{\partial x}\right) .
$$

To get the pressure gradient building up from each tube segment along the whole tube, we can integrate Eq. (9) along the tube

$$
\begin{aligned}
16 \int_{0}^{L} H & \frac{\partial H}{\partial t} \mathrm{~d} x=H^{4} \frac{\partial p}{\partial x}+C_{4}(t) \\
\Rightarrow & \frac{\partial p}{\partial x}=\frac{1}{H^{4}}\left(C_{4}(t)+16 \int_{0}^{x} H(s, t) \frac{\partial H(s, t)}{\partial t} \mathrm{~d} s\right) .
\end{aligned}
$$

At each end of the tube along the symmetry axis we have an putative flow in- and out-let. Instead of defining the velocities we can also define the flow fully by setting pressure boundary conditions for the inlet $p_{0}(t)$ and for the outlet $p_{L}(t)$. The total pressure is then given by

$$
p(x, t)=p_{0}(t)+\int_{0}^{L} \frac{\partial p}{\partial x} \mathrm{~d} x, \quad \text { with }\left.p\right|_{x=0}=p_{0}(t) \quad \text { and }\left.\quad p\right|_{x=L}=p_{L}(t) .
$$

Combining Eq. (10) and Eq. (11) we find that the constant $C_{4}$ to be

$$
C_{4}(t)=\frac{\Delta p(t)-16 \int_{0}^{L} H^{-4}\left(s_{1}, t\right)\left(\int_{0}^{s_{1}} H\left(s_{2}, t\right) \frac{\partial H\left(s_{2}, t\right)}{\partial t} \mathrm{~d} s_{2}\right) \mathrm{d} s_{1}}{\int_{0}^{L} H^{-4}(s, t) \mathrm{d} s} .
$$


The flow rate through a tube is given by

$$
\hat{Q}(\hat{x}, \hat{t})=\iint_{\hat{A}} \hat{\mathrm{v}} \mathrm{d} \hat{A}=2 \pi \int_{0}^{\hat{H}} \hat{u} \hat{r} \mathrm{~d} \hat{r} \text {. }
$$

We want to normalize the flow rate. in particular we normalize the flow rate to the flow rate of a completely occluded pump averaged over one wave period $\pi H_{0}^{2} c$ to

$$
Q(x, t)=\hat{Q} /\left(\pi H_{0}^{2} c\right)=\frac{1}{8} \frac{\partial p}{\partial x} H^{4} .
$$

Fortunately we know $\frac{\partial p}{\partial x}$ by now (see Eq. (10) with Eq. (12)) and can fully express the flow

$$
Q(x, t)=\frac{2 \int_{0}^{L} H^{-4}\left(s_{1}, t\right)\left(\int_{x}^{s_{1}} H\left(s_{2}, t\right) \frac{\partial H\left(s_{2}, t\right)}{\partial t} \mathrm{~d} s_{2}\right) \mathrm{d} s_{1}-\frac{\Delta p(t)}{8}}{\int_{0}^{L} H^{-4}(s, t) \mathrm{d} s} .
$$

Now Brasseur et al. furthermore assumed the pressure difference at the end of the tubes to be constant, i.e. $\Delta p=$ const.. That is to characterize the pumping performance with the time-averaged volume flow rate

$$
\bar{Q}=\frac{1}{T} \int_{t_{0}}^{t_{0}+T} Q(x, t) \mathrm{d} t \text {, with } T=1 \text { for train waves, }
$$

as the following

$$
\begin{gathered}
\bar{Q}=\bar{Q}_{0}\left(1-\frac{\Delta p}{\Delta p_{0}}\right) \\
\bar{Q}_{0}=\frac{2}{T} \int_{0}^{T} \frac{\int_{0}^{L} H^{-4}\left(s_{1}, t\right)\left(\int_{x}^{s_{1}} H\left(s_{2}, t\right) \frac{\partial H\left(s_{2}, t\right)}{\partial t} \mathrm{~d} s_{2}\right) \mathrm{d} s_{1}}{\int_{0}^{L} H^{-4}(s, t) \mathrm{d} s} \mathrm{~d} t \\
\Delta p_{0}=\bar{Q}_{0}\left[\frac{1}{T} \int_{0}^{T} \frac{\mathrm{d} t}{\int_{0}^{L} H^{-4} \mathrm{~d} s}\right]^{-1} .
\end{gathered}
$$

Equations Eq. (17) to Eq. (19) can be used to compare the the pumping efficiency of different tube shapes $H(x, t)$. The non-linearities in the tube shape $H^{-4}$ and $H \frac{\partial H}{\partial t}$ make finding a solution for the integrals difficult even for easy train waves like the one shown in Eq. (1). For integral periodic train waves with only a dominant wave a solution can be found and was discussed by Shapiro et al., yet here we want to specifically compare the influence of overtones on the pumping efficiency. Hence we use numerical methods to find solutions to the problem. 


\subsection{Scope of the thesis}

Here I present my work on mass transport in Physarum polycephalum. Two principle experimental approaches are used, both of which cause a net mass transport within the network, and an in-depth quantitative analysis. A cutting stimulus was known from preliminary tests to invoke a regrowth of network at the cut site yet the coinciding contraction patterns were still unknown. The experimental methods and in particular the analysis routine were developed here to study contraction patterns in slime mold networks and revealed new insights in spatial and temporal patterns. We found the contractions to cease right after a cut stimulus and subsequently to restart vigorously into a coordinated contraction or 'pumping' phase. The end of the pumping phase coincides with the maximal fan growth at the cut site after which the slime mold decides to grow elsewhere. The work on wounding highlights the self-organization and the interplay of various regions within the network. A single tube's contraction is coordinated with respect to every other tube within its network and works towards reconnecting severed parts when cut.

At the same time the temporal patterns in contractions revealed periodic, but non-sinusoidal characteristics, especially so given a stimulus. For easier experimental handling, quantification and due to the extensive research to rely on for phototaxis and photomorphogenesis in Physarum polycephalum I switched to a negative phototactic blue light stimulus. The contraction frequencies, and in particular the overtones, adapted when Physarum polycephalum was subjected to a blue light stimulus. Theoretical work on the peristaltic pumping theory with varying contraction patterns revealed the phase relation between overtone and dominant frequency to be crucial. An adaption in the phase relation towards more optimal pumping was found to occur in Physarum polycephalum blue light stimuli experiments highlighting in detail the adaption proficiency of slime molds.

Before presenting the results the used materials, the culturing methods, the experimental setup and methods as well as the computational methods are explained in-depth. Lastly an outlook is given where this work, but also the whole research field might head towards in the foreseeable future. 
Part II

MATERIALS AND METHODS 

In the following the materials and methods for Physarum polycephalum culture and experiments are listed. They are given in protocol style for easy reuse. An extensive list of the ingredients with details and supplier information can be found in Section A.1.

\subsection{Media recipes}

M 1.1 SEMI-DEFINED MEDIUM (SDM)

$10 \mathrm{gl}^{-1} \quad \mathrm{D}(+)$-glucose anhydrous

$10 \mathrm{gl}^{-1}$ Difco-Bacto-Soytone

$1.5 \mathrm{gl}^{-1} \quad$ Yeast extract

$3.54 \mathrm{gl}^{-1} \quad$ Citric acid monohydrate

$2 \mathrm{gl}^{-1}$ Potassium dihydrogen phosphate

$0.6 \mathrm{gl}^{-1} \quad$ Calcium chloride dihydrate

$0.6 \mathrm{gl}^{-1}$ Magnesium sulfate heptahydrate

$0.224 \mathrm{gl}^{-1} \quad$ EDTA

$0.06 \mathrm{gl}^{-1}$ Iron(II) chloride tetrahydrate

Mix in double distilled water. Set $\mathrm{pH}$ to 4.6 with $\mathrm{KOH}$ pellets. Autoclave with a liquid setting for 20 minutes and store at room temperature. Prior to use add $10 \mathrm{ml}$ haematin solution ${ }^{\dagger}$ per liter and $250 \mu \mathrm{g} \mathrm{ml}^{-1}$ penicillin or streptomycin $\left(-20^{\circ} \mathrm{C}\right)$ after autoclaving.

M 1.2 BALANCED SALT SOLUTION (BSS)

$4.2 \mathrm{gl}^{-1}$ Dipotassium phosphate

$3 \mathrm{gl}^{-1}$ Citric acid monohydrate

$0.25 \mathrm{gl}^{-1}$ Sodium chloride

$0.21 \mathrm{gl}^{-1} \quad$ Magnesium sulfate heptahydrate

$0.05 \mathrm{gl}^{-1}$ Calcium chloride dihydrate

Dissolve in double distilled water. Set $\mathrm{pH}$ to 5.0 with $1 \mathrm{M} \mathrm{KOH}$ or $\mathrm{KOH}$ pellets. Autoclave with liquid setting for 20 minutes and store at room temperature. 


\section{1.3 LYSOGENY BROTH (LB) AND PLATES}

$10 \mathrm{gl}^{-1} \quad$ Bacto-tryptone

$5 \mathrm{gl}^{-1}$ Yeast extract

$10 \mathrm{gl}^{-1}$ Sodium chloride

Dissolve in double distilled water and set $\mathrm{pH}$ to 7.0 with $5 \mathrm{M} \mathrm{NaOH}$. $\mathrm{Au}-$ toclave with liquid setting for 30 minutes and store at room temperature. Optionally, for plates add $15 \mathrm{gl}^{-1}$ agar ( $\left.\equiv 1.5 \%\right)$ and pour into Petri dishes. Adapted from ${ }^{73}$.

M 1.4 HAEMATIN SOLUTION

Dissolve $0.05 \mathrm{~g} \mathrm{Hemin}^{\dagger}$ in $100 \mathrm{ml} 1 \% \mathrm{NaOH}^{\dagger}$ solution and autoclave it. After cooling down keep at $4{ }^{\circ} \mathrm{C}$ and in the dark.

M 1.5 FORMALIN KILLED BACTERIA BUFFER (FKBB)

$7 \mathrm{gl}^{-1}$ Disodium phosphate

$4 \mathrm{gl}^{-1}$ Sodium chloride

$3 \mathrm{gl}^{-1}$ Potassium dihydrogen phosphate

$0.1 \mathrm{gl}^{-1}$ Magnesium sulfate heptahydrate

Dissolve in double distilled water and autoclave with liquid setting for 20 minutes. Store at room temperature.

\section{1.6 FORMALIN KILLED BACTERIA STOCK}

\section{Procedure}

1. (Day 1)

Inoculate $2 \mathrm{ml}$ of FKBB with E.coli (Strand HB101) by picking a colony which grew on an LB plate. Leave for growth overnight.

2. (Day 2)

Inoculate $2 \times 250 \mathrm{ml} \mathrm{LB}$ medium in 11 Erlenmeyer flasks with $1 \mathrm{ml}$ overnight culture each. Incubate overnight in shaking culture at $37^{\circ} \mathrm{C}$ and $180 \mathrm{rpm}$.

3. (Day 3)

Shake flasks gently. Transfer suspension into $50 \mathrm{ml}$ conical centrifuge tubes and centrifuge at $4000 \mathrm{rpm}$ at $4{ }^{\circ} \mathrm{C}$ for $10 \mathrm{~min}$.

4. Re-suspend the pellets in $2.5 \mathrm{ml} \mathrm{FKBB}$ each (total of $25 \mathrm{ml}$ ). 
5. Transfer the suspension into a $100 \mathrm{ml}$ reagent bottle, add $4 \mathrm{ml}$ of $40 \%$ formaldehyde-solution LB medium and mix by gentle inversion.

6. Incubate overnight at $4{ }^{\circ} \mathrm{C}$.

7. (Day 4)

Transfer suspension into $50 \mathrm{ml}$ conical centrifuge tube and centrifuge at $4000 \mathrm{rpm}$ at $4^{\circ} \mathrm{C}$ for $10 \mathrm{~min}$.

8. Re-suspend in $25 \mathrm{ml}$ FKBB solution. For complete re-suspension shake tube and pipette suspension up and down.

9. Add $2.5 \mathrm{ml}$ of $1 \mathrm{M}$ glycerol (frozen) and incubate for $1 \mathrm{~h}$ at $37^{\circ} \mathrm{C}$ at $180 \mathrm{rpm}$.

10. Wash twice with $25 \mathrm{ml} \mathrm{FKBB}$ and centrifuge at $4000 \mathrm{rpm}$ at $4{ }^{\circ} \mathrm{C}$ for $10 \mathrm{~min}$. Interruption of protocol is possible here: store suspension overnight in the fridge optionally.

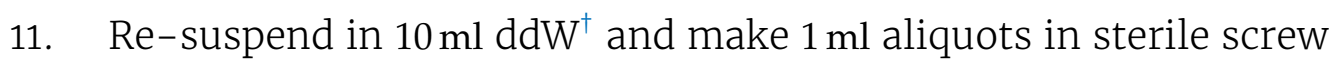
capped tubes and store at $4^{\circ} \mathrm{C}$.

Sterility test

Streak out sample $(20 \mu \mathrm{l})$ of the suspension on a LB plate and incubate at $37^{\circ} \mathrm{C}$ overnight. Nothing should grow.

\subsection{Physarum polycephalum culture}

Physarum polycephalum in laboratory setting is most often kept either in a microplasmodia culture ${ }^{74}$ or in an oat flake culture ${ }^{28}$. Microplasmodia culture is preferred as one can precisely control the nutritional state and amount of the slime mold. Furthermore, plasmodia grown this way are free from oat flake residues or food vacuoles, giving a cleaner sample for imaging. The Physarum polycephalum samples that were used here are ordered from Carolina as sclerotia. Generally all Physarum polycephalum samples should be kept in the dark for storage if not stated otherwise.

M 2.1 INITIAL MICROPLASMODIA CULTURE

\section{Timing}

Carry out every 2 to 3 months.

\section{Procedure}

Inoculate in sterile conditions. Keep incubator at $25^{\circ} \mathrm{C} / 180 \mathrm{rpm}$ 
1. Put Physarum sclerotia ${ }^{\dagger}$ cutout with the sclerotia side facing down on an agar plate ${ }^{\dagger}$.

2. Incubate for $2 \mathrm{~d}$ at $25^{\circ} \mathrm{C}$.

3. Cut out agar piece incl. plasmodium of about $2 \mathrm{~cm}$ to $3 \mathrm{~cm}$ in diamter.

4. Add SDM and BSS (1:1) to an empty Petri dish ${ }^{\dagger}$ and place the agar piece with Physarum plasmodium into the dish. Make sure the medium barely reaches plasmodium itself.

5. Incubate for $2 \mathrm{~d}$ at $25^{\circ} \mathrm{C}$. Network should spread into the media.

6. Discard the agar piece and transfer the media including plasmodium into an Erlenmeyer flask with $50 \mathrm{ml}$ SDM and $50 \mathrm{ml}$ BSS medium.

7. Continue with Section M 2.2.

M 2.2 INOCULATION OF MICROPLASMODIA

\section{Condition}

Microplasmodia in SDM/BSS suspension have to be yellow-orange ${ }^{74}$,

$0.5 \mathrm{~mm}$ to $3 \mathrm{~mm}$ in diameter, and have an irregular shape.

Timing

Carry out inoculation every $2 \mathrm{~d}$ to $3 \mathrm{~d}$.

\section{Procedure}

1. Add $50 \mathrm{ml}$ SDM to a $250 \mathrm{ml}$ Erlenmeyer flask with indented baffles.

2. Add $50 \mathrm{ml}$ BSS to the Erlenmeyer flask.

3. Gently shake culture flasks by hand. Transfer $1 \mathrm{ml}$ to $2 \mathrm{ml}$ of $\mathrm{mi}-$ croplasmodia culture to freshly prepared media. Make sure to use low pressure not to rupture the microplasmodia.

4. Transfer to incubated shaker $\left(25^{\circ} \mathrm{C} / 180 \mathrm{rpm}\right)$ and keep in the dark.

M 2.3 INITIAL OAT FLAKE CULTURE

See Section M 2.1, Steps 1. and 2..

M 2.4 INOCULATION OF OAT FLAKE CULTURE

Condition

Non-starved plasmodia should have dense network structure as well 
as thick connective tubes. Fans should be widely fanned (large angle). The whole network should be brightly yellow.

\section{Timing}

Carry out inoculation every $2 \mathrm{~d}$ to $3 \mathrm{~d}$.

\section{Procedure}

1. Place oat flake $e^{\dagger}$ onto agar plate ${ }^{\dagger}$ containing plasmodia and incubate for $2 \mathrm{~h}$ to $3 \mathrm{~h}$ if placed directly on plasmodia or overnight when left on agar.

2. Transfer freshly colonized oat flake onto new agar plate.

3. Add additional oat flakes depending on growth state. Add tap water on oat flakes to fixate and keep moisture.

4. Use wax film to seal the Petri dish and keep at $25^{\circ} \mathrm{C}$.

\subsection{Microscope setup and data acquisition}

The principle way of data acquisition in this work is movie recording with a transmitted light microscope. The microscope setup which was used is described in the following and is schematically represented in Figure 4. Furthermore the protocols for blue light stimulus experiments and the cutting experiments are explained.

Images were recorded using a Zeiss Axio Zoom v16 equipped with a Zeiss PlanNeoFluar 1x/0.25 objective and a Hamamatsu ORCA-Flash 4.0 digital camera. The zoom capabilities were necessary to adjust the field of view as the slime mold networks sizes vary between micrometer and centimeter sizes. The microscope setup is shielded from ambient light by a canopy to reduce enviromental stress on the specimen. Transmitted light is supplied with a combined trans-illumination motorized stage (Zeiss Transillumination top $450 \mathrm{mot}$ ) which supports a brightfield light source as well as motorized stage movement. If not mentioned otherwise, brightfield illumination was used. To reduce the photomorphogenetic and phototactic effect (see Section 2.2.2) on Physarum polycephalum the light intensity was kept at $30 \%$ or lower of the maximal transmission brightness as read from the SYCOP-3 control panel. The exposure time was set to $500 \mathrm{~ms}$ which still retains clear cell boundaries. Furthermore, a green filter foil was attached to the bottom of each imaged Petri dishes (139 Primary Green, LEE Filters, Transmission peak at $530 \mathrm{~nm}$, FHW $45 \mathrm{~nm}$ ). 
To stop drying out of agar and to keep a moist environment for Physarum polycephalum to grow an incubator (OKOlab) is used on the stage top including temperature control. The temperature was set to $25^{\circ} \mathrm{C}$ and the humidity was controlled with an airflow through demineralized water. We furthermore included an indium tin oxide top glass plate to keep condensation off the glass by heating it to $27^{\circ} \mathrm{C}$. For image acquisition and subsequent data handling Zeiss Zen 2.3 Pro software was used.

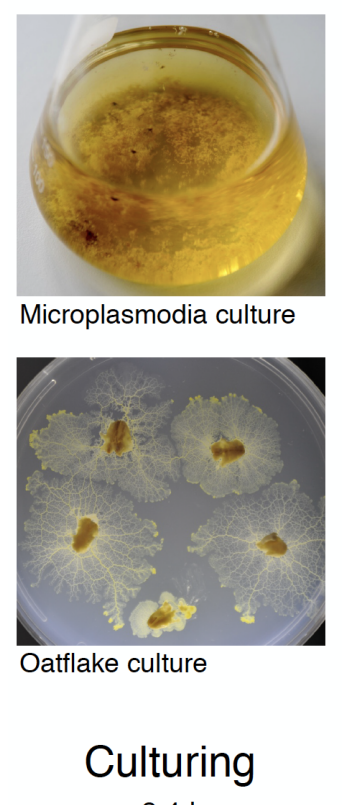

$2-4 d$

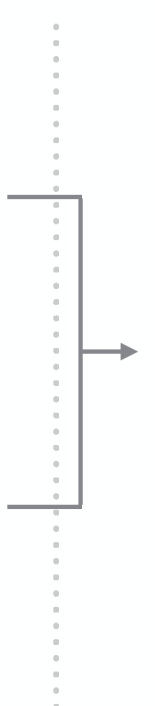

\section{Selection}

10-30min

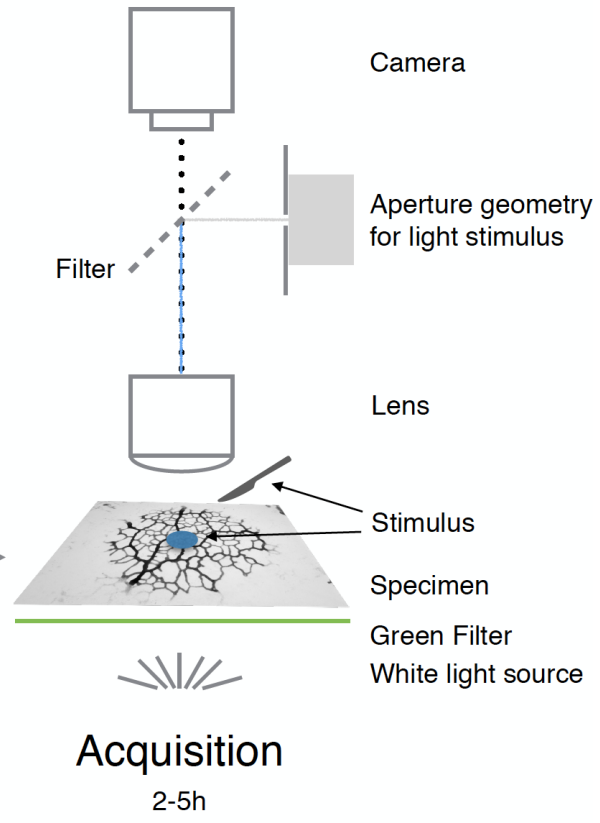

Figure 4: Schematic experiment setup. Firstly Petri dishes have to be prepared either from microplasmodia or oat flake culture. Secondly samples with sutiable networks are selected. Lastly the Petri dish is imaged with a Zeiss Axio Zoom v16 microscope and a Hamamatsu ORCA-Flash 4.0 digital camera. To block out ambient stimuli light a green filter (139 Primary Green, LEE Filters) is added above the light source. The aperture in the fluorescent pathway controls the spot size for a blue light stimulus with a Zeiss $38 \mathrm{HE}$ excitation BP 470/40 filter (Filter). Alternatively the blue light stimulus or a cutting stimulus can be applied and follows the same preliminary procedure.

\subsubsection{Blue light stimulation}

Plate $200 \mu \mathrm{l}$ dense microplasmodia into a petri dish centrally. Prepare multiple such Petri dishes $(\geq 10)$ for screening and selection (see Figure 4 for details). Leave overnight to grow in the dark. Cut networks to a size of 
roughly $30 \mathrm{~mm}^{2}$ with an inoculation loop $90 \mathrm{~min}$ before light exposure to mitigate any reaction from cutting 75 . Make sure that networks have low hierachy and are fairly isotropic. Adjust area to be illuminated with the fluorescent light aperture (Zeiss $38 \mathrm{HE}$, excitation BP $450 \mathrm{~nm}$ to $490 \mathrm{~nm}$ filter for blue light) to a size of roughly $30 \%$ to $50 \%$ of the total network size at the start of recording. If needed take an auto-fluorescence image of the agar by moving the focus plane $500 \mu \mathrm{m}$ into the agar and record one image without transmitted light for $3 \mathrm{~s}$. The imprint gives the exact stimulated area for comparison. Adjust transmitted light intensity to fill the dynamic range maximally with an exposure time of $500 \mathrm{~ms}$. Start image recording $60 \mathrm{~min}$ before fluorescent light exposure and acquire image every 3 to 6 seconds. Record until stimulated area is completely pruned. Depending on the size and the stimulated light intensity that takes up to $2 \mathrm{~h}$.

\subsubsection{Cutting plasmodial tube}

Plate $100 \mu \mathrm{l}$ to $200 \mu \mathrm{l}$ dense microplasmodia into a Petri dish centrally, but distributed in a patch roughly $3 \mathrm{~cm}$ in diamater. Prepare multiple such Petri dishes $(\geq 10)$ for screening and selection. Leave overnight to grow in the dark. Screen for small networks $\left(30 \mathrm{~mm}^{2}\right.$ ) preferably with a single connecting tube which, when severed, splits the network in two. Place plate into stage top incubator. Adjust transmitted light intensity to fill the dynamic range maximally with an exposure time of $500 \mathrm{~ms}$. Start image recording $60 \mathrm{~min}$ before cutting and acquire image every 3 to 6 seconds. Apply cut manually, using a microinjection needle with a blunt tip. The needle tip is held above the surface of the agar at a small angle and quickly dragged across the chosen plasmodial tube. Record for additional $2 \mathrm{~h}$ afterwards. 

Most of the findings in this work are based on the data analysis of bright field images, see Figure 5. In particular networks formed by slime molds can be approximated by axis-symmetric tubes and connecting nodes. In network topology terminology the tubes are considered as edges and the connecting nodes as vertices. In our application we refer to branches instead of edges, as the measured quantities of every point along the branch is essential and not only a single connection indication. In this representation every point along a branch may be attributed with information about the local tube cross section, e.g. the flow rate, flow velocity and crucially the respective radius. The time evolution of the radius is the local contraction pattern and can be seen as a signal encoding information about the slime molds current status. In the following I present my methods of obtaining a reasonable data representation of slime mold bright field experiments and how to I obtain quantities from this data. If not stated otherwise, all code was written in Matlab. Parts of the code and the initial concept were originally written and coded by Gabriel Amselem and Karen Alim, a basis to build up on I am very grateful for. I extended, added and rewrote the majority of the code in this project and in particular the analysis routines were written from scratch.

I want to point out that the following chapter doubles as a guideline for how to use the written code as well as a critical assessment of the used methods and/or parameters where applicable. Therefore parts may be written in an in-depth explanatory fashion while others rather present details of the code usage. Keeping the structure focused on the respective parts, i.e. network extraction, analysis and phase difference determination, helps to get a thorough understanding of the choices done while giving the reader the option to reproduce the obtained results.

\subsection{Code handling}

The code is written primarily in Matlab with parts of the cluster computing handling written in bash scripts and is available in source code. If not 


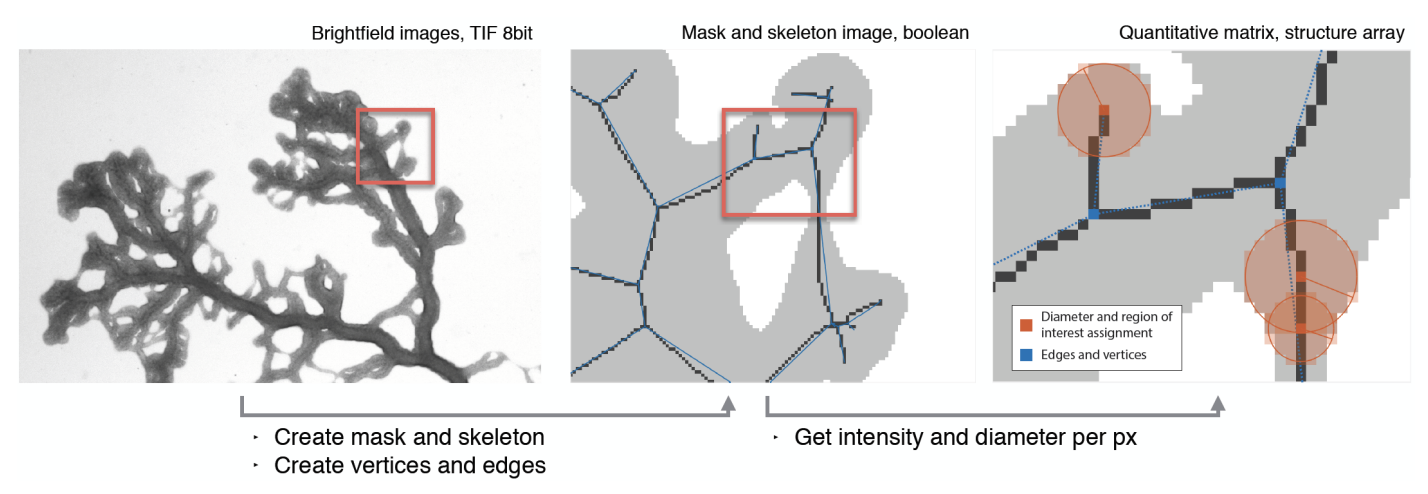

Figure 5: Schematic of the data analysis routine. Acquired brightfield images are binarized and subsequently skeletonized to get a mask and a skeleton of the Physarum polycephalum network. For each skeleton pixel associated quantitative parameters can be computed from the local intensity or diameter data.

handed out with this manuscript please contact the author for a copy of the code. Furthermore parts of the analysis was done with a kymograph based selective approach which is explained in the supplement A.4. The following focuses on the extraction of network-based quantities from the bright field images and the phase difference determination.

The code contains two major scripts meant to be run on a grid computing cluster and various scripts for offline use. The two major code scripts, the network analysis and the phase difference determination, have a similar structure: a compilation script, a run script and a submit writing script all written in bash. The former is further segmented into four subsets to be run subsequently for a full analysis of a dataset: variance calculation, network extraction, stitching data and analysis. Together with the phase difference analysis each of the five subsets is written in Matlab and has a primary file named driver [...] . mat which contains a similar structure and is the starting point for each job running online or offline.

The driver file contains a preamble where a parameter file, with a fixed name of parameters.txt, can be loaded by the compiled code. Therefore compilation is only necessary for new iterations of the code, otherwise an adaption to a new data set only requires a change of the parameters. Following the preamble is a listing of mandatory parameters and default values for necessary, but not mandatory parameters. No other parameters will be used by the program. The default values and effects of the script specific parameters will be explained in the respective sections and have a prefix of the respective job, i.e. NE for network extraction, ST for stitching and 
VA for variance calculation. The other parameters are used by every code and explained in the header of the parameters.txt file.

\subsection{Network extraction}

In general images recorded as a time series are processed as 8-bit uncompressed TIFs. At first every image is processed separately, then the results are stitched together and lastly the collective is analysed. In the first part the network extraction focuses on creating a binary mask from the bright field images, a subsequent skeletonization and lastly a measurement of the local intensity and diameter of the tube. I want to mention that, in order to remove artifacts in the masking of brightfield images, Noah Ziethen developed a variance mask function during his time as a Bachelor student in our group. I am very grateful for his work and want to here also briefly show the advantages of including a variance mask for the network extraction procedure.

The local intensity of brightfield images represents a good outline for the network formed by Physarum polycephalum, see Figure $6 \mathrm{~A}$, as the transmitted light is blocked by the slime mold itself. Yet due to cutting or when obstructing granules are present, i.e. oat flake residues, artifacts can occur where low intensities are not indicative of the specimen, see top right corner in Figure $6 \mathrm{~A}$. Here the variance over time outlines only the active slime mold more accurately and in particular shows the borders prominently due to the contractions giving a high variability in the cell wall, see Figure $6 \mathrm{~B}$. If the variance option is selected, VAprunOpt $=1$, the variance of the brightfield intensity is calculated for image subsets of a size defined by the parameter VAintervalVar. In particular the logarithm of the variance is used as the change of intensity within a tube can be small compared to the variance in the tube walls, yet is still comparatively high to the background noise. The image subset should be set to a size including at least one oscillation cycle to remove contraction artifacts from non-integral waves, i.e. not including the whole range. While the variance mask gives a better outline of the slime mold some internal parts - especially fan regions - are better masked with the intensity profile. Therefore a composite image is created by adding the variance and brightfield image normalized from 0 to 255 (8bit). Before combining the background is removed with a rolling- 


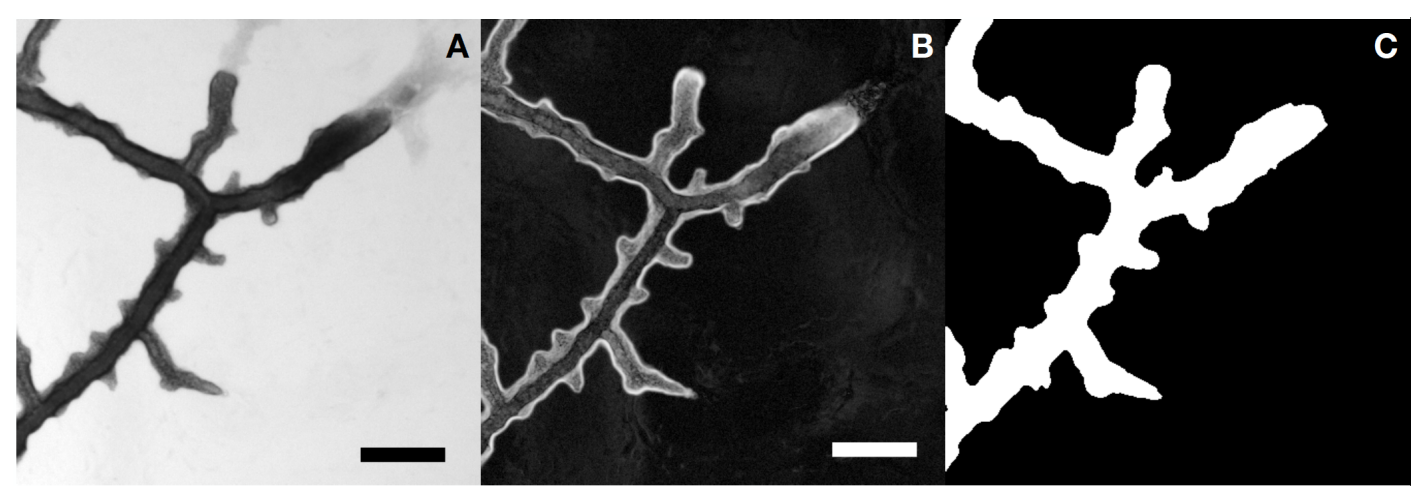

Figure 6: Comparison of the brightfield image, variance and resulting mask. (A) Exemplary brightfield image of Physarum polycephalumnetwork. Scale bar $=500 \mu \mathrm{m}$. (B) Logarithm of the variance of 51 images in time. Image shown in (A) is the center image, $t=26$ th frame. (C) Mask from composite image combining (A) and (B) and masking with a fuzzy clustering method.

ball method for the brightfield image. The size of the disk used to remove the background can be set with the parameter NEsbdisksize.

The composite image serves as a basis to create a binary mask. Here a fuzzy clustering method is used to segment the image into background and network [76], see Figure 7. The advantage here in contrast to a fixed threshold, i.e. characteristic intensity value, is an adaptive threshold that does not have to be optimized for each data set. The method sorts pixels depending on a defining quantity into similar clusters. Here the defining quantity is the intensity given by the composite image. A number of cluster have to be defined initially (NEfcmclustersnb) and for each pixel a random probability is attributed to be part of a specific cluster. Note that the image can be split into subimages with a width and height given by NErasterSize if one wants to get spatially segmented results which are subsequently concatenated. This may have advantages over a higher cluster number in heterogeneous networks. In either case the cluster centers - averages are computed iteratively and subsequently the probability of a pixel to be part of a certain cluster given its intensity is recalculated. Therefore the cluster centers slowly drift apart grouping pixels of similar intensity. This is done without any spatial correlation. In the experiments shown here a cluster number of 3 always resulted in one cluster of background and 2 clusters of slime mold, see Figure $7(\mathrm{D}-\mathrm{F})$. At the same time increasing the cluster number can putatively yield a higher resolution, especially if more than one quantity is taken into account, but has to be scrutinized for 


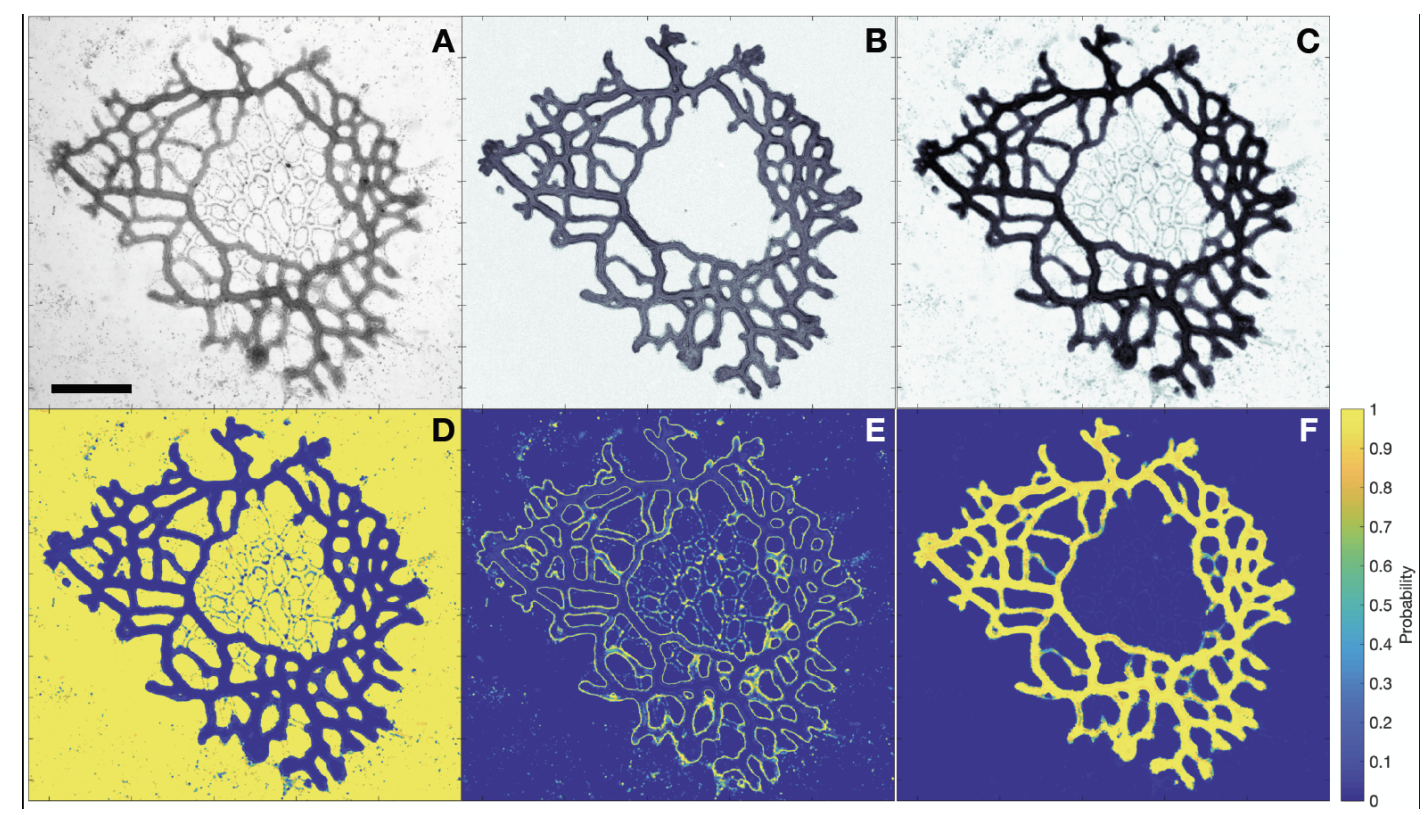

Figure 7: Comparison of the brightfield image, variance and composite image with the results from a fuzzy clustering method using 3 clusters. (A) Exemplary brightfield image of Physarum polycephalumnetwork. Scale bar = $1000 \mu \mathrm{m}$. (B) Logarithm of the variance. (C) Composite image scaled from o to 255. (D) Probability of pixels in (C) belonging to first cluster with the lowest center value of 43. Shows background. (E) Second cluster with center value of 160 . Shows a combination of wall, low intensity network and granules. (F) Third cluster with center value of 246. Shows active network only. Commonly only one of the clusters is removed as background (here D), to retain also low intensity tubes and is subsequently corrected for artifacts (see text).

its computational cost. The program cuts of a number of clusters starting with the highest intensity given by NEfcmclustersmaxnb and regards all remaining pixels as part of the network. The resulting image is a binary image outlining the network called mask.

The mask is enhanced further, i.e. only the biggest structure is considered, small holes are filled and single-pixel edges are smoothed. Subsequently, the resulting mask is used as a template for extracting the network's skeleton with a thinning method [77], see Figure 5. In the skeletonized mask each pixel can be understood as a data point representing local bright field intensity and diameter. From here on intensity means the bright field intensity and not the composite intensity. First, to represent network topology, the network is broken down into vertices and edges where vertices describe pixel positions of branching points and edges rep- 
resent two connected vertices. Each edge then acts as a parent for one specific branch. Iteratively each data point is attributed to a specific network branch. Local diameter is calculated as the largest fitting disk radius around each point within the composite mask. Within this disk the average intensity is computed and saved as the measured intensity at the considered data point. Intensity and diameter anti-correlate due to the optical density of the slime mould and can therefore be used interchangeably considering Beer-Lambert law. In this sense edges are abstracted simple connections and branches represent pixel-based resolution of a tube. The representation gets further broken down with a spline through each branch and a datapoint every $n$ pixels defined by the parameter NEspacing. This guarantees equidistant data points and a finer resolution. In this way each the edges, vertices, diameters, and intensities of each image are extracted which can be done in a computationally parallel fashion.

After the network is extracted in space the quantities in time. To map intensity and diameter over time a reference skeleton is used usually from an early time point. Alternatively a combined image of all skeleton can act as a master mask whose skeleton may be included as the master skeleton (STglobalskelpath, path of sub-pixel resolution file). For every data point the shortest distance to any pixel in the reference image is calculated. This gives a quasi-static $(\mathrm{x}, \mathrm{y}, \mathrm{t}) \rightarrow$ (intensity, diameter) dataset, i.e. branch and vertex positions stay the same but location where intensity and diameter are drawn from can vary. This is justified as long as growth of the organism and vertex movement is minimal. The maximal drawing distance can be set by STmaxdist.

In conclusion the resulting data is the time-evolution of the network diameter or the intensity of brightfield images at a given pixel position. The position data is saved separately while the diameter and intensity is given in a kymograph fashion (3D matrix). This has to taken into consideration when handling the data: neighbouring data points in the kymograph are only adjacent in time not in space.

\subsection{Signal processing on slime mold contractions}

Even though both diameter and intensity are available in the following all results on the contractions are derived from the intensity analysis if not stated otherwise. The diameter has a lower resolution than the intensity and does not show the oscillations when the variance option is used. At 


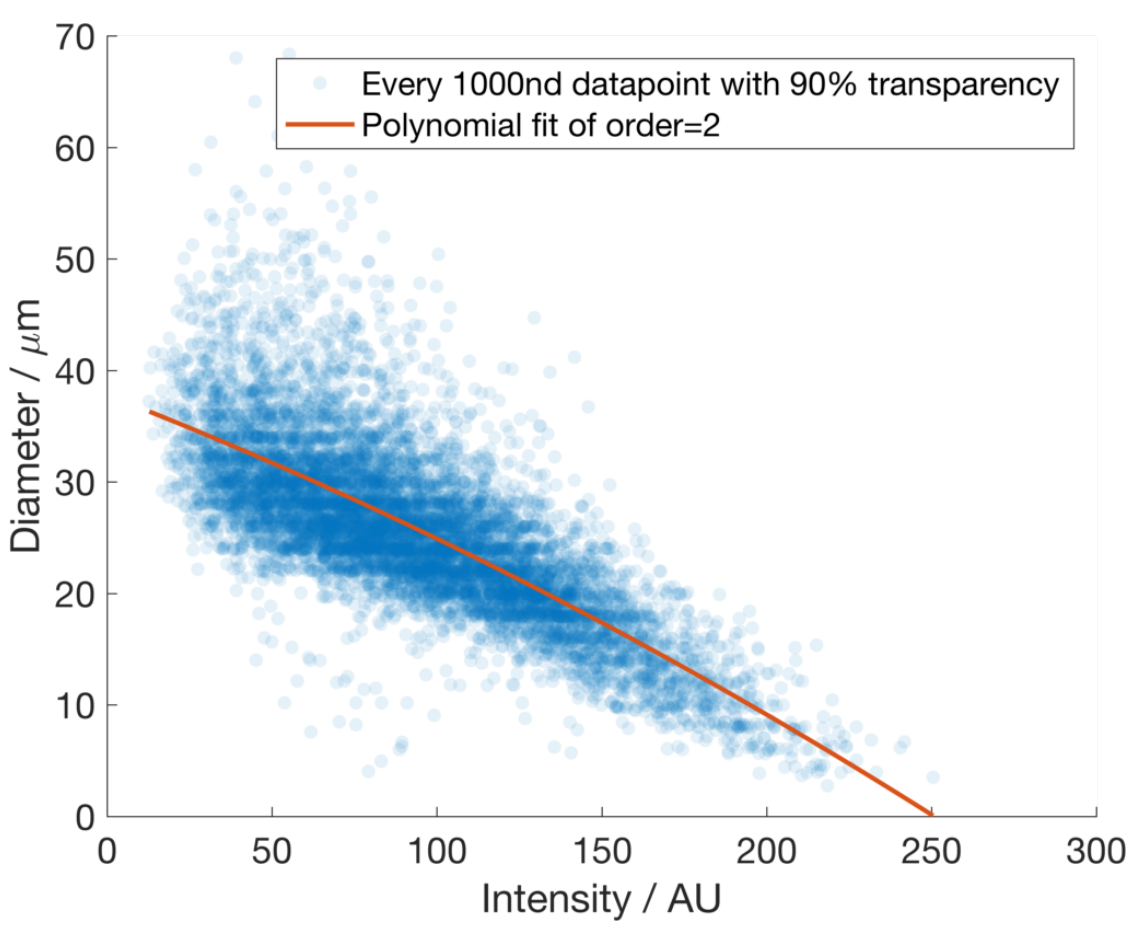

Figure 8: Calibration curve used to calibrate intensity with diameter data. In blue the data from the network shown in Figure 7 is shown with a transparency to highlight abundant regions. In red a polynomial fitting curve of second order used to calibrate the data. Due to the high variability in data a polynomial is used instead of the Beer-Lambert law to better represent the phenomenological basis, i.e. vanishing tubes, intensity cutoffs or non-homogeneous tubes among others.

the same time the diameter data is used to calibrate the intensity data to physical values, see Figure 8.

The oscillatory behaviour of tubes in a certain time window can be described by four time dependent variables, namely amplitude $A$, frequency $f$ (or period $P$ ), phase $\varphi$ and trend (base diameter) $d$. To obtain these the intensity data (from here on called contraction signal) has to be reduced to the oscillatory patterns. The contraction trend $H_{0}(t)$ over time which gives the base radius, see Equation 1, is obtained with a moving-average filter with a kernel width of $200 \mathrm{~s}$ on each time trace. It should be noted here that its assumed that the base radius does not change for the theoretical considerations. This assumptions is justified as long as the time window of adaption (mass transport) is much larger than the oscillation period. The trend is removed from the signal and smoothed with a Gaussian using a kernel width dependent on the oscillation period. The kernel widths are chosen to extract the characteristic contraction pattern which usually have 
a frequency of $\sim 60 \mathrm{~s}$ to $180 \mathrm{~s}$. The values at every data point are stored as a complex valued time array, with the detrended and smoothed intensity representing the real part and the corresponding Hilbert transform representing the complex part, see A.3 for more details. This time array, denoted analytic signal, serves as a basis to get instantaneous phase, frequency and amplitude by computing the angle or absolute value of the complex time series.

Finally, the results can be mapped back onto the network structure for each time point. In this fashion one can follow oscillatory behaviour resolved in time and space. Furthermore, the maps can be clustered in subnetworks and averaged separately to pinpoint local events in time. It should be mentioned that averaging of results for line plots is always done after the data-point based analysis took place.

\subsection{Phase difference determination}

The phase difference between the first and second harmonic of the contraction pattern proves to be a crucial parameter to alter the pumping efficiency of a peristaltic pump. With respect to Equation 2 it corresponds to the difference between the $\varphi_{1}$ and varphi $i_{2}$ giving $\Delta \vartheta=\varphi_{2}-\varphi_{1}$. In the following I want to present how I determine the phase difference from extracted and segmented Physarum polycephalumnetwork data.

The obtained data from the network extraction, see above, is prone to temporal and spatial noise. Reasons for the noise include opaque particles in the tubes, deposited food granules, pixel mismatches from the stitching routine or fluctuations in wall thickness of the tube among others. To reduce the spatial noise the contraction signal is smoothed in a local disk as phase patterns usually occur on larger length scales [28]. Each pixel is recalculated from the values of each pixel in a pixel surrounding defined by dSmoothSize weighted with a Gaussian by the distance to the center. Here values of $30 \mathrm{px}$ were used if not stated otherwise. This recalculation also applies to pixels which were not matched in the stitching procedure (NaN value) if there are more than $25 \%$ of viable pixels in the surrounding. At the same time some experiments show clustering of short connections when the mask shows a high number of holes, i.e. the intensity profile is low in a given region. To remove this artifact one can choose to remove branches with less than $n$ pixels where is $n$ is given by the parameter dNumPtsBranchCutoff. 
Furthermore the data is high-passed in time omitting growth and pruning to isolate the contraction pattern. The dominant $f_{P}$ (prime) and the second harmonic frequency are determined to create band-pass filters for the respective frequency bands in the contractions, see Section A.5.1 for exemplary frequency spectra. With the Hilbert transform we determine the primary frequency $f_{P}$ of the contraction pattern for the whole dataset. The frequency does change over the course of an $3 \mathrm{~h}$ experiment - and crucially has to do so for phase adaptation to happen - yet the changes are small and happen within the chosen spectral band. The primary frequency $f_{P}$ is used as the baseline to band-pass filter the first $f_{1}=f_{P}$ and second harmonic $f_{2}=2 f_{P}$. In this way, for every point in space, the time series is split into the respective first and second harmonic. The filter kernels are created with the Matlab function filterDesigner for the high-pass and the dominant and for the second harmonic band-pass respectively. For the filters I use Kaiser windows as the tapering function and orders between $n=50$ to 150 to balance resolution with accuracy. A higher order results in a steeper tapering but results in a loss of time points. When using the code on a cluster computing machine the filters have to be created beforehand on a local machine and made available to the cluster in a .mat file at a location given with the parameter dFilterLoc.

The filtered data is then analyzed iteratively for each data point in space individually. The dominant wave and the second harmonic wave contents are fitted in a step-wise manner to a fitting function $F_{1 / 2}(t, \varphi)=\sin \left(2 \pi f_{1 / 2} t+\right.$ $\varphi$ ), with $f_{1 / 2}$ the frequency of the first or second harmonic, $t$ the time and $\varphi$ the phase. The step width is chosen as two periods of the main frequency but can alternatively set manually by the parameter dApproxWindow. Furthermore the data is divided by its amplitude and then fitted to the fit function determining the phase $\varphi$ with the Matlab function Isqnonlin. The code offers a debugging option here where insufficient filters can be detected. Furthermore the fit residuals and the local amplitudes are saved which are good indicators for the goodness of the estimation. Those values can also be utilized to identify local contraction patterns, e.g. a high residual can indicate a local phase jump or a low amplitude ratio between the second harmonic and the first harmonic indicates a recently restarted contraction rhythm [32]. The resulting phases now give the phase difference $\Delta \vartheta=\varphi_{2}-2 \varphi_{1}$ in the reference system of the second harmonic and serves as an experimental basis for chapter 6 . 



\section{Part III}

\section{RESULTS}

In the following two result sections are presented. Both are paper manuscript and are left as is, hence include introduction and discussion albeit shorter.

The first presented paper is published in Journal of Physics D: Applied Physics ${ }^{75}$ and the pre-print is included here. It is a co-first authored paper with Mirna Kramar (MK). I designed all figures and did the data analysis for the publication. MK and me equally contributed to the writing with revisions by Karen Alim (KA). MK, KA and me collectively designed the study. MK performed the experiments.

The second paper is in review. I performed the experiments, did the data analysis, reviewed the analytical work, co-designed the study and wrote the paper. Stefan Karpitschka (SK) added the elastic deformation energy and perturbative approach to a fluid filled visco-elastic tube. KA revised the manuscript and co-designed the study. We equally contributed to the design of the study through fruitful discussions. 



\subsection{Abstract}

Wounding is a severe impairment of function, especially for an exposed organism like the network-forming true slime mould Physarum polycephalum. The tubular network making up the organism's body plan is entirely interconnected and shares a common cytoplasm. Oscillatory contractions of the enclosing tube walls drive the shuttle streaming of the cytoplasm. Cytoplasmic flows underlie the reorganization of the network for example by movement toward attractive stimuli or away from repellants. Here, we follow the reorganization of $\mathrm{P}$. polycephalum networks after severe wounding. Spatial mapping of the contraction changes in response to wounding reveal a multi-step pattern. Phases of increased activity alternate with cessation of contractions and stalling of flows, giving rise to coordinated transport and growth at the severing site. Overall, severing surprisingly acts like an attractive stimulus enabling healing of severed tubes. The reproducible cessation of contractions arising during this wound-healing response may open up new venues to investigate the biochemical wiring underlying Physarum polycephalum's complex behaviours.

\subsection{Introduction}

Simple organisms like fungi and slime moulds are able to display complex behaviours. This is surprising given that their network-like body plan lacks any central organizing centre. The slime mould Physarum polycephalum has emerged as a model system to study the complex dynamics these organisms use to adapt to their environment. The organism has been shown to find the shortest path through a maze ${ }^{78}$ and connect food sources in an efficient and at the same time robust network comparable to man-made transport networks ${ }^{3}$. Furthermore, the slime mould distributes its body mass among several resources to obtain an optimal diet ${ }^{4}$ and is able to anticipate recurring stimuli 5 . 


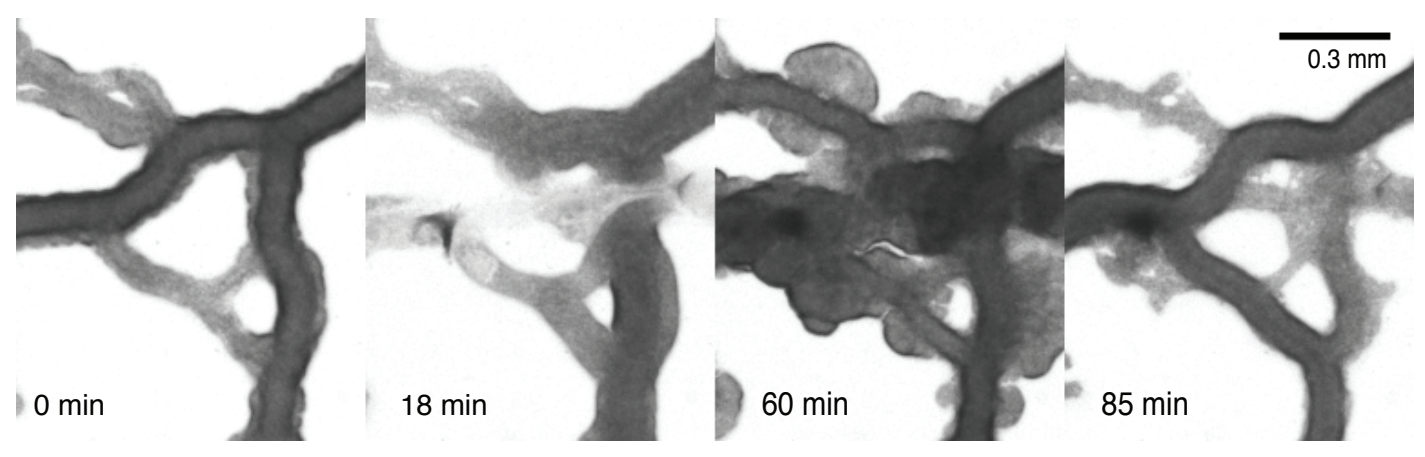

Figure 9: Wound healing process in P. polycephalum illustrated at four time points using bright field images. The cut occurred at $18 \mathrm{~min}$ and the fan grown at cut site reached its maximal size at $60 \mathrm{~min}$. The network morphology was restored after $85 \mathrm{~min}$.

P. polycephalum is a true slime mould that forms a plasmodial network. Nuclei keep on dividing without forming cell walls, which results in a syncytial web-like network. The cytoplasm within this tubular network flows back and forth in a shuttle flow ${ }^{35}$. These cytoplasmic flows are driven by cross-sectional contractions of the actin-myosin meshwork lining the gellike tube walls ${ }^{27}$. Flows are organized across the entire network in a peristaltic wave of contractions that matches organism size ${ }^{28}$. Flows generated in the organism are optimized for transport as contractions increase the effective dispersion of particles way beyond molecular diffusivity by a mechanism called Taylor dispersion ${ }^{33}$.

P. polycephalum adapts its network-like morphology to its environment by chemotaxis ${ }^{79-81}$. Here, stimulants are classified by being an attractant or a repellant depending on the organism's response to migrate toward or away from the stimulant. Stimulants have also been shown to affect cross-sectional contractions organism-wide by an increase in their frequency and amplitude for an attractant or a decrease for a repellant ${ }^{82,83}$. A variety of chemical stimuli have been discussed for P. polycephalum, with glucose being a prominent attractant and salts like $\mathrm{NaCl}$ being effective repellants ${ }^{16,84,85}$. Temperature ${ }^{86,87}$ and light ${ }^{88,89}$ have also been found to act as stimulants that trigger organism-wide restructuring of the transport networks' morphology. In fact, the cytoplasmic flows themselves serve as the medium by which stimuli pervade the organism ${ }^{31}$.

A lot less is known about the impact of mechanical perturbations on the organism. In its natural habitat the slime mould suffers predation from grazing invertebrates causing severing that disrupts the transport network 
and its cytoplasmic flows. In experiments it has been found that quickly stretching a strand to $10-20 \%$ of its length while keeping it intact increases the amplitude of oscillations ${ }^{90}$. Excising a single strand from a plasmodial network has been observed to lead to a roughly 20 minute cessation of contractions in the strand until recovery ${ }^{91}$. This phenomenon was not observed for strands excised from the growing fan region of the slime mould resulting in speculations about the motive force being limited to the fan only. Yet, the cessation of contractions turned out to be hard to reproduce, see $^{92}$ and references therein. Among these discordant observations what remains established is local gelation of cytoplasmic flows upon touch without severing the organism ${ }^{93}$. Despite the limited knowledge, wounding the organism by severing the network is part of daily laboratory routines and an eminent perturbation in natural habitat.

Here we investigate P. polycephalum's dynamics during wound healing following the quick and complete severing of a tube within the organism's network. We follow the process of wound healing across the individual's entire body, over the course of one hour after severing. The exemplary quantitative analysis of organism-wide contractions reveals a stepwise response spanning four different states. Briefly after severing, the contractions are often marked by an increase in amplitude and frequency, followed by a several minutes long cessation of contractions and stalling of cytoplasmic flows. This resting state is terminated by a sudden restart of vigorous contractions as the severed tube re-fuses. The vigorous state then transitions into a state of network-spanning contractions and continuous fan growth at the wounding site until the organism reverts back to pre-stimulus dynamics. Timing and significance of individual steps varies with the severity of cutting and cutting site location within the network. For example, stalling is found to be less pronounced when the network is cut in fan-like region. Overall, quick and complete severing triggers a response pattern with characteristics of the response to an attractive stimulus, including an increase in amplitude and frequency and net movement to stimulus site, see Fig. 9. The reproducibility of stalling clarifies earlier contradictions and at the same time opens new avenues to investigate the biochemical dynamics behind the highly coordinated acto-myosin contractions underlying P. polycephalum's arguably fascinating dynamics. S 


\subsection{Methods}

\subsubsection{Culturing and data acquisition}

The plasmodium is prepared from microplasmodia grown in liquid medium. The recipe for the medium is inspired by ${ }^{74}$, see Sec. 3. The advantage of this method over growing the plasmodium on oat flakes or bacteria is the ability to precisely control the nutritional state and amount of the organism. Also, plasmodia grown this way are free from oat flake residues or vacuoles containing food, which provides a cleaner sample for imaging. To prepare the plate for imaging, $0.2-0.5 \mathrm{~mL}$ of the microplasmodia grown in a shaking culture at $30^{\circ} \mathrm{C}$ are transferred to an $1.5 \%$ agar plate and stored in a closed, but not sealed dish in the dark. After 12-24 hours, the microplasmodia fuse into a single plasmodium. The plasmodium is ready for imaging when there are no visible traces of liquid medium and the organism assumed its characteristic network shape, which usually occurs up to 36 hours after plating.

Imaging is done with a Zeiss Axio Zoom V.16 microscope, equipped with a Zeiss PlanNeoFluar 1x/0.25 objective and a Hamamatsu ORCA-Flash 4.0 digital camera. A green filter (550/50nm) is placed over the transmission light source of the microscope to diminish $P$. polycephalum's response to the light, and a humidity chamber prevents the sample from drying out. The acquisition of the images is done in Zeiss ZEN 2 (Blue Edition) software with bright-field setting. During the acquisition, the illumination of the sample is kept constant, and an image is taken every 3 seconds. The plasmodium is imaged for $\sim 1$ hour before the application of the mechanical stimulus to allow for the accommodation to the light ${ }^{80}$. The stimulus is applied manually, using a microinjection needle with a blunt tip. The needle tip is held above the surface of the agar at a small angle and quickly dragged across the chosen plasmodial tube. The cut is severe and complete if the two parts of the tube separate completely. The plasmodium is then further imaged for more than 1 hour.

Using microplasmodia is so far the optimal way of obtaining non-severed networks, where the size and nutritional state are reproducible. However, there are challenges during the imaging that decrease the reproducibility of the experiment. In particular, plasmodia are highly motile and change their morphology accordingly. Furthermore, the organism tends to develop very large foraging fronts, which are not a suitable input for the presented 
comprehensive data analysis as they lack network characteristics. Lastly, the microscope light can act as stimulus ${ }^{3,88,89}$, and even the green-filtered low-intensity illumination may cause the network to respond and change its behaviour to escape the imaging region. These challenges combined make the reproducibility and required stability of the network morphology over time challenging.

\subsubsection{Comprehensive network-based contraction analysis}

To quantify contraction dynamics we analyse bright field recordings in two different ways: for two morphologically static networks (see E2 and E3 in the experiment list) we perform an exhaustive network-based analysis as outlined in the following (see Fig. 13 and Fig. A29). For the additional 19 specimen which alter their network morphology dramatically over the course of the experiment, we analyse kymographs along static parts of the network as described in detail in Sec. A.4 (see exemplary E1 and Mov. M5).

Images recorded as a time series are processed as 8-bit uncompressed TIFs. At first every image is processed separately, then the results are stitched together, largely following Ref. ${ }^{28}$, and lastly the collective is analysed. On every single image, background is removed with the rolling-ball method. Then the image is used to create a mask, a binary image, with an intensity threshold that separates the network from the background. The mask is enhanced further, i.e. only the biggest structure is considered, small holes are filled and single-pixel edges are smoothed. Subsequently, the resulting mask is used as a template for extracting the network's skeleton with a thinning method. In the skeletonized mask each pixel can be understood as a data point representing local intensity and diameter (see Fig. 10). Local diameter is calculated as the largest fitting disk radius around the point within the mask. Within this disk the average intensity is computed and saved as intensity at the considered data point. Intensity and diameter anti-correlate due to the optical density of the slime mould and can therefore be used interchangeably considering BeerLambert law. Individual data points are attributed to a specific network branch of the network skeleton. To represent network topology, the network is broken down into vertices and edges where vertices describe pixel positions of branching points and edges represent two connected vertices. Each edge then acts as a parent for one specific branch. In this sense edges 


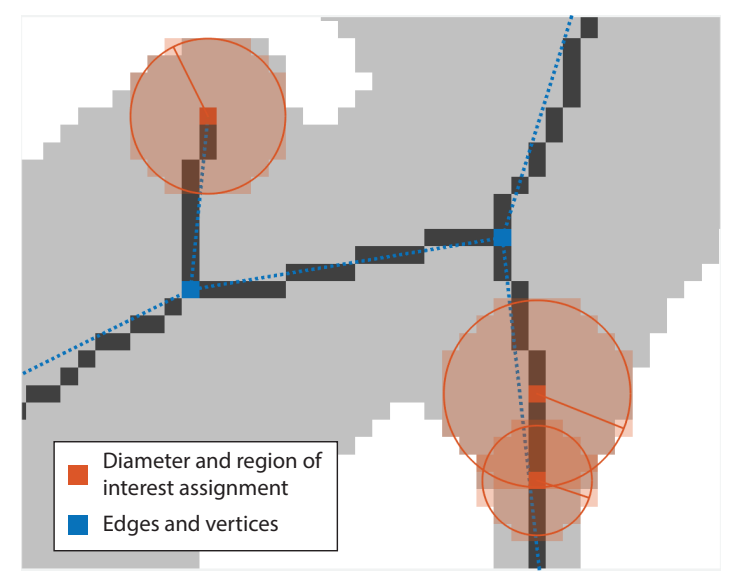

Figure 10: Scheme of intensity and diameter data extraction based on P. polycephalum bright field images. The light grey area depicts the network mask based on the bright field images. Dark grey lines represent the network skeleton and the corresponding topology is shown in blue. Each pixel of the skeleton acts as a reference point for data derived during the analysis. The diameter is set as the distance from the reference point to the next non-mask pixel. The intensity is calculated by averaging individual pixel intensities over a corresponding disk (red).

are abstracted simple connections and branches represent pixel-based resolution of a tube.

After the network is extracted in space, the edges, vertices, diameters, and intensities are concatenated in time. To map intensity and diameter over time, a reference image is used, usually from an early time point. For every data point the shortest distance to any pixel in the reference image is calculated. This gives a quasi-static $(\mathrm{x}, \mathrm{y}, \mathrm{t}) \rightarrow$ (intensity, diameter) dataset, i.e. the topology and vertex positions stay the same, but intensity and diameter can vary. This is justified as long as growth of the organism and vertex movement is minimal. The oscillatory behaviour of tubes in a certain time window can be described by four time dependent variables, namely amplitude $A$, frequency $f$ (or period $P$ ), phase $\varphi$ and trend (base diameter) $d$. Each can be calculated from the time-evolution of the diameter or the intensity data, but if not stated otherwise the following results are only derived from intensity analysis.

The trend $d(t)$ is obtained with a moving-average filter with a kernel width of $200 \mathrm{~s}$ on each time trace (see Fig. 11). The dataset is detrended with the calculated trend and smoothed with a Gaussian using a kernel width of $39 \mathrm{~s}$. The kernel widths were chosen to extract the characteristic contraction pattern which usually has a frequency of $\sim 90 \mathrm{~s}$. The values at every 
data point are stored as a complex valued time array, with the detrended and smoothed intensity representing the real part and the corresponding Hilbert transform representing the complex part, see A.3 for more details. This time array, denoted analytic signal, serves as a basis to get instantaneous phase, frequency and amplitude by computing the angle or absolute value of the complex time series. Finally, the results are mapped back onto the network structure for each time point. In this fashion one can follow oscillatory behaviour resolved in time and space. Furthermore, the maps can be clustered in sub-networks and averaged separately to pinpoint local events in time. It should be mentioned that averaging of results for line plots, i.e. Fig. 13, is always done after the data-point based analysis took place. In this way for example, the apparent amplitude of the averaged intensity (Fig. 13D) can be lower than the amplitude of each data point averaged (Fig. 13B).

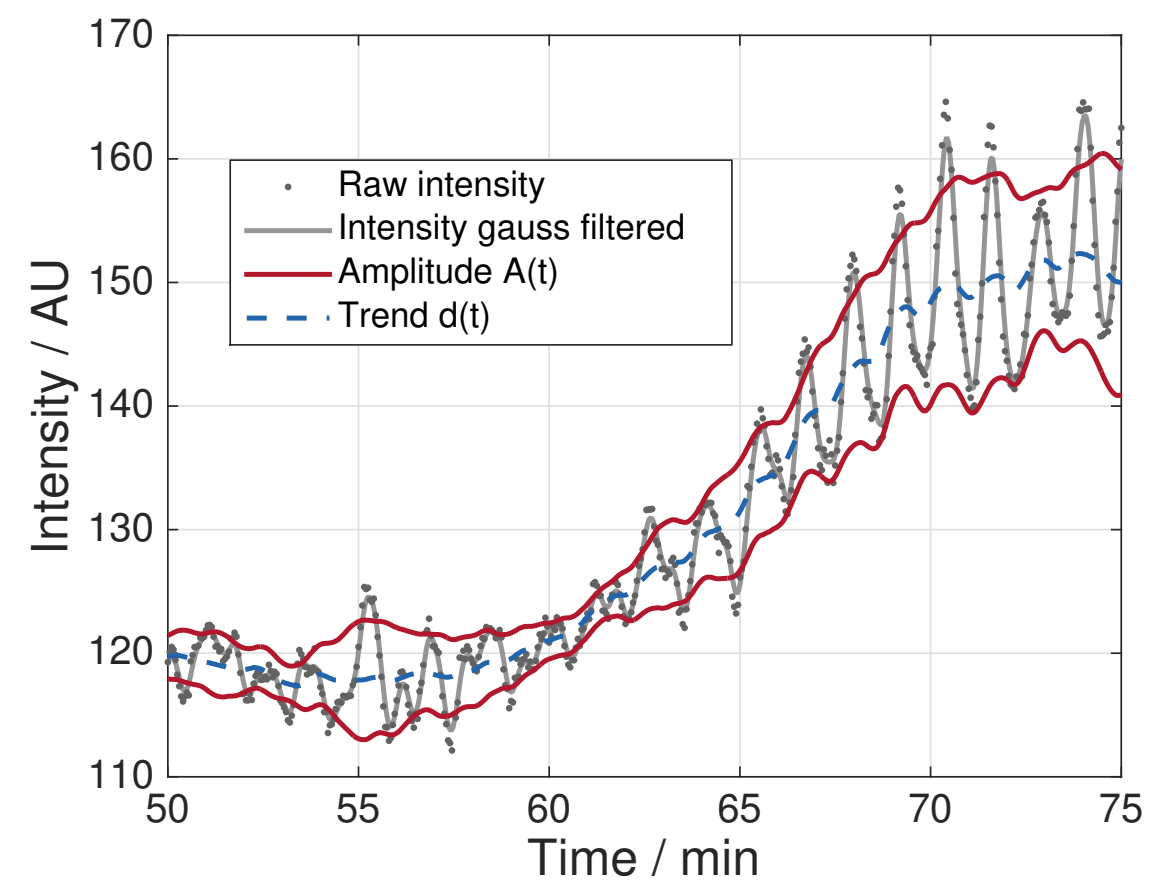

Figure 11: Derivation of oscillation specific parameters, i.e. amplitude $A(\mathrm{t})$, frequency $f(\mathrm{t})$ and trend $d(\mathrm{t})$, from single pixel time series. The trend is calculated using a moving average with a kernel width of $200 \mathrm{~s}$. Intensity is filtered with a Gaussian of width $39 \mathrm{~s}$. Amplitude and frequency are calculated from the absolute value and angle of the complex-valued analytic signal, respectively. 


\subsection{Results}

\subsubsection{Wounding induces fan growth at cut site}

We observe specimens before and after a quick and complete severing of a tube to follow the response of P. polycephalum to wounding (see Fig. 12A, Mov. M1 and Mov. M5). Bright field movies reveal that cutting of main tubes distal to fans triggers cessation of contractions followed by stalling of cytoplasmic flow ( $\mathrm{n}=15$ out of 21). After contractions resume the severed tube fuses back together ( $\mathrm{n}=21$ out of 21), i.e. flow is re-established, and a fan starts to grow at the cut site. Furthermore, we observe accumulation of body mass close to the cut site which is most prominent in peripheral cuts (Fig. A28). However, the growth is transient and after a given time the initial morphology is restored and the organism returns to typical behaviour comparable to before wounding.

In consideration of previously mentioned technical limits, we selected one representative dataset with prominent discernible features for networkbased analysis. The following findings are derived from this dataset and later compared with other experiments. The specific timing of events in the representative data set is as follows (see Fig. 12). Two tubes are severed at 17.3 min effectively dividing the network into two parts. In both subnetworks, the size-wise bigger and smaller part, flows stall transiently around $30 \mathrm{~min}$. At $38 \mathrm{~min}$ a connecting tube is reinstated and starts to reestablish cytoplasmic flows across the cut site. Until about $63 \mathrm{~min}$ a transient fan is created at the cut site. At 90 min the initial morphology is restored and fans are grown elsewhere.

\subsubsection{Spatial mapping reveals localized stalling}

We perform network-based analysis on the wounded specimen to extract the interplay of contractions during the healing response. In particular, we map out the amplitude and frequency of contractions spatially (see Fig. 12, Mov. M2 and Mov. M3). This allows us to exactly localize the onset of stalling as it goes hand in hand with low values of amplitude and frequency. Likewise, patterns in contraction dynamics in a region of interest are iden- 


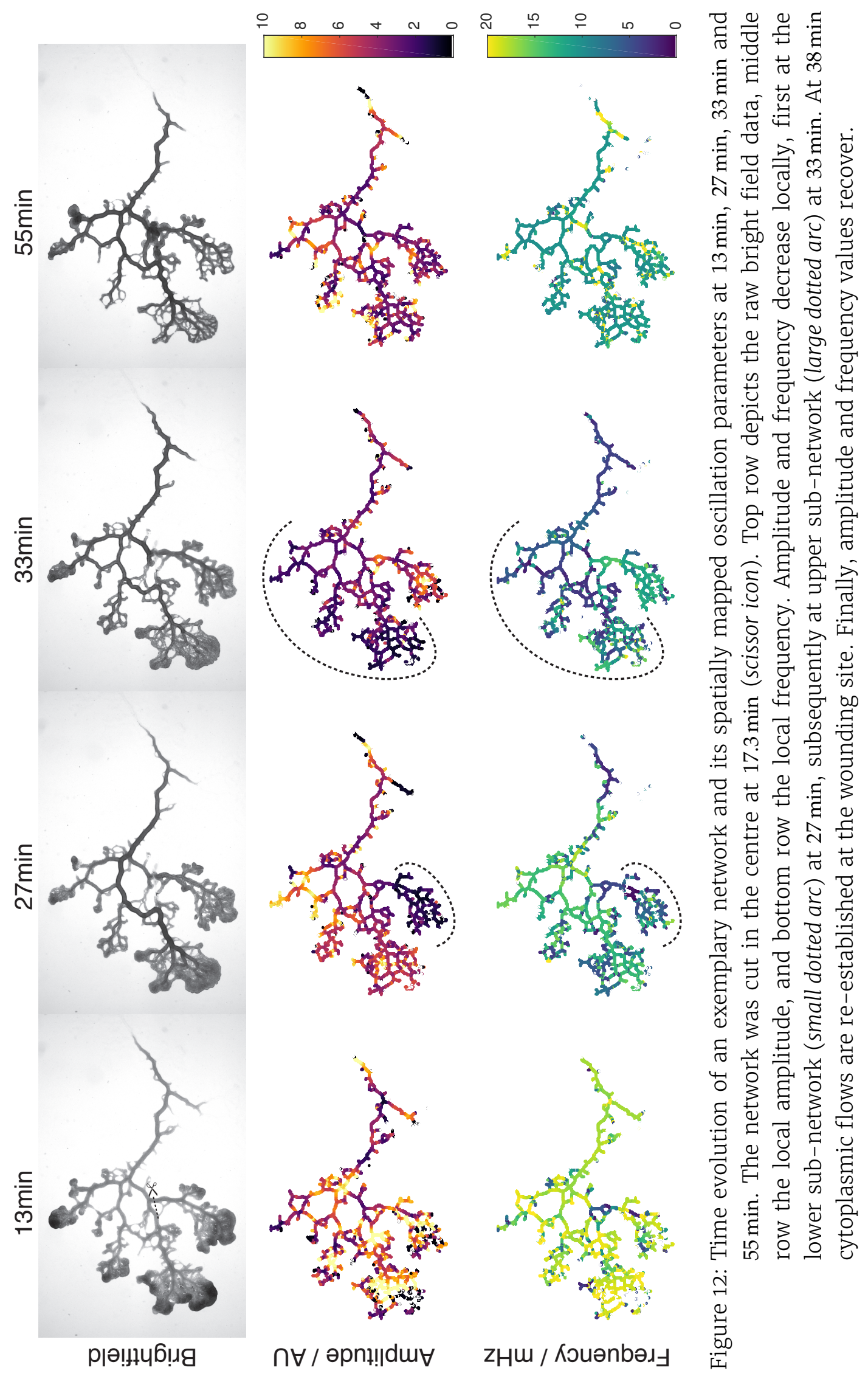


tified by spatially averaging amplitude and frequency in this region (see Fig. 13).

In the representative dataset, wounding separates the network into two sub-networks. Spatial mapping reveals that oscillations cease on different time-scales in the two sub-networks. By identifying the two sub-networks as separate regions of interest, we quantify the patterns in contraction taking the spatial average of the respective contraction variables in each region. The small sub-network shows a drop in amplitude at $21.5 \mathrm{~min}$ by $63 \%$ and only recovers eight and a half minutes later to comparable values. Here, the percentage is given as ratio of time averages before, during and after stalling. In detail, the averages of the first 215 minutes, the 95 minutes during stalling and 15 minutes after stalling were considered. The bigger sub-network drops significantly later at $28 \mathrm{~min}$ by $51 \%$ and recovers to $29 \%$ below the initial value nine minutes later. In the same time frames the frequency drops by $32 \%$ and $45 \%$ for the small and big sub-network, respectively. Yet, neither sub-network recovers its frequency fully right after the stalling phase. Only the small sub-network recovers 35 minutes later to initial frequencies whereas the bigger region levels off $35 \%$ below the initial value.

Furthermore, the phase patterns over time (see Mov. M4) reveal changes in the travelling waves upon cutting. Initially (o to $17.3 \mathrm{~min}$ ) one can observe peristaltic waves from the tail (right-hand side) to the front (left-hand side) which finally merge into concentric patterns in the fan regions. Then, at 18 to $30 \mathrm{~min}$, the small sub-network slows down noticeably (see change in frequency) and the big sub-network contracts with less apparent spatial correlation, i.e. the peristaltic wave pattern is temporarily lost.

\subsubsection{Fan growth phase coincides with stable network-spanning contractions}

After re-fusing of the two sub-networks, another distinct phase characterized by stable network-spanning contraction dynamics can be observed. In Fig. 13D contractions appear uniform from $44 \mathrm{~min}$ until $63 \mathrm{~min}$. During this phase, amplitude and frequency level off to a stable value with little fluctuations. The small sub-network shows a slight increase in frequency over this period and has more fluctuations in the average intensity data than the big sub-network. Note, that the time frame of these contractions coincide with fan formation at the cut site. Furthermore, the end of this phase also coincides with the largest fan in respect to area. 
Network-spanning contractions are further supported by the phase time series. When considering the phase development one can already observe a peristaltic wave travelling towards the cut site in the small sub-network as early as $30 \mathrm{~min}$. A spanning pattern in the large sub-network is reinstated around the 35 min mark and a global pattern (small and large subnetwork) appears roughly three minutes after re-fusing ( $40 \mathrm{~min}$ ). Then a standing wave pattern appears between the central region including the cut site and the periphery. It is stable and network-spanning until $63 \mathrm{~min}$. Subsequently the phase pattern breaks into a peristaltic wave similar to pre-cut and propagates from the tail and the small sub-network into fan regions in the large sub-network.

\subsubsection{Stalling and fan growth periods are bridged by distinct transition periods}

Closer analysis of contraction dynamics over time reveals that the time point of the cut, the stalling phase and the fan growth phase are transitioned by phases of high fluctuations. Particularly in the presented case, before stalling occurs, amplitude and frequency peak shortly in both subnetworks (see arrows in Fig. 13). In the small sub-network this peak coincides with the cut, whereas another ten minutes pass for the big subnetwork before the amplitude reaches its maximum. Surprisingly, here the frequency decline occurs three minutes before the amplitude drops. After stalling the amplitude increases sharply in both sub-networks, yet stays below previous values in the big sub-network. The small network undergoes a phase of roughly $13 \mathrm{~min}$ where the amplitude oscillates vigorously. This also coincides with a second frequency drop even though there is no apparent drop in amplitude at this time point. After the fan growth phase, amplitude and frequency show slight gradients once more. Here behaviour becomes comparable to the pre-cut state as the slime mould develops a preferred growth direction in the periphery and continues foraging.

\subsubsection{Fan creation and stalling is reproducible for complete severing}

For comparison we analysed a second dataset with the same networkbased method (see Fig. A29). The key features, i.e. cut repair, stalling, a transition phase, stable network-spanning contractions and return to pre-cut behaviour are found likewise, but the succession and timing of the 


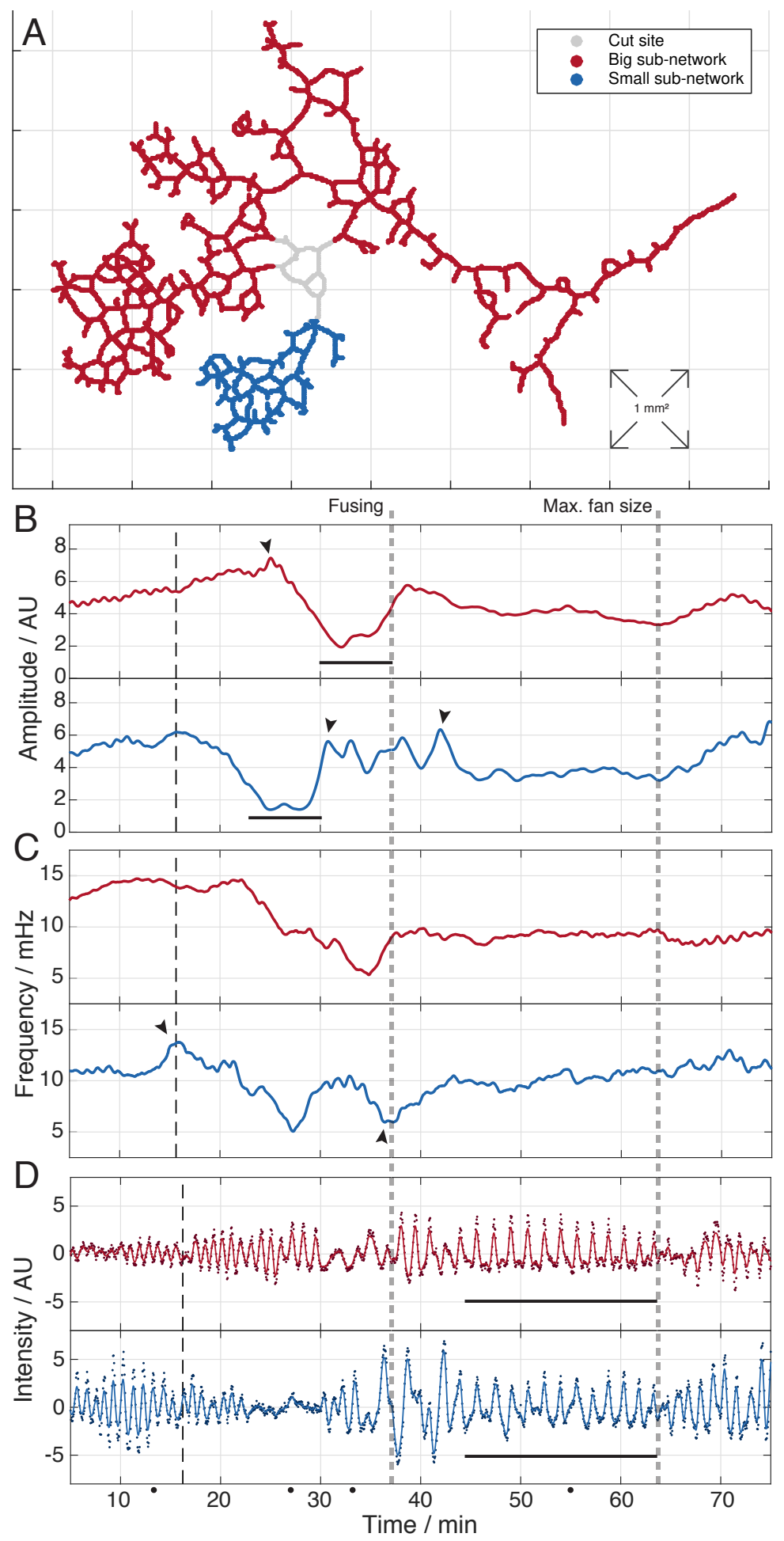

Figure 13: Comparison of oscillation parameters in the big- and small-sub-network depicted in (A) which result from the cut. Grey area (cut site) is not considered in the analysis. Time series of amplitude (B), frequency $(\mathrm{C})$ and intensity (D) are averaged in the respective domains and compared; top : big subnetwork, bottom: small sub-network. In each of these plots the black dashed line indicates the moment of the cut. The first grey dashed line marks the time point of fusion and the second the moment of maximal fan size. Black bars underline periods of stalling in (B) and fan growth in (D). In (D) the solid line represents the Gaussian filtered intensity (kernel width $=39 \mathrm{~s}$ ) and markers show raw averaged data. Black arrows indicate respective extremal peaks in the transition periods. Four black dots on the time line correspond to the four time points chosen in Fig.12. 
specific events vary. This dataset has a weaker fan growth at the cut site and the time point of maximal fan size follows immediately after fusion. Given the short period of fan growth global network-spanning contractions are not observed. However, standing phase wave patterns are visible in the larger sub-network before fusion. Lastly, the transition phase shows peaking amplitude and fluctuating frequencies and reverberates for more than 30 minutes. At $70 \mathrm{~min}$ the network reinstates a peristaltic wave toward peripheral fan regions resuming pre-cut dynamics.

In further experiments analysed with a kymograph based approach, we confirmed stalling to be a common response after a cut (see Fig. A.6, n=15 out of 21). However, the degree and duration of stalling is varying between experiments and is most reproducible for a severe cut close to the centre of the network.

In detail, we observe that both the degree and duration of stalling, depend on the network size and morphology, cut location, possibilities of rerouting the flow through neighbouring tubes and presence of large fans. Also, a network undergoing quick changes in morphology due to a presumed light shock is less likely to show stalling. Varying cut location shows that complete severing of a tube, with a diameter comparably large in size and few neighbouring tubes, results in strong stalling, see experiments $E[2,3,5,6,8,9,12,13$, and 18]. The effect is even more pronounced in smaller networks and on tubes close to the centre of the network $(E[2,3,5$, 8 , and 18]). Stalling is less pronounced, as measured by relative change in amplitude and frequency as well as visual inspection of bright field data, if severing was applied to fan-like regions or peripheral tubes $(E[10,11,14,15$, $16,17,19,20$, and 21]). If a severed tube had alternative routes with a comparable flow direction, neighbouring tubes inflated shortly after the cut, indicating a re-routing of flow. Yet, in this case stalling severity ranged from non-existent (E19) to full-stop (E1). In all data sets fan growth is observed around the cut site, yet duration and fan sized varied greatly (see E2 and E9 as maximal and minimal examples).

In all 15 experiments that show stalling, the period lasted for a minimum of three minutes. The exact time point of stalling onset and its duration varied. Duration of transition periods also varied from complete omission up to 22 minutes between cut and stalling. In 7 out of 15 experiments, a vigorous phase of increase in frequency or amplitude fluctuations could be observed in the transition phases. 


\subsection{Discussion}

We investigate $P$. polycephalum's response to wounding in the form of a quick and complete severing of tubes using bright field microscopy and quantitative analysis of contraction patterns. Mapping out the contractions amplitude and frequency in space and time allows us to uncover a multistep pattern of wound healing in P. polycephalum.

The key of our network-based analysis is mapping contraction variables onto a few pixels serving as the skeletonized backbone of the complete network. This representation allows us to capture contraction dynamics across the entire network over the course of several hours with handleable amount of data. Furthermore, spatial mapping visualizes abstract variables in an approachable way which outlines region of interests or patterns in space. For example, in the representative data set the time-shift in the response pattern between the two sub-domains of the network would have been lost when averaging contraction dynamics across the entire network (see Fig. A27).

Among the multiple steps in the response to wounding the cessation of oscillations and stalling of the cytoplasmic streaming is most striking. The phenomenon of stalling of cytoplasmic flows has been observed previously ${ }^{23,90}$, but its reproducibility was deemed questionable ${ }^{92}$. Our work shows that cut location and severity are crucial parameters for inducing reproducible stalling. The stalling period is omitted when a tube is not completely severed, or cut in a way that allows the cut ends to rejoin quickly. In addition, the specific body plan affects the impact of a cutting stimulus. For example, severed fan-like regions show less pronounced stalling. However, we find reproducible strong stalling in networks where the affected tubes are crucial connections that cannot be re-routed easily - thereby clarifying previously discordant observations.

Stimuli are commonly classified into attractants or repellants. The response of $P$. polycephalum to an attractive stimulus includes fan growth and mass transport towards the stimulus site, often accompanied with an increase of oscillation frequency and amplitude. When we apply a wounding stimulus resulting in complete cutting of a tube, we observe a multistep response pattern where only two out of four steps show a noticeable increase in amplitude and frequency. Yet, wounding implies that the network architecture is perturbed. Taken into account that contraction fre- 
quency decreases as organism size decreases ${ }^{94}$ the impairment of network architecture itself might counteract any increase in frequency. Despite the weak indication from contraction frequency and amplitude, we always observe fan growth and movement of mass toward the cut site regardless of the tube hierarchy, plasmodium size or the severity of the cut. Fan growth is a lot bigger than initial spillage of cytoplasm due to cutting. Furthermore, we often identify a specific fan growth phase of network-spanning contractions well separated in time from the cutting event by the stalling phase. We therefore identify wounding as an attractive stimulus. The observation of network-spanning oscillations during fan outgrowth adds to our confidence about cutting being an attractive stimulus since the observed phase patterns resemble contraction patterns found in earlier work with attractive stimuli using glucose as a stimulant ${ }^{31}$.

Employing spatial data analysis we uncovered that wounding triggers a choreography of multiple successive steps to heal the severed tube. The mere duration of the healing response now defines a suggested minimal wait time after trimming for P. polycephalum experiments. The complexity of the response hints at an intricate signalling pattern underlying the coordination of contractions. It is likely that also the response to classical attractants and repellants, when scrutinized, reveal multiple steps. Unravelling the workings behind P. polycephalum's ability to adapt, is arguably a fascinating albeit challenging question. Here, the reproducible cessation of contractions arising during this wound-healing response may open up new avenues to investigate the biochemical wiring underlying $P$. polycephalum's complex behaviours. Furthermore, it is fascinating that the impact of wounding can be weakened by network architecture. This suggests that P. polycephalum's body plan itself could be part of the organisms strategy to not only adapt to its environment, but also specifically prevent severe consequences of wounding.

\section{Acknowledgements}

We thank Christian Westendorf for instructions on growing microplasmodia, as well as for invaluable discussions and advice. M.K. and F.B. acknowledge support by IMPRS for Physics of Biological and Complex Systems. 

LIVING SYSTEM ADAPTS HARMONICS OF PERISTALTIC WAVE FOR COST-EFFICIENT OPTIMIZATION OF PUMPING PERFORMANCE

\subsection{Abstract}

Wavelike patterns driving transport are ubiquitous in life. Peristaltic pumps are a paradigm of efficient mass transport by contraction driven flows - often limited by energetic constraints. We show that a cost-efficient increase in pumping performance can be achieved by modulating the phase difference between harmonics to increase occlusion. In experiments we find a phase difference shift in the living peristalsis model P. polycephalum as dynamic response to forced mass transport. Our findings provide a novel metric for wavelike patterns and demonstrate the crucial role of nonlinearities in life.

\subsection{Introduction}

Wavelike patterns are ubiquitous in life. They range from everyday phenomena such as gut peristalsis [64], undulatory locomotion of worm-like organisms [95] or the cardiac cycle [96], to microscopic waves, such as ciliary and flagellar beating [97], gene oscillation [98] or reaction-diffusion patterns [99]. The patterns' functions vary, but it is noteworthy that they often relate directly to transport or locomotion. Each system oscillates in distinct characteristics, for example body shape [100] or molecule concentration [101], yet all systems are governed by the limited set of a wave's parameters - wavelength, amplitude, and frequency. Moreover parameter choice is limited by physical or biological constraints. Which strategies does a living system use to fulfill the wave's function given its constraints?

Environmental changes challenge living systems to adapt, forcing alteration of wave dynamics given the limited set of wave parameters while additionally staying within its constraints. As an example, the nematode C.elegans changes its locomotion depending on the viscoelasticity of its environment by modulating undulation wavelength, amplitude, and fre- 
quency of its wave-shaped body [102]. Yet, this adaptation goes hand in hand with changes in the energy cost of the wave, often the most limiting constraint of life [103, 104].

While a sinusoidal wave shape offers few parameters to adjust, some living systems instead use a superposition of waves. Examples are the peristaltic contractions of the human gut [105] or the flagellar beating of human spermatozoa [106]. The superposition of multiple waves allows a modulation of the total wave shape, thereby increasing the parameter space available when responding to environmental changes. In particular, for a set of harmonic waves, we here introduce the phase difference between them as a new parameter. How much control over a waves' total performance resides in the phase difference between superposed harmonic waves given limited wave energy cost?

The paradigm for wave driven mass transport and locomotion is peristaltic pumping $[68,69]$. Here, mass transport is driven by radial contractions traveling along a tube which cause net transport of the enclosed fluid, see Fig. 14. The contractions can be considered as periodic train waves with the characteristic wave parameters defining the flow rate. To this point it has been unclear how peristaltic pumping performance can increase in a cost efficient manner, since changing the wave's symmetry has been shown to have a negligible effect on the pumping performance [107]. To resolve this puzzle we turn to living systems forced to transport mass in response to environmental cues.

The network-forming slime mold Physarum polycephalum has gained broad attention as a living system using peristalsis $[11,28,66]$. Its interconnected tubes contract rhythmically, driving fluid flow throughout the network [28]. The tube walls are made of acto-myosin and behave as an active viscoelastic material pumping the cytoplasmic fluid back and forth in a shuttle flow $[25,26]$. In response to environmental changes, the slime mold rapidly migrates toward attractive stimuli or away from repellents, efficiently turning the periodic shuttle flow into a net mass transport [38, $82,83,94]$. For P. polycephalum the dominant contraction frequency and its second harmonic make up the bulk of its oscillation modes which render it an ideal candidate to investigate the control of wave parameters on pumping performance in a living system [32].

In this letter, we investigate how a living system can control its waves' performance in a cost-efficient manner. Specifically, we study the performance of a peristaltic pump by evaluating the elastic deformation energy 

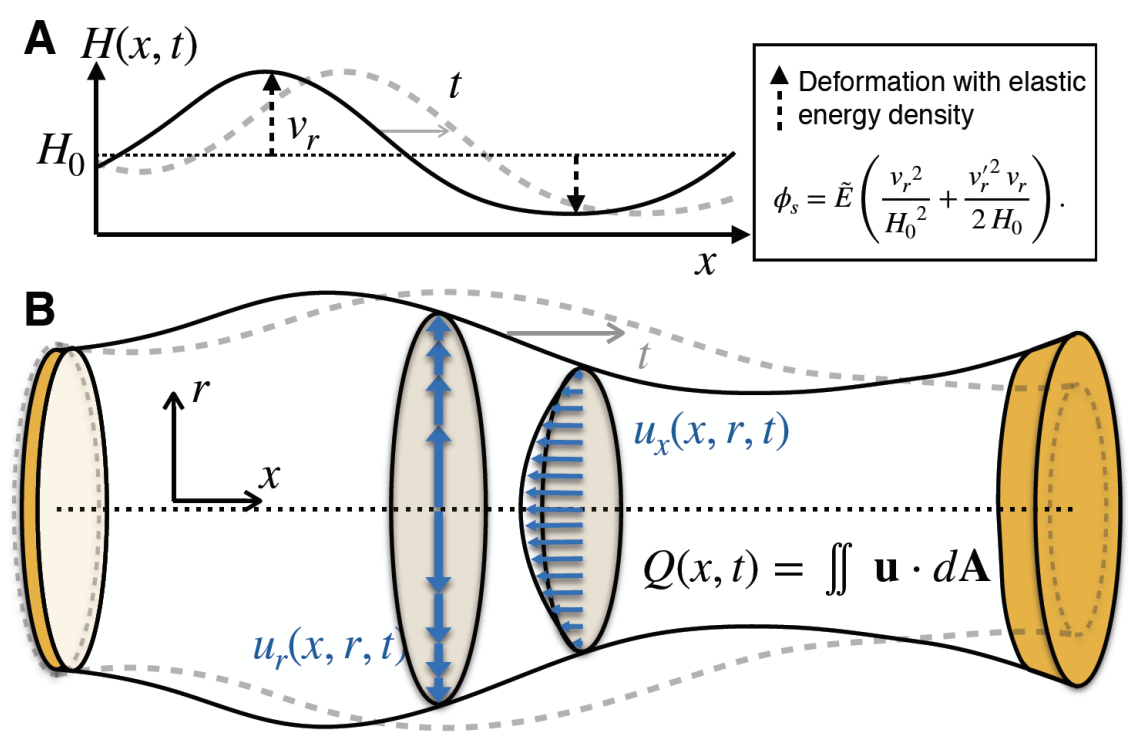

Figure 14: Scheme of tube as peristaltic pump and its elastic energy. (A) Deformation of the tube boundary $H(x, t)=H_{0}+v_{r}$ from the rest radius $H_{0}$ increases the elastic energy proportionally to the stretching energy density $\phi_{s}$ with a prefactor $\tilde{E}$. (B) Scheme of a tube acting as a peristaltic pump. The deformation of the tube boundary $H(x, t)$ over time drives radial, $u_{r}$, and axial, $u_{x}$, flow velocities and thus flow rate $Q(x, t)$ in the tube. 
of its contraction wave. The wave train consists of a dominant wave and its second harmonic introducing the phase difference between them as an additional adjustable parameter. We analytically show that the flow rate can be controlled by adjusting the phase difference while optimizing the required elastic energy for deformation. At the optimal phase difference the resulting wave shape occludes the tube most tightly, which optimizes pumping performance by over 25\% under physiological conditions. Investigating the peristaltic waves in P. polycephalum, we initially find that unforced specimen favour minimal occlusion. Only when forced to transport mass by a phototactic stimulus, specimens adjust their phase differences toward maximal occlusion optimal for pumping performance. Here, the phase difference serves as a subtle, but powerful parameter, in line with our theoretical predictions. To elucidate how the living system self-organizes its pumping performance we discuss non-linear tube wall visco-elasticity or active processes as a putative control for the phase difference adaption.

A peristaltic pump drives the directed transport of fluid through a circular tube by a periodic wave train of contractions $[68,69]$. We consider a single tube of shape $H(x, t)$ and length $L$ filled with an incompressible Newtonian fluid extending along longitudinal $x$ and radial $r$ coordinates, see Fig. 14 . In the limit of a long slender tube $L \gg H(x, t)$ the lubrication approximation applies [108], simplifying the equations governing the axial and radial flow velocity, $u_{x}(x, r, t)$ and $u_{r}(x, r, t)$ respectively, to

$$
\frac{\partial p}{\partial x}=\frac{\mu}{r} \frac{\partial}{\partial r}\left(r \frac{\partial u_{x}}{\partial r}\right), \quad \frac{1}{r} \frac{\partial\left(r u_{r}\right)}{\partial r}+\frac{\partial u_{x}}{\partial r}=0,
$$

where $p(x, t)$ denotes the pressure and $\mu$ the fluid's viscosity. Specifying a no-slip boundary condition at the wall then fully defines the flows as a function of pressure gradient $\frac{\partial p}{\partial x}$ and tube wall shape $H(x, t)$. The pumping performance solely due to the periodic wave train, i.e. without an applied pressure gradient, is then specified by the time-averaged volume flow rate $\bar{Q}_{0}$ over the wave period $T[68]$,

$$
\bar{Q}_{0}=\frac{2 \pi}{T} \int_{0}^{T} \frac{\int_{0}^{L} H^{-4}\left(x_{2}, t\right) \int_{0}^{x_{2}} \frac{\partial H^{2}\left(x_{1}, t\right)}{\partial t} \mathrm{~d} x_{1} \mathrm{~d} x_{2}}{\int_{0}^{L} H^{-4}(x, t) \mathrm{d} x} \mathrm{~d} t .
$$

To investigate how higher harmonics affect the pumping performance we assume the wall shape to be a superposition of two sinusoidal waves: a dominant wave and its second harmonic,

$$
H_{1+2}(\xi, \Delta \vartheta)=H_{0}+A_{1} \cos (2 \pi \xi)+A_{2} \cos (4 \pi \xi+\Delta \vartheta)
$$



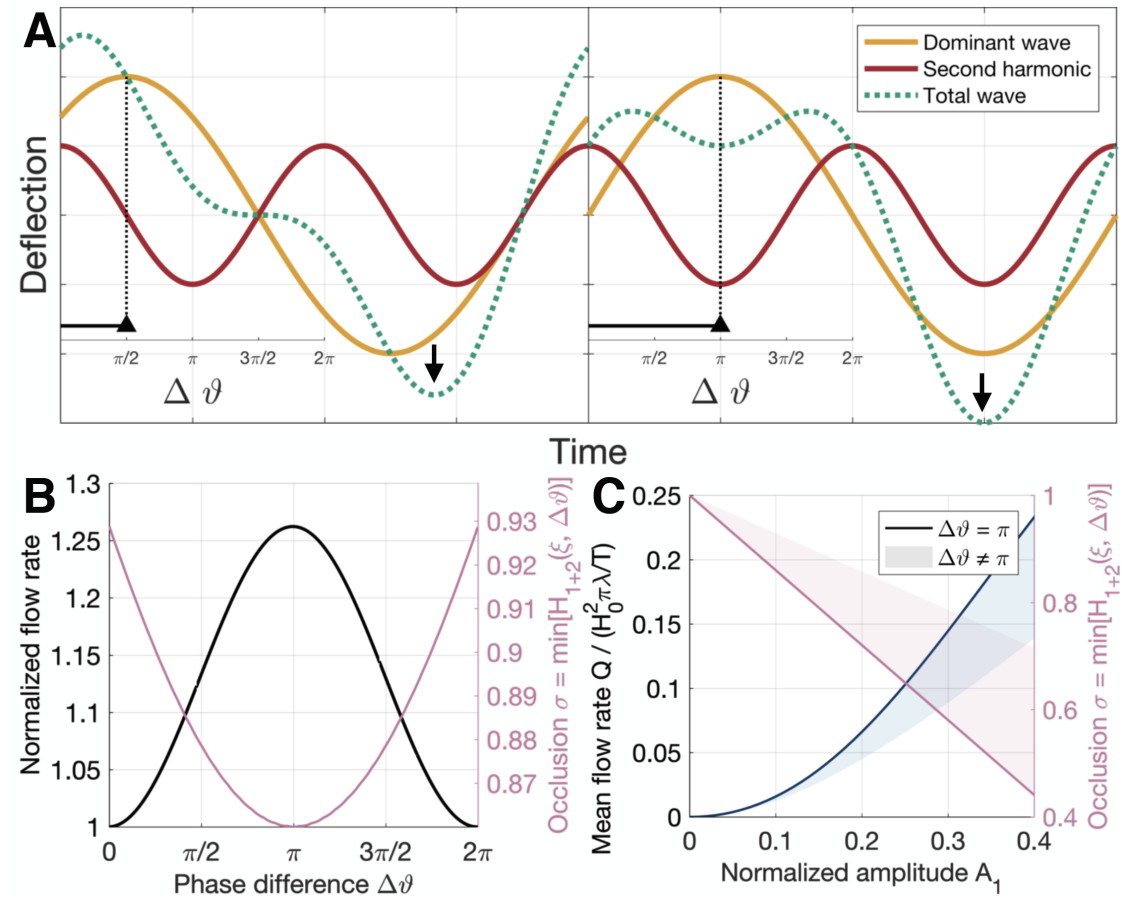

Figure 15: Phase difference $\Delta \vartheta$ between a dominant wave and its second harmonic controls flow rate by adjusting occlusion. (A) Scheme of dominant wave (yellow) and second harmonic (red) and their superposition (green dashed). Occlusion is maximal for $\Delta \vartheta=\pi$, where the minima of dominant wave and second harmonic align (right panel, compare arrows). (B) Flow rate and occlusion of $\mathrm{H}_{1+2}$ wave, Eq. Eq. (22), versus the phase difference shows maximal flow rate at $\Delta \vartheta=\pi$; amplitudes represent hysiological values for $P$. polycephalum $A_{1}=0.1 H_{0}$ and $A_{2}=0.04 H_{0}$ . (C) Dependence of flow rate (blue) and occlusion (pink) of $H_{1+2}$ wave on amplitude $A_{1}$ and phase difference (shaded area). Second harmonic amplitude is fixed at $A_{2}=0.4 A_{1}$ as above. Solid line highlights optimal condition at $\Delta \vartheta=\pi$. 
where $\xi(x, t)=x / \lambda-t / T$, with $\lambda$ the wavelength of the dominant wave, and $\Delta \vartheta$ the phase difference of the second harmonic with respect to the dominant wave, see Fig. 15A. Note, that the wavelength $\lambda$ and tube length $L$ can differ, but its effect is negligible on the pumping performance for $L \geq \lambda$ [68]. $H_{0}$ is the radius baseline around which the tube oscillates and $A_{1}$ and $A_{2}$ are the amplitudes of the dominant wave and its second harmonic, respectively. Furthermore, the tube's thin wall gets deformed from its baseline $H_{0}$ and stores a total elastic energy, $\bar{\Phi}$, of, see A.5.5 for derivation,

$$
\bar{\Phi}(H(x, t))=\frac{E h H_{0} \pi}{\left(1-v^{2}\right)} \int_{0}^{L}\left(\frac{v_{r}{ }^{2}}{H_{0}{ }^{2}}+v \frac{v_{r}^{\prime 2}}{H_{0}} v_{r}\right) \mathrm{d} x,
$$

with $v_{r}(x, t)=H(x, t)-H_{0}$ the radial deformation, $v$ Poisson's ratio, $h$ tube thickness and $E$ the Young's modulus. Note, that the contraction wavelength $\lambda$ is much larger than the tube's radius in P. polycephalum [28], which renders bending elastic energy along the axis negligible in comparison to radial deformation considered here, see A.5.5 for additional information. Therefore, the elastic energy per fundamental wave for the given wall shape $H_{1+2}$ and a Poisson's ratio of $\frac{1}{2}$ is

$$
\bar{\Phi}\left(H_{1+2}\right)=\frac{2 \pi}{3} E h\left(\frac{A_{1}^{2}}{H_{0}}+\frac{A_{2}^{2}}{H_{0}}+\frac{3 \pi^{2} A_{2} A_{1}^{2}}{\lambda^{2}} \cos (\Delta \vartheta)\right) .
$$

Following Eq. Eq. (24) the waves' amplitudes $A_{1}$ and $A_{2}$, dominate the elastic energy. The phase difference $\Delta \vartheta$ only contributes to higher order, scaling inversely with $\lambda^{2}$. For constant amplitudes the energy is minimized for $\Delta \vartheta=\pi$ and maximized for $\Delta \vartheta=(0,2 \pi)$, regardless of the wavelength of the contraction wave. Despite the negligible contribution to the elastic energy, varying the phase difference $\Delta \vartheta$ changes the waveform, see Fig. 15A, and most notably dramatically alters the resulting flow rate following Eq. Eq. (21), see Fig. 15B. Flow rate is maximal for $\Delta \vartheta=\pi$, and minimal for $\Delta \vartheta=(0,2 \pi)$. Considering physiological amplitudes of $A_{1}=0.1 H_{0}$ and $A_{2}=0.04 H_{0}$, see A.5.2, the phase difference shift can increase the flow rate by over $25 \%$. Hence, pumping performance of a peristaltic pump can be optimized with a cost-efficient shift towards a phase difference of $\Delta \vartheta=\pi$, thereby even minimizing elastic deformation energy. Note, that this result for a single tube can be extended to networks formed by P. polycephalum as peristaltic waves span the entire network with no dispersion at the vertices particularly in the networks selected for homogeneous architecture presented in the following, see A.5.4. 


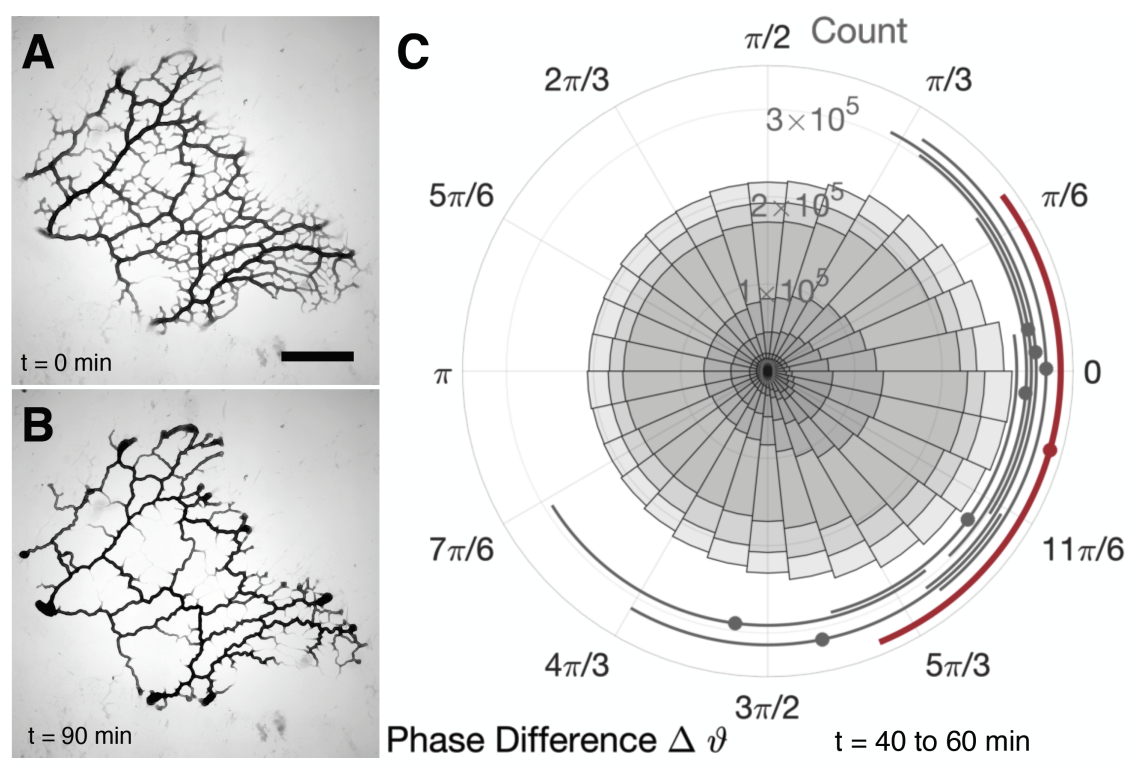

Figure 16: Unforced $P$. polycephalum has a phase difference distribution centered around $\Delta \vartheta=0$ corresponding to minimal occlusion. (A,B) Brightfield images of unforced P. polycephalum network $90 \mathrm{~min}$ apart. Bar $=2 \mathrm{~mm}$. (C) Stacked polar histogram of the phase difference $(n=7)$ between $40 \mathrm{~min}$ to $60 \mathrm{~min}$ after start of data acquisition. Dots indicate mean and lines $33 \%$ intervals. In red the mean and interval for the total distribution.

We identify the tighter occlusion of the tube, i.e. the alignment of the minima of the first and the second harmonic for $\Delta \vartheta=\pi$, as the reason for the increased flow rate, see Fig. 15B,C. To gain intuition why a tighter occlusion increases the flow rate one can interpret the flow rate in Eq. Eq. (21) as a weighted average with the weight given by $H^{-4}(x, t)$. Even though the weighted term is periodic in space and time, the total weight is increased for small $H(x, t)$,i.e. tighter occlusion, which increases the average flow rate maximized at $\Delta \vartheta=\pi$.

Having derived in theory how big the effect of a shift in phase difference between dominant wave and second harmonic can be, we turn to quantify this defining wave parameter in a living system. Our model system here is P. polycephalum, an organism gaining attention as a living network-shaped peristaltic pump. In fact, all individual tubes of the network undergo peristaltic contraction.

P. polycephalum (Carolina Biological Supply Company) networks were prepared with an oat flake or microplasmodia culture on $1.5 \%$ agar plates [17, 75]. Networks were re-cultured every three days for oat flake culture. After an overnight culture, the networks were selected for homogeneous tube di- 
ameter and segment length and then cut to a size of roughly $30 \mathrm{~mm}^{2}$ with an inoculation loop $90 \mathrm{~min}$ before data acquisition with 3 to 6 seconds per frame to mitigate any reaction from cutting [75]. Peristaltic contraction dynamics throughout the network are quantified from brightfield images with a custom written Matlab program [75]. To quantify the phase difference $\Delta \vartheta$ between the second harmonic and the dominant wave in the network-wide contractions we first map out each tube's contractions. The contractions' traces over time are high-pass filtered and band-pass filtered for the dominant and the second harmonic, respectively. The phases of the two waves are isolated with a step-wise fitting of the corresponding waveform to the filtered data, see A.5.1 for an in-depth description.

Being inspired by Purcell's observation [110] that living organisms may only optimize their most pressing problem we next force P. polycephalum to transport mass by providing a phototactic stimulus. P. polycephalum's phototactic response is wavelength dependent, with the strongest avoidance reaction to blue light at around $450 \mathrm{~nm}$ [59]. We applied blue light with a halogen lamp (Illuminator HXP 200C) and a filter set (Zeiss $38 \mathrm{HE}$, excitation BP 470/40) continuously from light onset, designed to trigger mass transport away from the networks' center. Illuminated regions were fully surrounded by network and are chosen to have a size of roughly $30 \%$ to $50 \%$ of the total network size at the start of recording. Image recording started 60 min before light exposure allowing us to track phase shift evolution over time before complete relocation from the illuminated region, see Fig. $17 \mathrm{~A}$, B. We bin the distribution of phase differences $\Delta \vartheta$ into time windows of $20 \mathrm{~min}$, once right before the onset of illumination and two sets after onset of illumination, see Fig. 17 C, D, E. Confirming our previous observations of unforced specimen the phase difference distributions before the onset of illumination are peaked around $\Delta \vartheta=0$. Yet, forcing the specimen with light to increase mass transport we now observe that under blue light illumination the phase difference successively shifts toward $\Delta \vartheta=\pi$, the optimal phase difference for peristaltic pumping, see also A.5.3. Why is the second harmonic aligned inefficiently around $\Delta \vartheta=0$ when unforced? And does the living system actively adjust the second harmonic of its peristaltic wave for higher pumping performance?

Our experimental approach allows us to quantify the tube deformation, but not if the deformations' second harmonic is arising actively or passively. Therefore, from data we cannot infer if the surprising adjustment of phase difference following forcing is an active adaptation of contrac- 


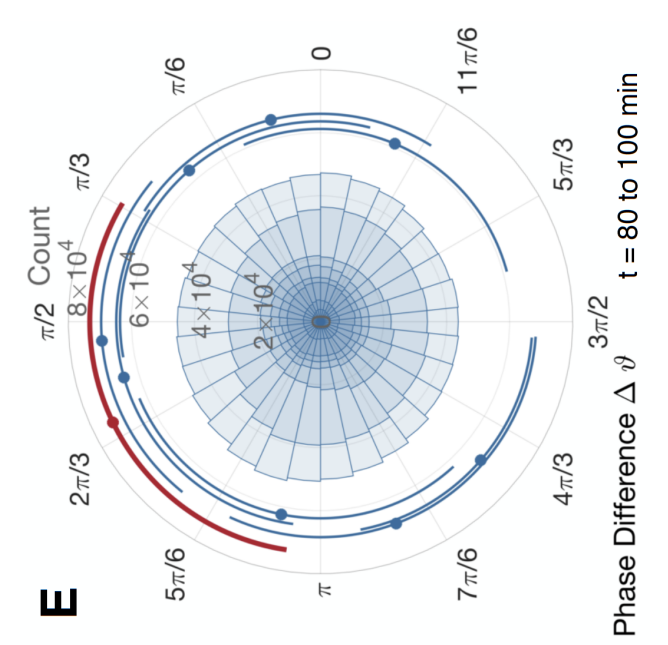

近国苛 㐫 氖㐫 छ $\dot{\Xi}$

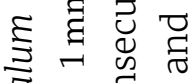
व) ญ्ष๊ \&

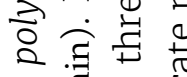

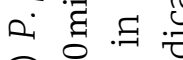

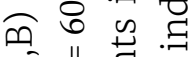
$\varangle+$ 过

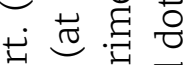

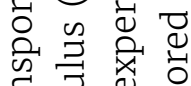
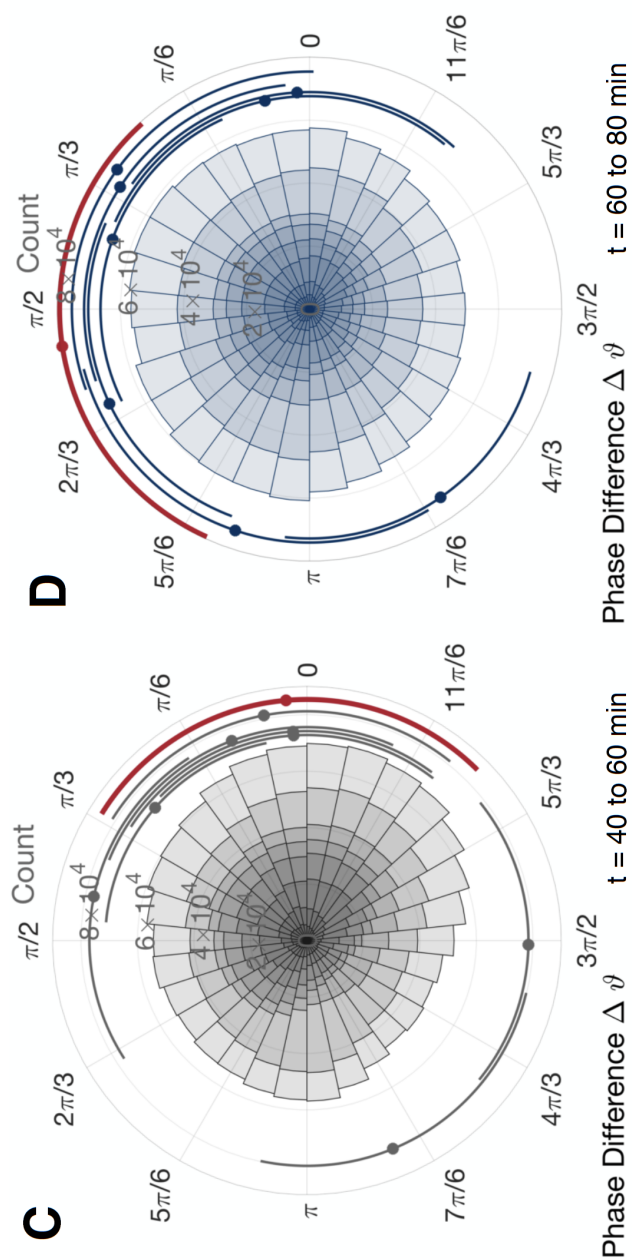

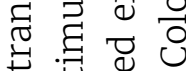
is is

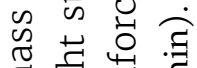
范范 \& 胥

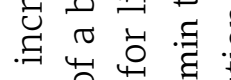
운 पु

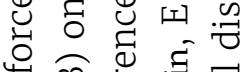

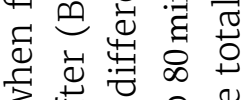
了芯过

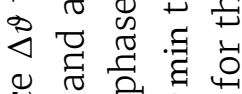

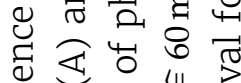
¿ ¿

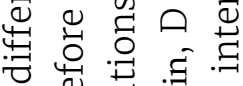
ه

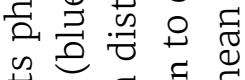
.

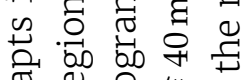

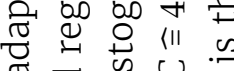

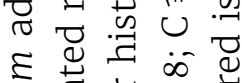
竞泀

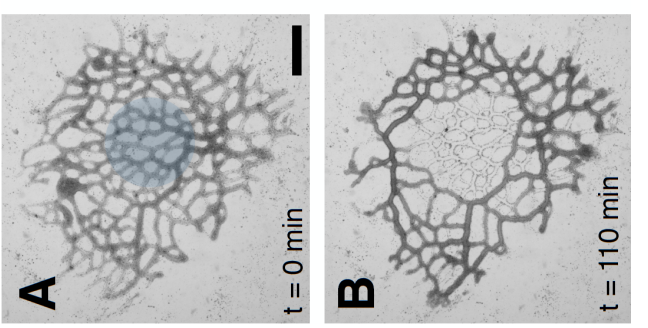
Qิ

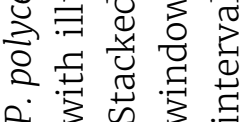
$\ddot{\bar{N}}$

r

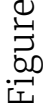


tions of the living system. It is, however, instructive to compare our observations to the wave shape forming in a visco-elastic tube filled with a viscous fluid driven solely by a dominant wave $F_{P} \sim \cos (2 \pi \xi)$. The tube responds with a second harmonic in deformation arising passively: Following the approach of Takagi and Balmforth [111] and assuming a weakly visco-elastic restoring force $F_{E}=\frac{E h}{\left(1-v^{2}\right) H_{0}^{2}} v_{r}+\eta \frac{\partial v_{r}}{\partial t}$, with dampening coefficient $\eta$, we expand systematically for small $v_{r}$ as in [112] to derive dominant and second harmonic of the tube deformation, see A.5.6. Their phase difference is $\Delta \vartheta=\frac{\pi}{2}-\arctan \left(\frac{4\left(1-v^{2}\right)}{E h T}\left(\frac{\mu \lambda^{2}}{\pi H_{0}}+\pi \eta H_{0}{ }^{2}\right)\right)$ and is constraint between $\Delta \vartheta \in\left[0, \frac{\pi}{2}\right]$. For physiological parameters (see A.5.2), $\Delta \vartheta \sim 0.1$ close to the observed minimal occlusion in unforced networks. Thus, the non-optimal $\Delta \vartheta \sim 0$ in unforced data is in agreement with a fluid-filled visco-elastic tube with fundamental forcing only. Furthermore, a phase difference outside the range $\left[0, \frac{\pi}{2}\right]$ can result only from non-linear material behaviour or active forces. Strain-hardening would drive the phase difference toward $\pi$, in line with our observations for forced specimen, see A.5.6. Even though geometric non-linear effects remain small, an adaptive non-linear material property may be a convoluted, yet surprisingly efficient way to adapt the phase difference and therewith pumping efficiency. Alternatively the slime mold may actively control its phase difference, i.e. by utilizing two separate oscillators with an adaptive phase relation. The high, and surprisingly stable, amplitude ratio $A_{2} / A_{1}$ is indicative for the latter, with the passively arising overtone acting as a reference point when unforced.

Investigating how living systems can control their wave's performance in a cost efficient manner we here identified the phase-difference between wave harmonics as a subtle but powerful parameter. We find that the performance of peristaltic pumping can be enhanced by over $25 \%$ while lowering the elastic energy cost by optimizing the phase difference between the dominant wave and its second harmonic of the contraction wave. Testing for this mechanism in the slime mold $P$. polycephalum, we observe a shift in phase difference toward optimal pumping performance when forced to transport mass in response to environmental changes. We identify a passive visco-elastic response as a putative source for the overtone and find that non-linear material behaviour can adapt the phase difference efficiently.

Our findings spotlight the important role of higher harmonics in wave phenomena. Higher harmonics intrinsically arise in numerous systems like coupled oscillators [5] or active visco-elastic materials [111, 113]. Living 
systems, as exemplified here, use the phase difference between harmonics as a key cost efficient parameter to adjust wave performance. Specifically, control may reside in the inherent non-linear material properties that may change upon environmental stimuli [37]. It is inspiring to speculate that life's bio-chemical machinery can quickly adjust its visco-elastic properties of an actin-cortex, e.g. by controlling the access to globular actin via secondary proteins [24]. A control that could prove powerful to synthetic biology. In conclusion, our findings provide a novel metric for wave phenomena in general, namely the phase difference between wave harmonics. We demonstrate the crucial role and control residing in non-linearities of living systems. Our life-inspired findings on flow control in peristaltic pumping may find direct application in the design of smart materials and soft robotics. 

Part IV

DISCUSSION AND OUTLOOK 

The dynamic morphology in Physarum polycephalum networks is the main measure for its puzzling 'intelligent' behaviour. The slime mold needs to transport mass from pruning tubes towards more favourable regions and excels at the task in a self-organized - and baffling - manner. With this work I present how Physarum polycephalum organizes its mass transport for two complementary stimuli: a wound healing response and the photoavoidance to blue light.

Crucially for both studies I present a comprehensive image analysis to map contraction dynamics in space and time. Therewith I surprisingly found a cut to be an attractive stimulus, with the contraction response showing a multi-step pattern until the wound is healed. Furthermore, a central and fully severing cut induces a cessation of contractions stalling flows within the network for a short duration. In blue light stimulated networks I identified the phase-difference between contraction harmonics as a subtle but powerful control parameter for pumping efficiency. Measuring it, I found the slime mold to align its contractions to maximize the occlusion of tubes, in turn increasing the peristaltic pumping efficiency. I here want to discuss the relevance of these findings and highlight where this research may go from here on.

While peristalsis does facilitate mass transport in Physarum polycephalum what role do other pumping mechanisms play? The necessary traveling wave is prevalent in Physarum polycephalum networks ${ }^{28}$, but standing or spiral waves also exist ${ }^{114}$, see Figure M4. Furthermore, the cessation of oscillations in wounding is already accompanied with a growth towards the cut site; some network-forming slime molds even migrate while completely lacking the contraction oscillation ${ }^{115}$. Therefore, it seems likely that local non-oscillating contractions cause a pressure gradient which cause a steady flow of mass. Yet, in peristalsis, with physiological amplitudes and a phase difference around $\Delta \varphi=\pi$, the flow rates can be as high as $\hat{\bar{Q}} / V=0.18$, with $V$ the total volume of a tube. In a setting where a tube connects two unconstrained reservoirs and pumps with a traveling wave 
the tube can prune its own volume in $10 \mathrm{~min}$ to $15 \mathrm{~min}$. Under blue light or after a cut Physarum polycephalum usually prunes or pumps for a minimum of 20 min agreeing on the time scales. It is inspiring to speculate that nonoscillating contractions may be a precursor giving a cue to the network to build up up a coordinated and efficient peristaltic pattern for a collective response. Regions can differentiate themselves behaviourally to build up a directed mass transport in time, in line with the multi-step pattern in wounding and the phase alignment in blue light experiments. Here, exploring the similarities and differences between stimuli can isolate either pumping mechanism and highlight the interplay of them.

Given the insights and the understanding of mass transport, how do we explain the puzzling 'intelligence' in Physarum polycephalum? The immediate step at hand is building a self-organized model that combines attractive and repulsive stimuli, a crucial aspect for the adaptability in Physarum polycephalum. The question arises which parameter can guide the direction of mass transport, but can also be influenced by various stimuli. The results present viscoelasticity as a promising candidate: after a cut the exposed endoplasm turns slowly into fan undergoing a sol-gel transition, the overtones in blue light experiments are related to non-linearities in the mechanical properties and the ectoplasm contracts rapidly under blue light indicating bundle formation (see Figure A1). At the same time the local elasticity or viscosity can be easily controlled biochemically and thereby influenced by a variety of stimuli. For example, with the actin-actinin mix in the cytoplasm the slime mold may act on the percolation threshold of actin formation ${ }^{116}$, easily forming or dissociating actin filaments to change its mechanical properties, see Section 2.1.1. Together with flow dependent growth ${ }^{78}$ and a stimuli dependent active actomyosin, a contraction relaxation cycle model ${ }^{37}$ may describe growth, adaption, network formation and self-organization from local rules; all features which are prominent in Physarum polycephalum and in combination display the puzzling 'intelligence'. Estimated contractions can be directly compared to the presented results, e.g. the phase-difference, the frequency adaption or the response pattern to a severed connection, to validate the model and estimating the adaption in the mechanical parameters. Such a model may prove to solve complex tasks like the traveling salesmen problem efficiently and offers new computation algorithms stemming directly from active hydrodynamics. 
Experimentally it is paramount to isolate key behavioural components and link them to their molecular counterparts. As a single cell that still shows behaviour Physarum polycephalum can be an ideal candidate to study receptors and their downstream effects. And the slime mold field is closing in on a milestone to achieve that: the genome has been sequenced recently ${ }^{13}$ and transfection or gene editing tools like CRISPR/Cas9 can open up a whole plethora of discoveries. Nuclei can be labeled for easy and noninvasive flow measurements making large-scale comparison of flows and contractions possible. Photoreceptors can be isolated for identification to e.g. test if overtones arise from a single pathway or from an interplay of photoreceptors. Furthermore, cytoskeletal components can be labeled to pinpoint mechanical changes or include optogenetics to invoke a targeted stimuli response. Its single-celled nature easily up scales a single amoebal gene edit into a plasmodium whose behaviour can be readily quantified. A whole new set of phenotypes can be created where the interplay of essential molecular components and behaviour leads the way to exploring comprehensive mechanisms, substantiating Physarum polycephalum as a model organism.

Taking it a step further, can Physarum polycephalum also directly be useful in future applications? The coordinated mass transport combined with an easily controllable specimen and a variety of receptors may make slime molds an asset for targeted collection. Slime molds have an inherently high resistance to the toxicity - and surprisingly an affinity - to heavy metals ${ }^{117}$ and may be genetically engineered to clean up environmental pollutants. Preliminary experiments in our laboratory and elsewhere ${ }^{118}$ show that they readily incorporate metals and accumulate them within their cytoplasm. Furthermore, as slime molds naturally feed on bacteria by phagocytosis, a transformation to target a specific pathogen or biological waste is easily conceivable. Combined with the rapid growth rate, vast size, comparatively fast taxis and the easy handling of slime mold cultures, they might remould safe pollution handling.

Combined the theoretical advances, the experimental options and the versatility of Physarum polycephalum are mutually beneficial fostering a research field which transcends into various related fields of research and development. Fluid flow coupled to a mechanical feedback are inherent in vascular systems and crucial for the developmental stages of blood vessels, leaf venation or fungal networks. Auto-oscillatory spatial patterns, 
including spiral and traveling waves, are a prime matter of research for epilepsy in the brain and for cardiac arrest in the heart. Photo-, mechano, chemo- and hydro-taxis are all prominently present in Physarum polycephalum with receptors that share domains with various other clades and are essential for every life form to prosper. Pumping mechanisms - and in particular peristalsis - are found in most living systems, but also in numerous technological applications and recently gain increased attention in soft robotics.

Last but not least Physarum polycephalum already found its way to reconsider what we perceive as intelligent. With its unique interplay between local rules and global behaviour it provides a principle understanding of how problem solving in a self-aware and adaptive system can function. On the one hand this helps to improve artificially created intelligent systems or to possibly conceive a hydro-mechanical computation method in contrast to the electromagnetic basis we use in our everyday life. And on the other hand the 'intelligent' slime mold might reach as far as teaching us a lesson in philosophy: If a single cell can memorize, adapt, decide and respond at which point does it start to think? 
Part V

APPENDIX 



\section{A.1 Material list}

The following materials were used in the experimental setups, either for slime mold culture or for microscope setups. Refer to the list for product detail and subsequent protocols for media. In the following, ${ }^{\dagger}$ always refers to an item from this material section for quick look-up.

\section{Agar plates}

If not stated otherwise all plates were prepared with 1.5\% Agar powder for bacteriology and ddW, VWR International LLC.,Art.Nr.: 20767.298, with 100x15 mm petri dishes, Falcon Corning, VWR International LLC., Art.Nr.: 391-2002

Calcium chloride dihydrate

Calcium chloride dihydrate $\geq 99 \%$, p.a., ACS Reagent for molecular biology, Carl-Roth GmbH\&Co KG,

Art.Nr.: 5239.2

Citric acid monohydrate

Citric acid monohydrate $\geq 99.5 \%$, Ph.Eur., Carl-Roth GmbH\&Co KG, Art.Nr.:5110.3

$D(+)$-glucose anhydrous

D(+)-Glucose Reag. Ph. Eur., VWR International LLC., Art.Nr.: 1.08337 .0250

Dipotassium phosphate

di-Potassium hydrogen phosphate trihydrate $\geq 99 \%$, p.a formula $\mathrm{K}_{2} \mathrm{HPO}_{4} \cdot 3 \mathrm{H} 2 \mathrm{O}$, Carl-Roth GmbH\&Co KG, Art.Nr.: 6878.2

Disodium phosphate

di-Natriumhydrogenphosphat-Dihydrat $\geq 99.5 \%$, VWR International LLC., Art.Nr.: 1.06580.0500 
EDTA

Ethylenediamine tetraacetic acid disodium salt dihydrate, CarlRoth GmbH\&Co KG, Art.Nr.: X986.1

Formaldehyde-solution

Formaldehyde solution 37\%, Carl-Roth GmbH\&Co KG,Art.Nr.: 4979.1

Glycerol

Electran Molecular biologoy grade Glycerol, VWR International LLC., Art.Nr.: 444482V

Hemin

Purity $\geq 98 \%$, HPLC grade, Alfa Aesar, Art.Nr.: A11165

Iron(II) chloride tetrahydrate

Iron(II) chloride tetrahydrate, Sigma-Aldrich Inc., Art.Nr.: 1038610250

Magnesium sulfate heptahydrate

Magnesium sulfate heptahydrate puriss. p.a., ACS reagent, $\geq 99.0 \%$ (KT), Sigma-Aldrich Inc., Art.Nr.: 63140

Oat Flakes

Echte Kölln Kernige

Difco-Bacto-Soytone

Sojamehl-Pepton (Bacto-Soytone), Otto Nordwald GmbH, Art.Nr.: 243610

Physarum sclerotium

Physarum Sclerotium, Carolina, Art.Nr.: 156190

Potassium dihydrogen phosphate

Potassium dihydrogen phosphate $\geq 99 \%$, p.a., ACS Reagent for phosphate, Carl-Roth GmbH\&Co KG, Art.Nr.: 3904.2

Sodium chloride

Natriumchlorid $\geq 99 \%$, Ph.Eur., USP, Carl-Roth GmbH\&Co KG, Art.Nr.: 9265.1

Yeast extract

Yeast Extract for use in microbial growth medium, 250g, SigmaAldrich Inc., Art.Nr.: Y1625 


\section{Bacto-tryptone}

BD Bacto ${ }^{\mathrm{TM}}$ Tryptone, BD Biosciences, VWR International LLC., Art.Nr.: 90000-286

\section{A.2 Agar overlay light stimulus}
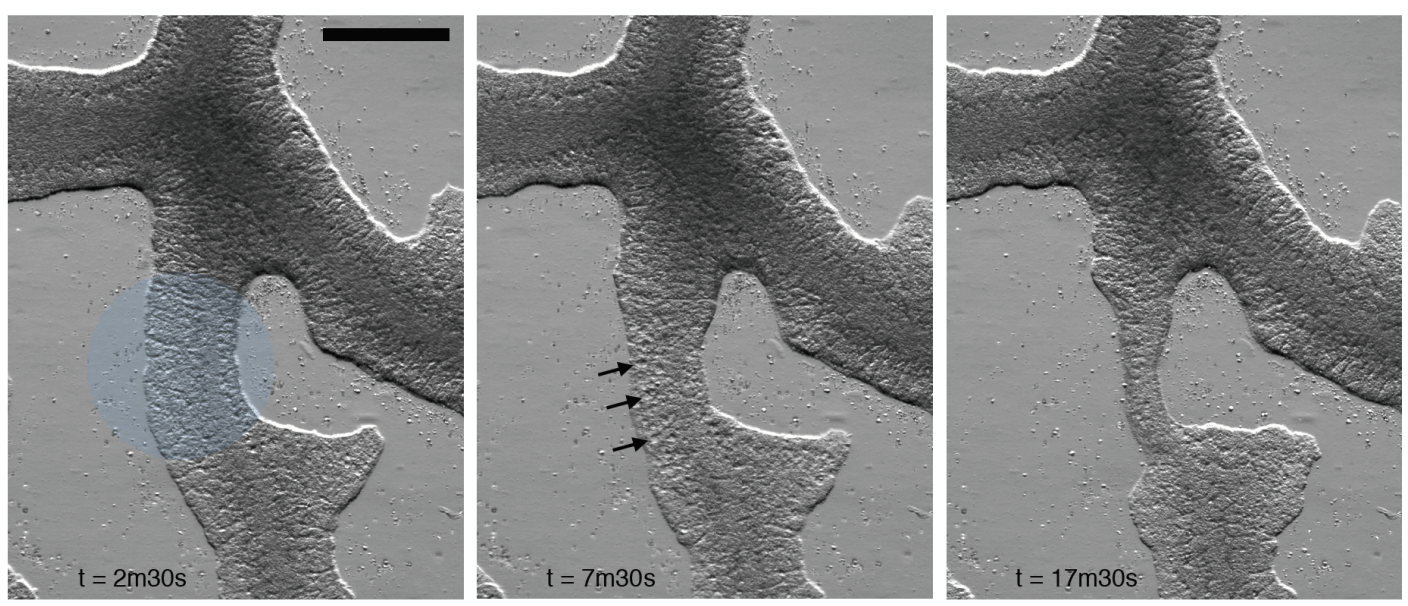

Figure A1: Blue light stimulus on agar-overlay experiment. Squished tubes are made by putting a $2 \mathrm{~mm}$ thick agar layer above microplasmodia and let them grow overnight. Blue region in left most image was illuminated for $18 \mathrm{~min}$. Flow in the exposed tube ceases shortly after stimulus onset and turns into an outward flow only. Vacuoles form (arrows) after some time being advected with the outward flow. Scale bar $=200 \mu \mathrm{m}$

\section{A.3 Hilbert transformation}

This supplemental information is based on $^{119}$. To obtain the quantitative key parameters namely amplitude $A$, frequency $f$ and phase $\varphi$ of contractions, we use the Hilbert transformation.

The Hilbert transform $h(t)$ of a function $a(t)$ is defined as:

$$
h(t)=\frac{1}{\pi} \int_{\infty}^{\infty} \mathrm{d} \tau \frac{a(\tau)}{t-\tau}
$$

where $h(t)$ is phase shifted by $-90^{\circ}$ for all positive frequencies and by $90^{\circ}$ for all negative frequencies. With the real function $a(t)$ and its Hilbert transform $h(t)$ one can form the complex analytical signal

$$
c(t)=a(t)+i h(t)
$$


which can be used to derive the instantaneous amplitude

$$
A(t)=|c(t)|
$$

and phase

$$
\varphi(t)=\arg (c(t)) .
$$

The frequency is given by differentiating the phase $f=\dot{\varphi}$.

For the presented results each data point (skeleton pixel) is treated as a discrete time series which can be Hilbert transformed accordingly into an analytical signal. To approximate the analytic signal from discrete data, we calculate the fast-fourier transform (FFT) of the input sequence, replace those FFT coefficients that correspond to negative frequencies with zeros, and calculate the inverse FFT of the result [120].

The obtained key parameters are stored as a set for each pixel, i.e. [a(t), $A(t), \varphi(t), f(t)]_{k}$ where $k$ represents all pixels in space.

\section{A.4 Kymograph based analysis}

As described in section 2.1 the control of plasmodia network parameters like fan amount, motility, size or grid density is limited mostly to selection of parallel culturing. Therefore quantitative analysis as described in 2.2 is not suitable for every experiment. To analyse the bulk of experiments we use a kymograph based approach described in the following.

A kymograph is a 3D representation of designated data with one space coordinate, one time coordinate and one value (intensity) coordinate. In our case space is represented as line through a main vein in a network (compare with E1, red line). Along this line a 3px orthogonal average of the light intensity is taken for the value coordinate. This is done for every image and concatenated in time.

Along the time coordinate the Hilbert transform is performed for every point in space (see A.3). Here, additionally, the amplitude is computed with a root-mean-square moving average (RMS-MA) filter besides the absolute value of the analytical signal. After computation the results are averaged in space and plotted in a line plot to depict contraction dynamics. 
A.5 supplemental material for 'living system adapts harmonics of peristaltic wave fo

A.5 Supplemental material for 'Living system adapts harmonics of peristaltic wave for cost-efficient optimization of pumping performance'

This is the supplemental material for Chapter 6 and is presented as submitted to the journal. Therefore parts of the methods are repeated in short, in particular A.5.1.

\section{a.5.1 Phase difference determination}

The phase difference between the dominant wave (first harmonic) and its second harmonic of the contraction pattern proves to be a crucial parameter to alter the pumping performance of a peristaltic pump. In the following we want to present how we determine the phase difference from Physarum polycephalum image data.

Initially the time series of bright field images is segmented into network and background. This binary image is skeletonized to represent the network structure and lastly stored in a reduced, pixel-resolved manner to represent the contraction dynamics. This is described elsewhere in more detail [75] . The important quantity we obtain is the light intensity and the diameter data resolved in space and time. They anti correlate as a thicker tube blocks more of the transmission light according to Beer-Lambert law. From here on we use calibrated intensity data to make use of its higher resolution (dynamic range versus pixel resolution).

The obtained data is prone to temporal and spatial noise. To reduce the spatial noise, the intensity is smoothed in a $30 \mathrm{px}$ local disk with a Gaussian. Phase patterns usually occur on larger length scales [28]. Furthermore, we high-pass filter the data, omitting growth and pruning to isolate the contraction pattern. For the high-pass filter and the later used band-pass filters we use Kaiser windows as the tapering function and orders between $n=50$ to 150 .

With the Hilbert transform we determine the primary frequency $f_{P}$ of the contraction pattern for the whole data set. The frequency does change over the course of an $3 \mathrm{~h}$ experiment - and crucially has to for phase adaptation to happen -, yet the changes are small and happen within the chosen bandpass filter. Fig. A2 shows the frequency spectra of each forced experiment, which shows that while the frequency distributions are fairly broad the 
bulk of the energy is made up of the dominant and second harmonic frequencies. A data set specific primary frequency $f_{P}$ is used as the baseline to band-pass filter the first $f_{1}=f_{P}$ and second harmonic $f_{2}=2 f_{P}$. In this way, for every point in space, the time series is split into the respective first and second harmonic.

Each harmonic is fitted in a step-wise manner to a fitting function $F_{1 / 2}(t, \varphi)=$ $\sin \left(2 \pi f_{1 / 2} t+\varphi\right)$, with $f_{1 / 2}$ the frequency of the first or second harmonic, $t$ the time and $\varphi$ the phase. The step width is chosen as two periods of the main frequency. Furthermore, the data is divided by its amplitude and then fitted to the fit function determining the phase $\varphi$ with the Matlab function Isqnonlin.

The resulting phases now give the phase difference $\Delta \vartheta=\varphi_{2}-2 \varphi_{1}$ in the reference system of the second harmonic. The fit residuals and local amplitude can be used as indicators for goodness of the estimation. For example high residuals often indicate a local phase jump or low amplitudes in the second harmonic compared to the first harmonic indicate a poor signal-to-noise ratio.

\section{a.5.2 Physiological parameters}

Peristaltic waves in P. polycephalum can be characterized by: tube radius

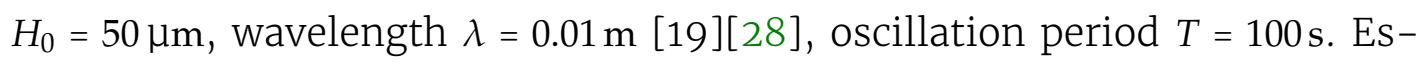
timates for physiological parameters of tube wall and cytoplasm mechanical properties from $P$. polycephalum or related organisms are: cytoplasm viscosity $\mu=6.4 \times 10^{-3} \mathrm{Pas}[72]$, tube wall effective stiffness $K=\frac{E h}{\left(1-\nu^{2}\right) H_{0}{ }^{2}}=$ $2.7 \times 10^{5} \mathrm{~Pa} \mathrm{~m}^{-1}$ (assuming a Young's modulus of $100 \mathrm{~Pa}[109,121]$ and tube wall thickness of $h=H_{0} / 10$ ), tube wall effective viscosity $\eta=K \times 100$ s [113]. For $\kappa$, using the above parameter estimates, we arrive at $\kappa=\frac{4 \mu \lambda^{2}}{\pi H_{0}^{3} K T} \approx 0.2$.

Besides the phase difference, the amplitudes of contractions are a defining factor for the pumping performance of a peristaltic pump. It is reported that blue light may induce a transient increase in amplitude shortly after the onset of light [61]. This is in line with our experiments and we, furthermore, see a transient decrease in amplitude roughly 10 min after the stimulus onset, see Fig. A3. Yet crucially, the ratio of the dominant to the second harmonic amplitude stays constant for $30 \mathrm{~min}$ after the onset of light. So while the pumping performance does alter with the amplitude, the increase of pumping performance due to the alignment of the phase 


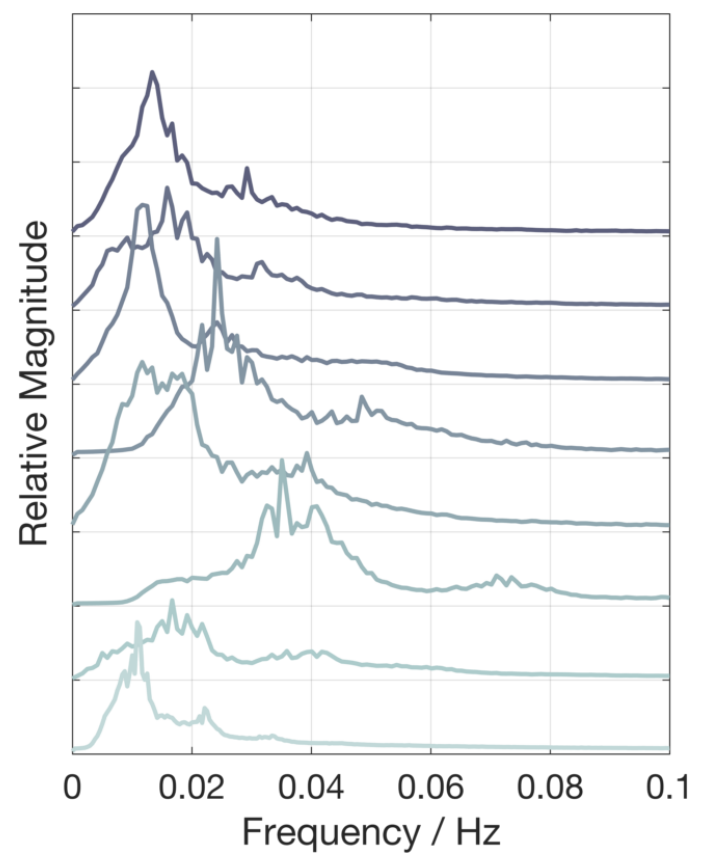

Figure A2: Frequency spectrum in the window of interest ( $40 \mathrm{~min}$ to $100 \mathrm{~min}$ ) of the 8 stimulated experiments. Relative magnitude is plotted for each experiment over the respective frequencies and are shifted with respect to each other on the $\mathrm{y}$-axis. To compare to figure A4 experiment numbers are descending from top to bottom. Mind that the dominant and second harmonic frequency make up the bulk of the energy, but do vary within their distribution.

difference remains unaffected. At later stages the pruning of veins and growth of foraging fans renders the data more prone to noise or artifacts, hence the increase in amplitude ratio should be seen with care. 

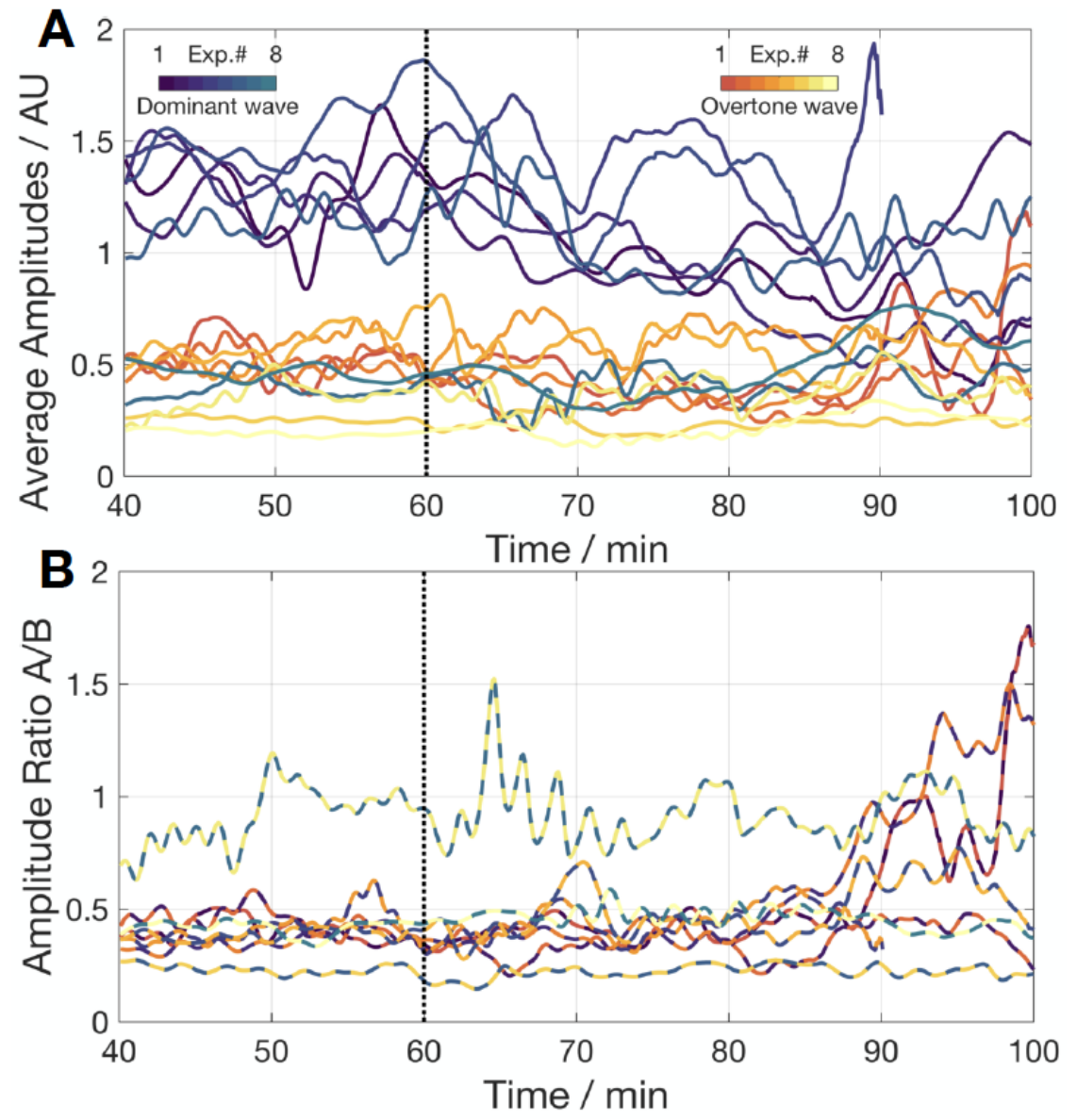

Figure A3: Amplitudes of contractions may adapt over time, while the ratio of dominant to second harmonic amplitude stays constant after a blue light stimulus. (A) Spatial average of the contraction amplitude of 8 experiments of the dominant (blue-teal gradient) and overtone (redyellow gradient) amplitudes. Amplitudes can transiently increase after the onset of light (60 min and drop roughly at $70 \mathrm{~min}$. (B) The ratio of amplitudes dominant/overtone(second harmonic) shown in (A). The ratios stay constant over time despite onset of a blue light stimulus, expect for one experiment (blue/yellow). They diverge at later times due to an increase in noise and artifacts as the network morphology changes dramatically. (A) shows that the blue/yellow outlier has a strongly reduced dominant amplitude, which suggest a reduced activity in the specimen. 


\section{a.5.3 Time development of experiments}

In the main text we describe the progression of the phase difference distributions $\Delta \vartheta$ over time and in particular show that the total distribution moves towards $\Delta \vartheta=\pi$. Taking a closer look at the progression of the mean values for each individual experiment, see Fig. A4, we can also show that 6 out of 8 experiments move towards higher occlusion individually when subjected to blue light. Only 2 experiments (number 1 and 5) do not, or only partly move towards higher occlusion. Yet, the majority makes this progression towards higher occlusion over the positive flank along the unit circle, i.e. firstly towards $\Delta \vartheta=\pi / 2$ and then towards $\Delta \vartheta=\pi$. Progression differs in the time windows, some experiments show early progression, i.e. within the first $20 \mathrm{~min}$, and some experiments progress only $20 \mathrm{~min}$ later in the second window.

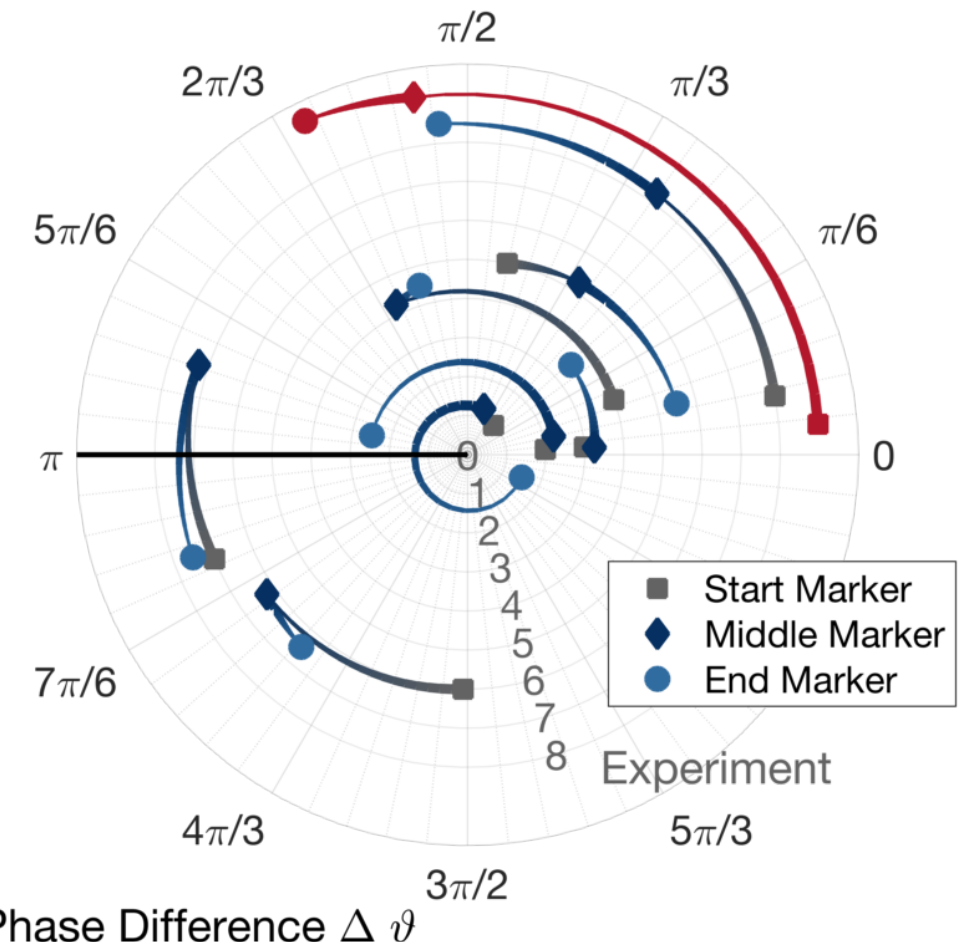

Figure A4: Phase difference aligns towards $\Delta \vartheta=\pi$ after light stimulus forces network to transport mass. Each marker is the mean value in phase difference of a $20 \mathrm{~min}$ time window: the start marker (grey) from $40 \mathrm{~min}$ to $60 \mathrm{~min}$, the middle marker (dark blue) from $60 \mathrm{~min}$ to $80 \mathrm{~min}$ and the end marker from $80 \mathrm{~min}$ to $100 \mathrm{~min}$. In red is the mean of the total distribution and the bold line at $\Delta \vartheta=\pi$ shows the point of maximal occlusion. 


\section{a.5.4 Spatial wave patterns}

In our theoretical consideration of a peristaltic pump and its energy cost we consider a single tube. Yet, it is evident that Physarum polycephalum is an interconnected network of tubes and the effect of network architecture has to be scrutinized. In order to extrapolate the findings on pumping performance from a tube to a network its contraction patterns have to be coordinated over the total network extend. It has been recorded before that peristaltic waves in Physarum polycephalum span the whole system size and critically show no dispersion [28]. Hence, we have to measure the spatial coordination of the dominant and second harmonic contractions in our experiments to validate the single tube approximation for the whole network.

As measuring a spatial wavelength is ambiguous in interconnected networks we chose to display the phase patterns in time and space to illustrate peristaltic patterns in our experiments for two exemplary data sets, see Fig. A5. Along the spatial axis the data points are sorted depending on the euclidean distance to the center of illumination, hence illuminated regions are close to the ordinate axis, but adjacent values do not have to be in the same spatial neighbourhood. The color indicates the respective phase, of either dominant or overtone contraction, with the fixed contraction frequency removed. In this visualisation a single traveling wave can be seen as a slightly tilted horizontal line of constant phase.

Both data sets show primarily system spanning waves during the time of interest or at least phase patterns on scales much larger than a single tube which in both experiments are on the order of $\sim \frac{1}{20}$ th to $\sim \frac{1}{10}$ th of the total length. Interestingly both experiments show phase slips between the dominant and overtone contractions between the 70 min and 80 min mark indicating a corresponding adaption in phase difference.

a.5.5 Shells of revolution: Strain-displacement relations and energy considerations for an peristaltically contracting tube

We follow [122] to derive the relation between strain and displacement in a shell that is cylindrical in its reference state. This is equivalent to the "Approximation of Small Strains and Moderately Small Rotations" in [123]. Assuming a Hookean stress-strain relation, we then derive the elastic energy of this shell and we calculate the energy for harmonic deformation 


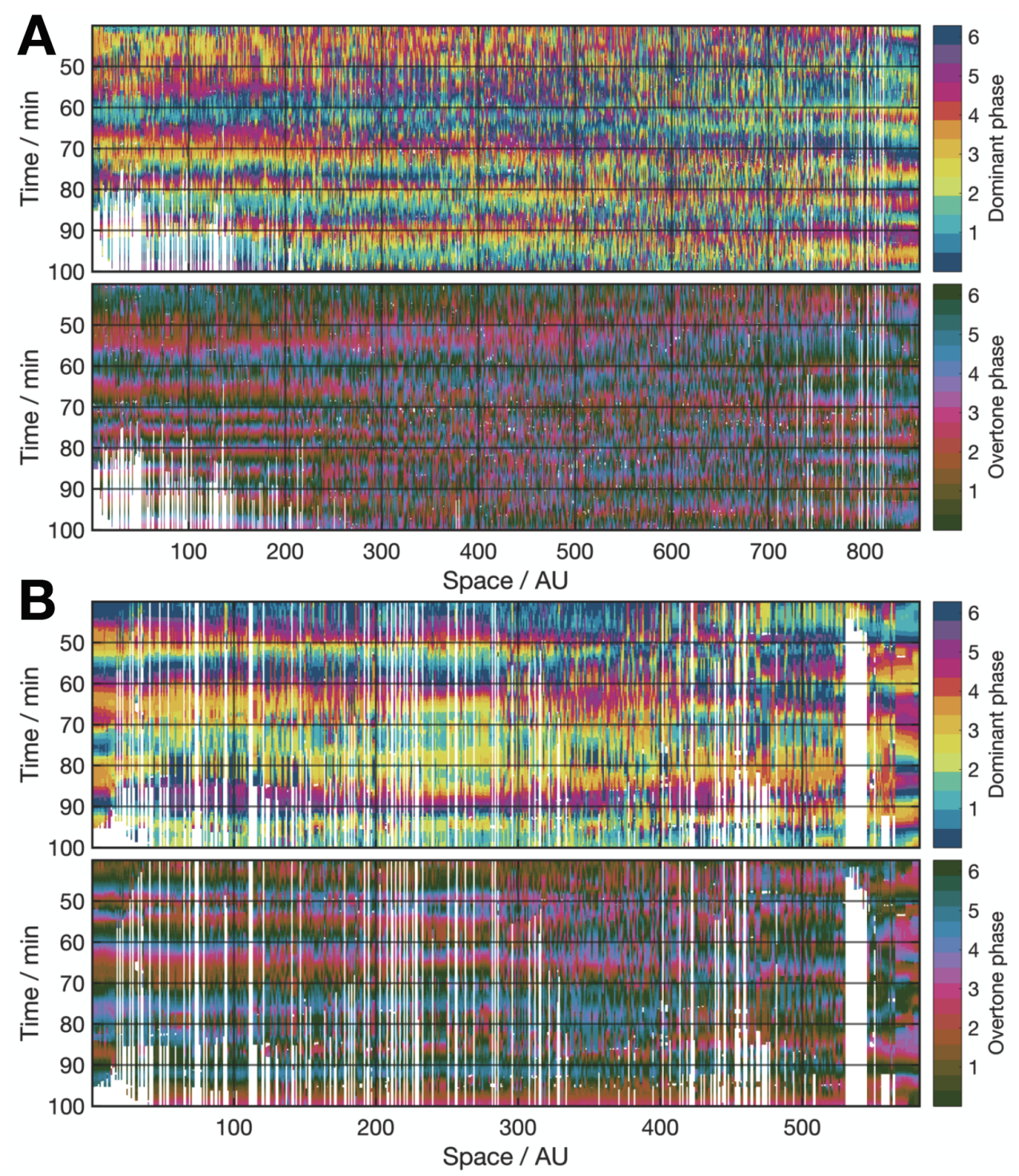

Figure A5: Contraction patterns in Physarum polycephalum span the system size even under blue light. Panel (A) and (B) show the dominant and overtone (second harmonic) phase for two exemplary data sets with a blue light stimulus at the 60 min mark. The phase is corrected for the contractions themselves, hence show drifting in case the overall respective frequency is adapting in the respective regions. 
with a second harmonic. Finally, we wish to derive a non-linear tractiondisplacement relation from a variation of the elastic energy with respect to the deformation, to be used in the elastic deformation model.

notation \& general case For thin axisymmetric shells in twist-free deformation, Audoly \& Pomeau [122] arrive at

$$
\begin{aligned}
& e_{s}=\rho^{\prime}(s) v_{r}^{\prime}(s)+z^{\prime}(s) v_{z}^{\prime}(s)+\frac{\varphi^{2}(s)}{2} \\
& e_{\theta}=\frac{v_{r}(s)}{\rho(s)}
\end{aligned}
$$

for the relation between membrane strains $e$ and displacements $v$. Here, $e_{s}$ is the strain in contour direction along the shell, $e_{\theta}$ is the strain in azimuthal direction (in the given approximation simply the hoop strain), $\rho(s)$ and $z(s)$ are, respectively, radius and axial coordinate of the shell as a function of the contour coordinate $s$ in reference configuration. $v_{r}(s)$ and $v_{z}(s)$ are the radial and axial displacement components, and $\varphi(s)$ is a measure for the leading order rotation of the surface relative to the reference configuration:

$$
\varphi(s)=-z^{\prime}(s) v_{r}^{\prime}(s)+\rho^{\prime}(s) v_{z}^{\prime}(s) .
$$

The bending strains are

$$
\begin{aligned}
& k_{s}(s)=\varphi^{\prime}(s) \\
& k_{\theta}(s)=\frac{v_{z}^{\prime}(s)}{\rho(s)},
\end{aligned}
$$

in contour- and azimuthal direction, respectively.

The energy density per unit surface area of the reference configuration for a Hookean shell material of thickness $h$ reads

$$
\begin{aligned}
& \phi_{s}=\frac{E h}{2\left(1-v^{2}\right)}\left(e_{s}^{2}+e_{\theta}^{2}+2 v e_{s} e_{\theta}\right), \\
& \phi_{b}=\frac{E h^{3}}{24\left(1-v^{2}\right)}\left(k_{s}^{2}+k_{\theta}^{2}+2 v k_{s} k_{\theta}\right),
\end{aligned}
$$

for stretch and bending, respectively, with $v$ the Poisson's ratio.

energy density for cylinders For a cylindrical shell of mid-plane radius $H_{0}, \rho(s)=H_{0}$ and $z^{\prime}(s)=1$, leading to 


$$
\begin{aligned}
& e_{s}=v_{z}^{\prime}(s)+\frac{v_{r}^{\prime 2}(s)}{2}, \\
& e_{\theta}=\frac{v_{r}(s)}{H_{0}}, \\
& k_{s}=-v_{r}^{\prime \prime}(s) \\
& k_{\theta}=\frac{v_{z}^{\prime}(s)}{H_{0}} .
\end{aligned}
$$

and energy densities become

$$
\begin{aligned}
& \phi_{s}=\frac{E h}{2\left(1-v^{2}\right)}\left(\left(v_{z}^{\prime}(s)+\frac{v_{r}^{\prime 2}(s)}{2}\right)^{2}+\left(\frac{v_{r}(s)}{H_{0}}\right)^{2}+2 v\left(v_{z}^{\prime}(s)+\frac{v_{r}^{\prime 2}(s)}{2}\right) \frac{v_{r}(s)}{H_{0}}\right), \\
& \phi_{b}=\frac{E h^{3}}{24\left(1-v^{2}\right)}\left(v_{r}^{\prime \prime 2}(s)+\left(\frac{v_{z}^{\prime}(s)}{H_{0}}\right)^{2}-2 v v_{r}^{\prime \prime}(s) \frac{v_{z}^{\prime}(s)}{H_{0}}\right) .
\end{aligned}
$$

scales \& approximations Here we assume a separation of scales

$$
v_{z} \ll h \sim v_{r} \ll H_{0} \ll \lambda,
$$

meaning that the wavelength $\lambda$ is much larger than the undeformed tube radius $H_{0}$, and that the dominant deformation $v_{r}$ occurs in radial direction, is much smaller than the undeformed tube radius $H_{0}$, but probably comparable to the wall thickness $h$ of the tube. For quantitative references for these approximations see Sec. A.5.2.

Scaling the contour $s$ with $\lambda$, and $v_{r}$ and $v_{z}$ with $\epsilon H_{0}(\epsilon \ll 1)$, the energy density, expanded and sorted by their order of magnitude, is

$$
\begin{aligned}
& \phi_{s}=\frac{E h \epsilon^{2}}{2\left(1-v^{2}\right)}\left(\quad v_{r}^{2}(s)+2 v \frac{H_{0}}{\lambda} v_{z}^{\prime}(s) v_{r}(s)+\left(\frac{H_{0}}{\lambda}\right)^{2} v_{z}^{\prime 2}(s)\right. \\
& \left.+v \epsilon\left(\frac{H_{0}}{\lambda}\right)^{2} v_{r}^{\prime 2}(s) v_{r}(s)+\epsilon\left(\frac{H_{0}}{\lambda}\right)^{3} v_{z}^{\prime}(s) v_{r}^{\prime 2}(s)+\epsilon^{2}\left(\frac{H_{0}}{\lambda}\right)^{4} \frac{v_{r}^{\prime 4}(s)}{4}\right), \\
& \phi_{b}=\frac{E h \epsilon^{2}}{24\left(1-v^{2}\right)} \frac{h^{2}}{\lambda^{2}}\left(v_{z}^{\prime 2}(s)-2 v \frac{H_{0}}{\lambda} v_{r}^{\prime \prime}(s) v_{z}^{\prime}(s)+\left(\frac{H_{0}}{\lambda}\right)^{2} v_{r}^{\prime \prime 2}(s)\right) .
\end{aligned}
$$

Furthermore, we did not record any axial movement of the tubes in any of the experiments and consequently assume that the axial displacement $v_{z}$ is negligible in comparison to the radial displacement $v_{r}, v_{z} \ll \frac{\epsilon H_{0}}{\lambda} v_{r}$, due 
to the fixation of the specimen to the substrate surface. Then the elastic energy density up to the first non-linear term reads, in physical units:

$$
\phi_{s}=\frac{E h}{2\left(1-v^{2}\right)}\left(\frac{v_{r}^{2}(s)}{H_{0}{ }^{2}}+v \frac{v_{r}^{\prime 2}(s)}{H_{0}} v_{r}(s)\right)
$$

\section{a.5.6 Perturbative approach to peristalsis in elastic tubes}

We consider a simple deformable tube of equilibrium radius $H_{0}$ that is subjected to the fluid pressure within it, and model the peristaltic wave of wavelength $\lambda$ and velocity $V$ as an imposed active normal traction. Initially we follow the approach of Takagi and Balmforth [111], treating the fluid flow in a long-wave approximation $\left(\lambda \gg H_{0}\right)$ :

$$
u_{x}=\frac{1}{4 \mu} \frac{d p}{d x}\left(r^{2}-H^{2}\right)-V
$$

where $x$ is the axial coordinate, $r$ is the radial coordinate, $u_{x}$ is the axial velocity of the fluid, measured in the frame co-moving with the peristaltic wave of velocity $V, p$ is the pressure in the fluid, $H$ is the tube radius, and $\mu$ is the fluid viscosity. Integrating Eq. (46) gives the flux in the co-moving frame,

$$
q=-\pi\left(\frac{H^{4}}{8 \mu} \frac{d p}{d x}+V H^{2}\right)
$$

and mass conservation implies

$$
\frac{d}{d x}\left\{\frac{H^{4}}{8 \mu} \frac{d p}{d x}+V H^{2}\right\}=0
$$

An integral of Eq. (48) gives again the flux and the constant of integration can be found by imposing an integral condition on $H$ [111]. The tube radius $H$ is coupled to the pressure and the imposed forcing by some constitutive equation which, in a general form, is

$$
F[H]=p-f
$$

where $f$ is the imposed force and $F$ is a functional of $H$ that could contain non-local relations in space and time. We restrict ourselves to the simplest nonlinear viscoleastic tube, modeled by

$$
K\left(H-H_{0}\right)+\eta \partial_{t} H+\beta\left(H-H_{0}\right)^{n}=p-f,
$$


where $K$ and $\eta$ represent elastic and damping coefficients of the tube, $\beta$ quantifies the nonlinearity of the tube behavior and $n>1$ the order of the nonlinear term.

We do not intend here to accurately model an existing rheology or tube model, but rather to capture the fundamental consequences of some dissipative component in the wall response and the simplest possible nonlinearity. The elastic constant $K$ follows from a variation of Eq. (45) as $K=$ $\frac{E h}{\left(1-v^{2}\right) H_{0}^{2}}$. Note, however, that the corresponding (geometrical) non-linearity has a negligible magnitude with physiological conditions and a different functional form than the non-linearity in Eq. (50). The latter should rather be interpreted as emerging from an active process or a material property of the actin cortex.

Using Eq. (50) in Eq. (48), we switch to the dimensionless co-moving coordinate $\bar{\xi}=2 \pi(x / \lambda-t / T)$, where $T=\lambda / V$, the pumping period. We scale $H$ with $H_{0}, f$ with $f_{0}$, the characteristic forcing amplitude, and $F$ with $K H_{0}$, the scale of the elastic part in the constitutive model. From now on we continue with the dimensionless variables:

$$
\frac{d}{d \bar{\zeta}}\left\{H^{4}\left(\left(1+\alpha(H-1)^{n-1}\right) \frac{d H}{d \bar{\zeta}}-\gamma \frac{d^{2} H}{d \bar{\zeta}^{2}}+\rho \frac{d f}{d \bar{\zeta}}\right)+\kappa H^{2}\right\}=0,
$$

with

$$
\alpha=\frac{n \beta H_{0}^{n-1}}{K}, \quad \gamma=\frac{2 \pi \eta V}{K \lambda}, \quad \rho=\frac{f_{0}}{K H_{0}}, \quad \kappa=\frac{4 V \mu \lambda}{\pi H_{0}^{3} K}
$$

where $\alpha$ is a measure for the nonlinearity in the tube elasticity, $\gamma$ is the Deborah number which relates the intrinsic material time scale $\eta / K$ to the pumping frequency $V / \lambda$, and $\kappa$ in an elasto-hydrodynamic coupling coefficient.

We now treat Eq. (51) perturbatively, assuming small perturbations around the force-free radius $H_{0}$. The deflection scale relative to the force-free radius $H_{0}$ is given by $\epsilon$, which we will consider small in the following. Thus we expand

$$
H \sim 1+\epsilon \delta_{1}+\epsilon^{2} \delta_{2}+\mathcal{O}\left(\epsilon^{3}\right),
$$

where $\delta_{1}$ and $\delta_{2}$ will be associated to the fundamental and the overtone of the deflection, respectively. Inserting Eq. (53) into Eq. (51) yields 


$$
\begin{gathered}
\frac{d}{d \bar{\zeta}}\left\{( 1 + \epsilon \delta _ { 1 } + \epsilon ^ { 2 } \delta _ { 2 } ) ^ { 4 } \epsilon \left(\left(1+\alpha\left(\epsilon \delta_{1}+\epsilon^{2} \delta_{2}\right)^{n-1}\right)\left(\frac{d \delta_{1}}{d \bar{\zeta}}+\epsilon \frac{d \delta_{2}}{d \bar{\zeta}}\right)-\ldots\right.\right. \\
\left.\left.-\gamma\left(\frac{d^{2} \delta_{1}}{d \bar{\zeta}^{2}}+\epsilon \frac{d^{2} \delta_{2}}{d \bar{\zeta}^{2}}\right)+\frac{d f}{d \bar{\zeta}}\right)+\kappa\left(1+\epsilon \delta_{1}+\epsilon^{2} \delta_{2}\right)^{2}\right\}=0
\end{gathered}
$$

This equation can now be solved separately for each order of $\epsilon$ [112]:

$$
\begin{array}{r}
\mathcal{O}(\epsilon): \\
\frac{d}{d \bar{\zeta}}\left\{\frac{d \delta_{1}}{d \bar{\xi}}-\gamma \frac{d^{2} \delta_{1}}{d \bar{\xi}^{2}}+2 \kappa \delta_{1}+\frac{d f}{d \bar{\zeta}}\right\}=0 \\
\mathcal{O}\left(\epsilon^{2}\right): \frac{d}{d \bar{\xi}}\left\{4 \delta_{1}\left(\frac{d \delta_{1}}{d \bar{\xi}}+\frac{d f}{d \bar{\xi}}-\gamma \frac{d^{2} \delta_{1}}{d \bar{\zeta}^{2}}\right)+\alpha \delta_{1} \frac{d \delta_{1}}{d \bar{\xi}}+\frac{d \delta_{2}}{d \bar{\zeta}}-\gamma \frac{d^{2} \delta_{2}}{d \bar{\zeta}^{2}}+\kappa\left(\delta_{1}^{2}+2 \delta_{2}\right)\right\}=0
\end{array}
$$

where we have assumed $n=2$, the simplest possible non-linearity. We now consider a forcing in the form of a traveling harmonic wave $f=-\cos \bar{\xi}$ and find

$$
\delta_{1}=\frac{1}{\sqrt{1+(2 \kappa+\gamma)^{2}}} \cos (\bar{\zeta}+\arctan (2 \kappa+\gamma))+c_{1},
$$

as periodic solutions for Eq. (55). Using Eq. (57) in Eq. (56) and solving for $\delta_{2}$, we find the same structure for the overtone. For the linear viscoelastic case $(\alpha \rightarrow 0)$, the solution is

$$
\delta_{2}=\frac{7 \kappa}{4\left(1+(2 \kappa+\gamma)^{2}\right) \sqrt{1+(\kappa+2 \gamma)^{2}}} \cos \left(2 \bar{\xi}+2 \arctan (2 \kappa+\gamma)+\arctan (\kappa+2 \gamma)-\frac{\pi}{2}\right)+c_{2}
$$

with twice the frequency and a phase shift of

$$
\Delta \vartheta_{\alpha \rightarrow 0}=\frac{\pi}{2}-\arctan (\kappa+2 \gamma) \text {. }
$$

Note that $\Delta \vartheta$ is defined as in the experiments where the phase difference between $\delta_{2}$ and $\delta_{1}$ is measured for positive cosine overtones, i.e. from the maximum positive (outward) deflection in the spatial coordinate of the overtone. Hence $\Delta \vartheta=2 \arg \delta_{1}-\arg \delta_{2}$. Since $\gamma>0$ and $\kappa>0$ is of $\mathcal{O}(1)$, a linear viscoelastic foundation can not lead to the observed phase relation $\Delta \vartheta>\pi / 2$ in the forced case. Still, it agrees well with the observed values in the nonforces case. 
The nonlinear solution is most instructively analysed in terms of a small non-linearity, and we therefore expand the full solution around $\alpha=0$ :

$$
\delta_{2}=\frac{\left(7 \kappa+\frac{\alpha^{2}}{14 \kappa}\right) \cos \left(2 \bar{\zeta}+2 \arctan (2 \kappa+\gamma)+\arctan (\kappa+2 \gamma)-\frac{\alpha}{7 \kappa}-\frac{\pi}{2}\right)}{4\left(1+(2 \kappa+\gamma)^{2}\right) \sqrt{1+(\kappa+2 \gamma)^{2}}}+c_{2},
$$

omitting any terms on the order of $\mathcal{O}\left((\alpha / \kappa)^{3}\right)$ and beyond. The phase difference in the same approximation is

$$
\begin{aligned}
\Delta \vartheta_{\alpha \ll \kappa} & =\pi / 2-\arctan (\kappa+2 \gamma)+\frac{\alpha}{7 \kappa} \\
& =\pi / 2-\arctan \left(\kappa+4 \pi \frac{\eta V}{K \lambda}\right)+\frac{2 \beta H_{0}}{7 K \kappa} .
\end{aligned}
$$

The expansion actually only contains the ratio $\alpha / \kappa$, meaning that it requires $\alpha \ll \kappa$. For $\kappa \ll 1$, even a small non-linearity could have an $\mathcal{O}(1)$ effect on the phase relation. In case of strain stiffening $(\alpha>0)$ the phase difference can therefore move along the positive flank of the unit circle as observed in experiments.

\section{A.6 List of experiments for 'Spatial mapping reveals multi-step pattern of wound healing in Physarum polycephalum'}

This section provides a complete list of acquired data sets.

Each experiment is a figure with two sub-figures displaying: a) the raw images of the plasmodium at the beginning of the experiment (left), immediately after the cut is performed (middle), and at the end of experiment (right). The cut site is marked with a red cross and the section of the network chosen for kymograph analysis is marked with a red line. The scale bar always represents $1 \mathrm{~mm}$ and is shown in the latest image. b) shows the results of kymograph analysis. Here, the black bar, if applicable, denotes stalling.

The figures are complemented with a detailed descriptions of the morphological dynamics based on visual inspection of the bright field data. Both quantification and visual inspection together allow to identify if stalling does happen or not as discussed for each data set.

The experiments are sorted in a descending order by the magnitude of stalling. The organisms in experiments from Fig. A6 to Fig. A12 show most pronounced stalling, followed by a weaker display of stalling from Fig. A13 to Fig. A20. The organisms in experiments from Fig. A21 to Fig. A26 do not show a clear stalling behaviour. 

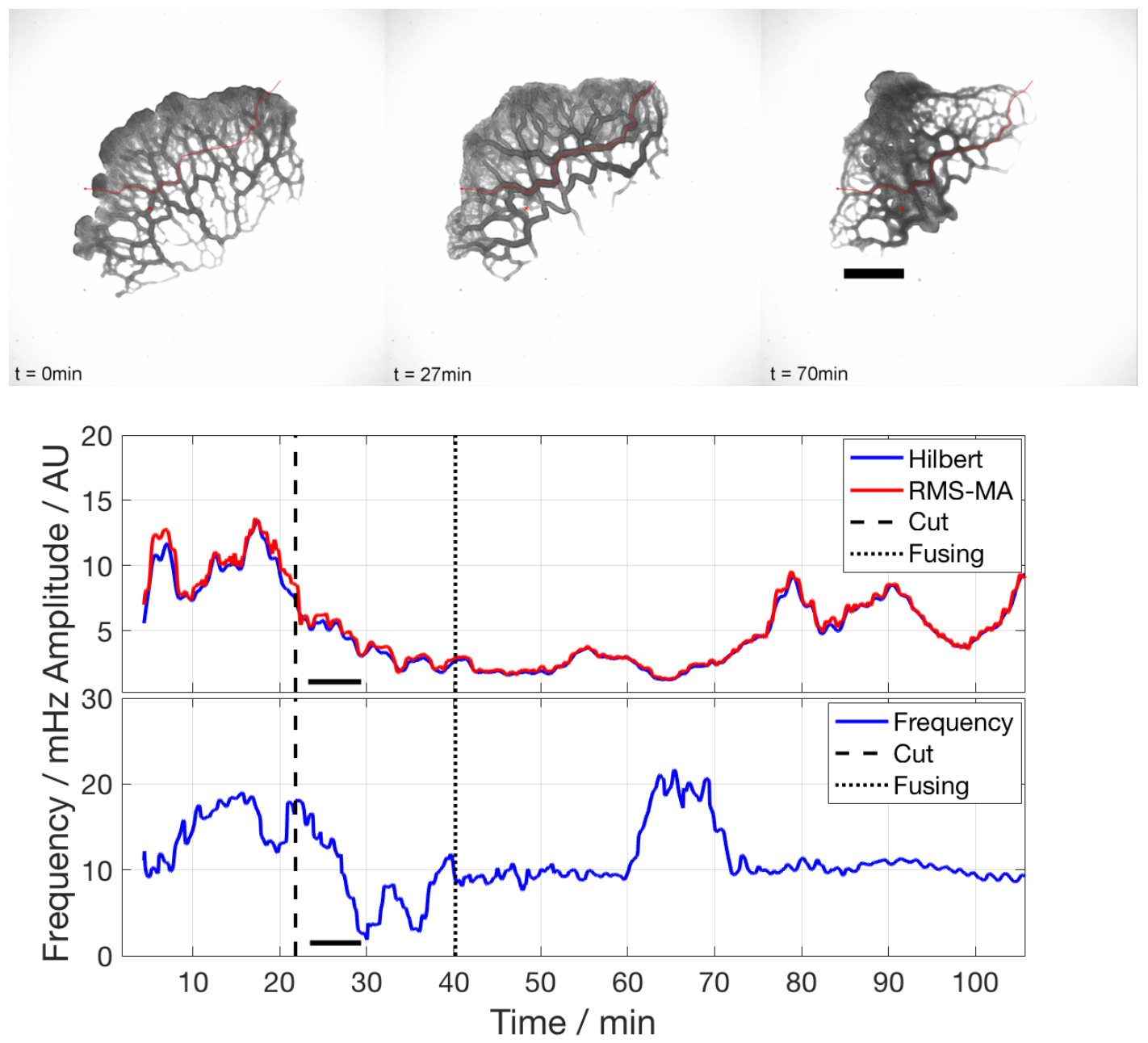

Figure A6: A small cut is performed across two of the thicker tube in this branched network, separating it into a large and small sub-network. A fan is first built around the cut site, then disintegrates as the flow is reestablished. After an initial delay, the organism experiences stalling. 

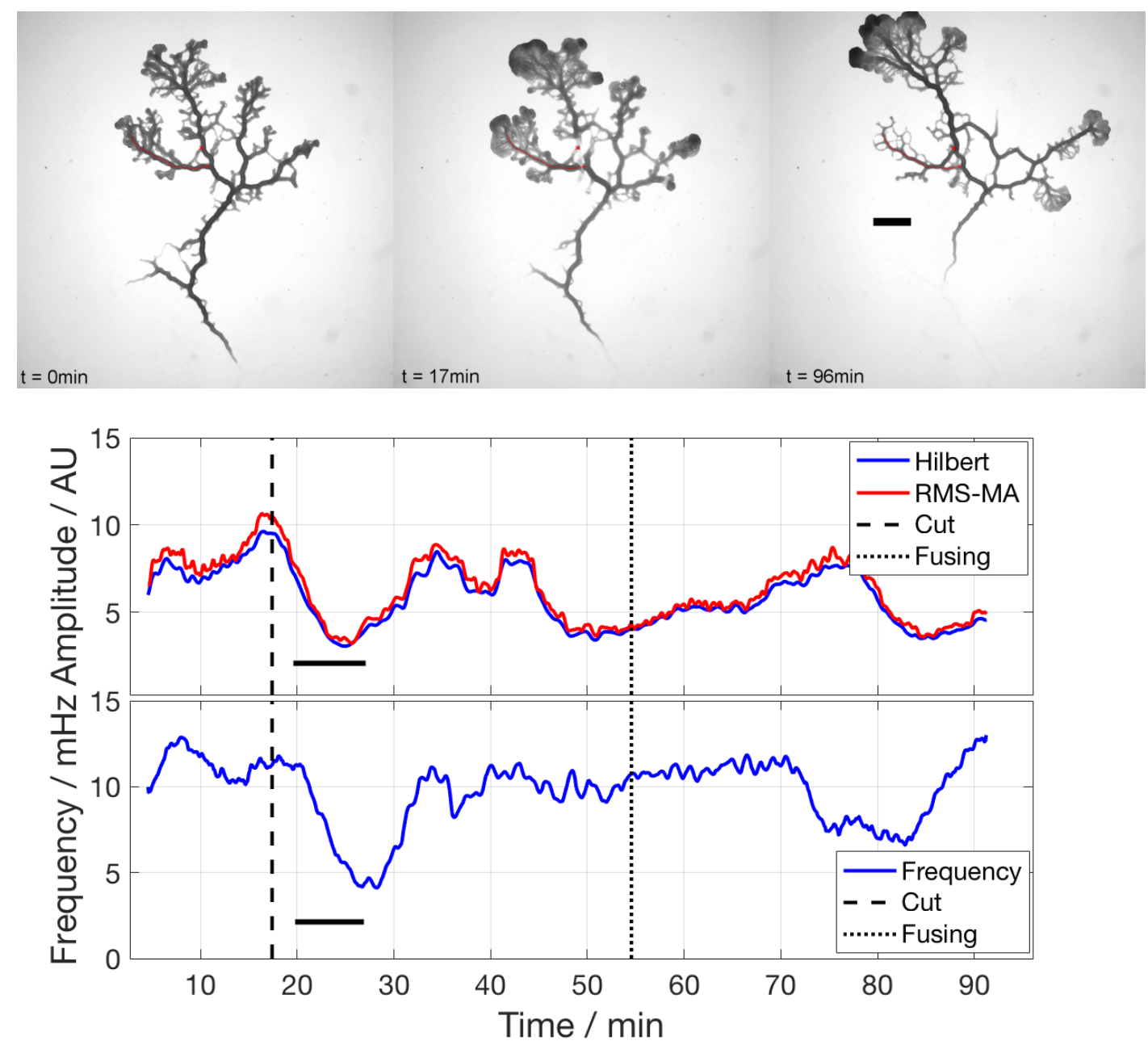

Figure A7: This data set is prominently described within the main text. We refer to the results section (3) for detailed description. 

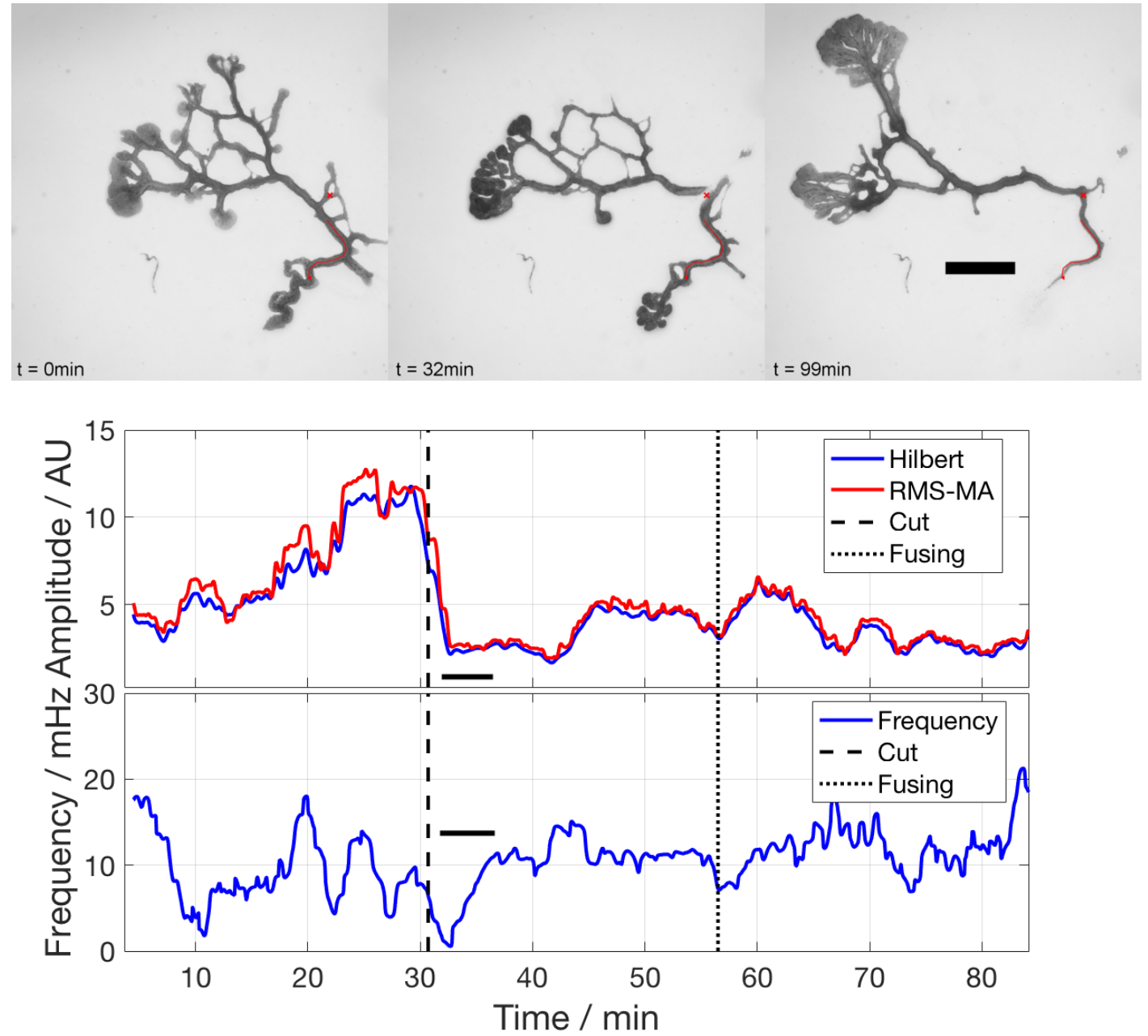

Figure A8: This small plasmodium with few tubes was cut across the thickest tube connecting the two fan regions. The cut removed a part of the tube and hence completely separated the two regions. Stalling is immediate after the cut, seen as a drop in amplitude and frequency. As the cut ends rejoin, a fan is built around the cut side. This experiment was fully analysed with quantitative analysis (see Fig.S5). 

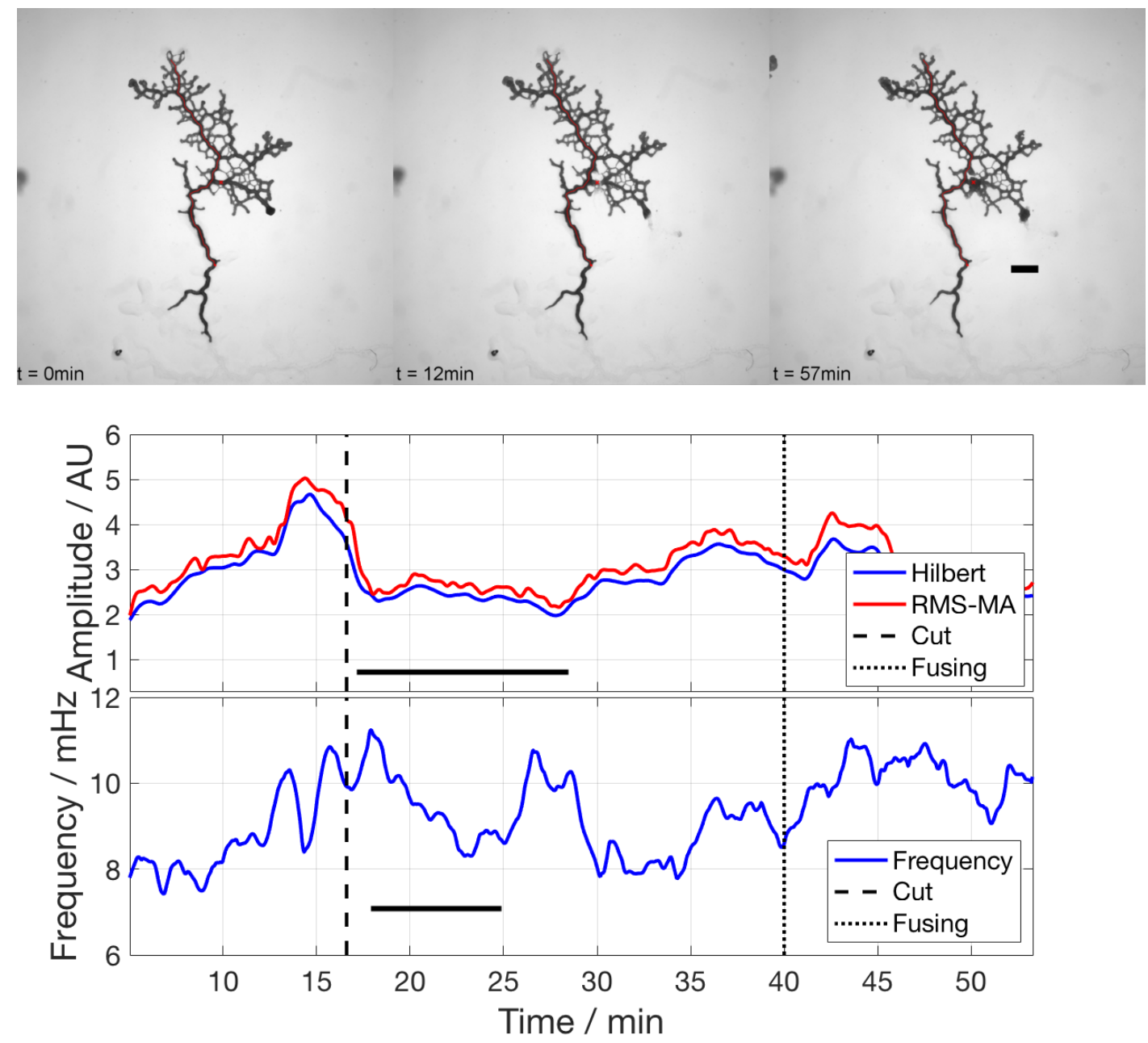

Figure A9: In this larger network, a thicker side tube was cut. The cut slightly separated the ends of the tube which then rejoin while a large fan is built around the cut site. The organism experiences stalling, visible as a decrease in frequency and amplitude of the oscillations. The fan around the cut site maintains its size until the end of the experiment. 

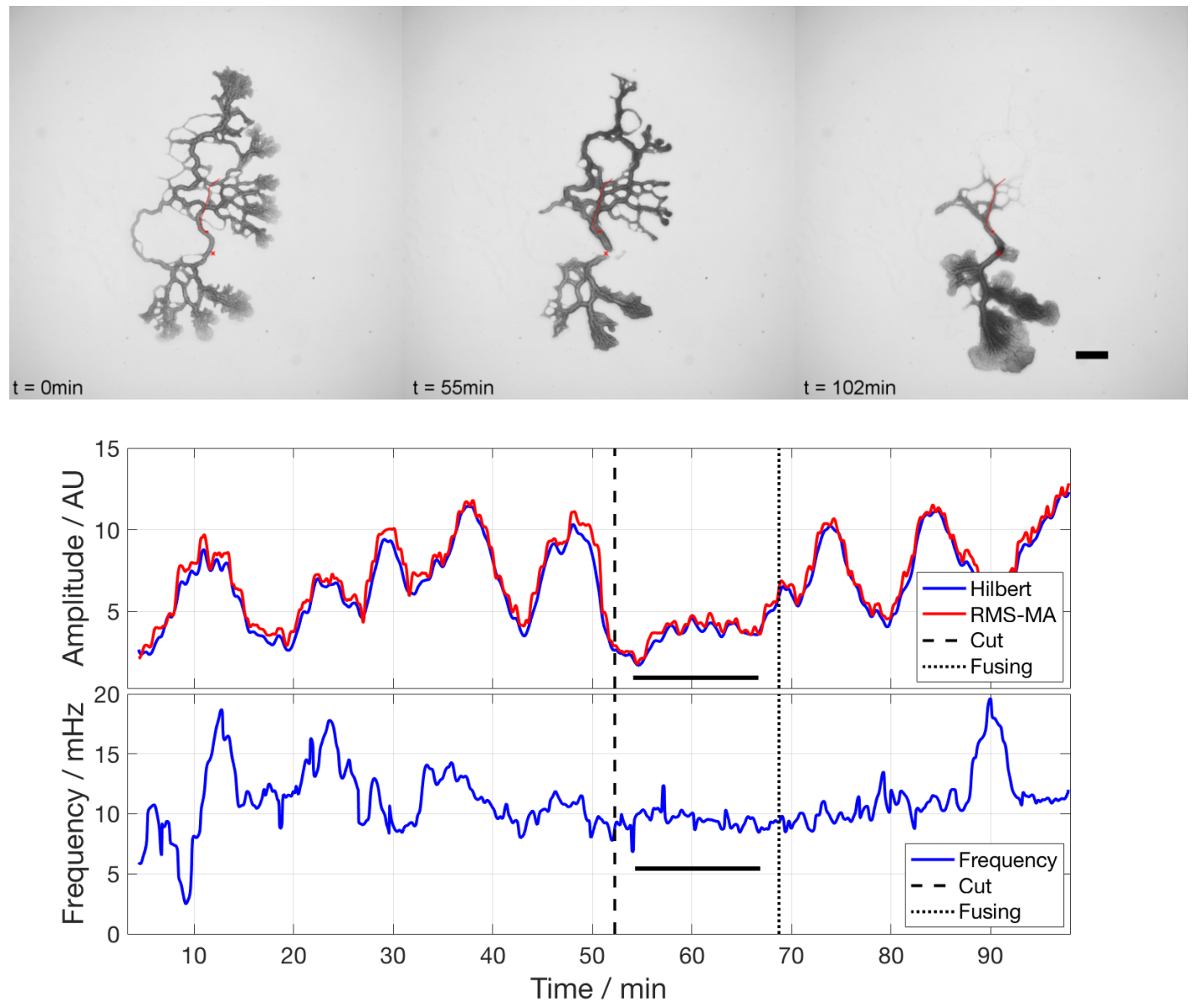

Figure A10: A cut was performed across a thick tube connecting two distant regions of the organism of comparable size, chosen such that the organism has no possibility of re-routing the flow through a close-by neighbouring tube. However, the cut barely separated the tube ends and they rejoin quickly. A fan is growing around the cut site. The stalling in both sub-networks appears in connection with very low variation both in amplitude and frequency of the oscillations compared to the pre-cut and post-rejoining phase. 

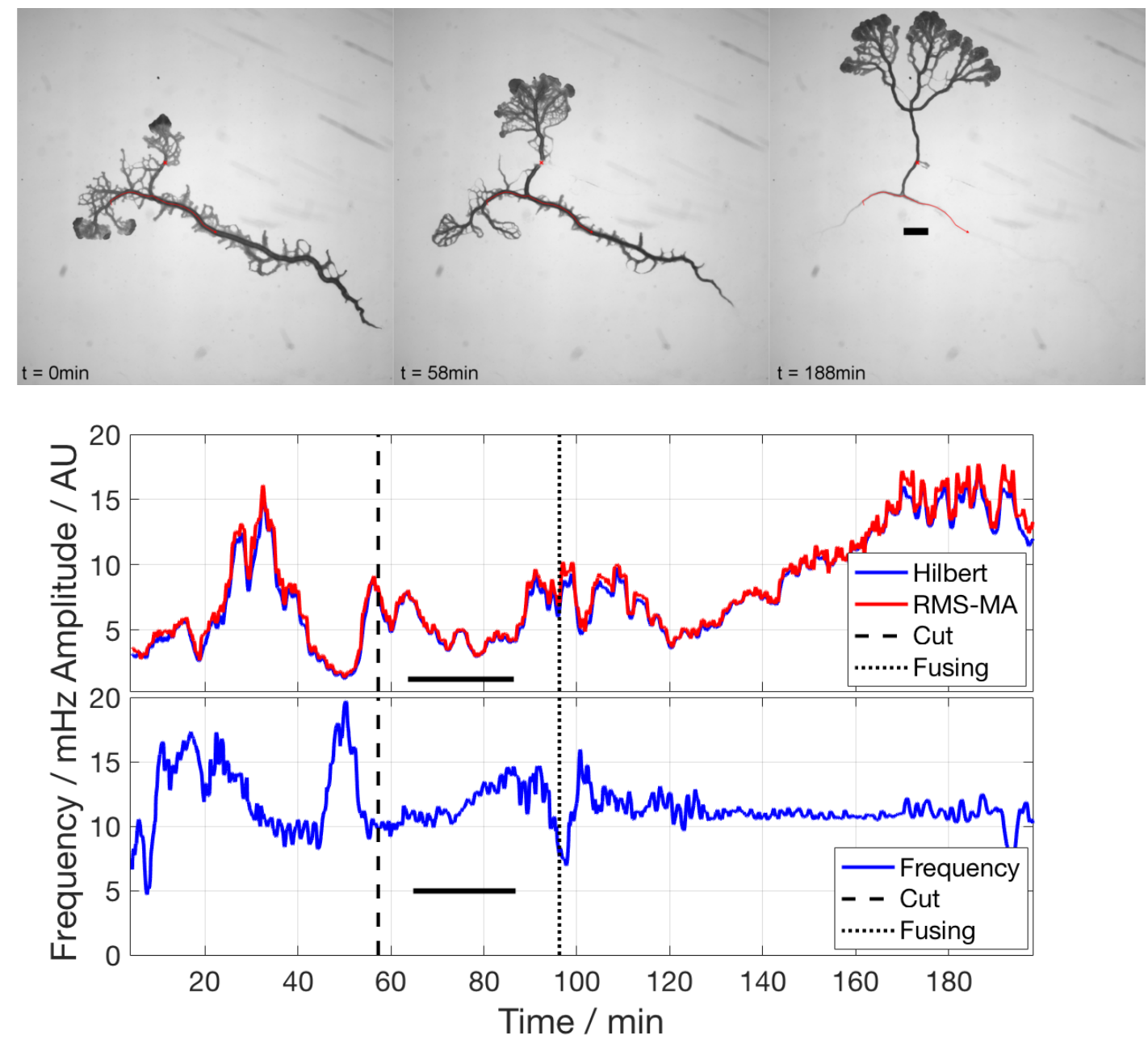

Figure A11: A cut is performed across a thick single tube connecting a large fan region with the rest of the organism. The amplitude and frequency of the oscillations stall first, followed by a gradual increase as the network rejoins the cut parts. Once the flow is re-established, the plasmodium prunes one side of the body and moves as a whole in the direction of the large fan. 

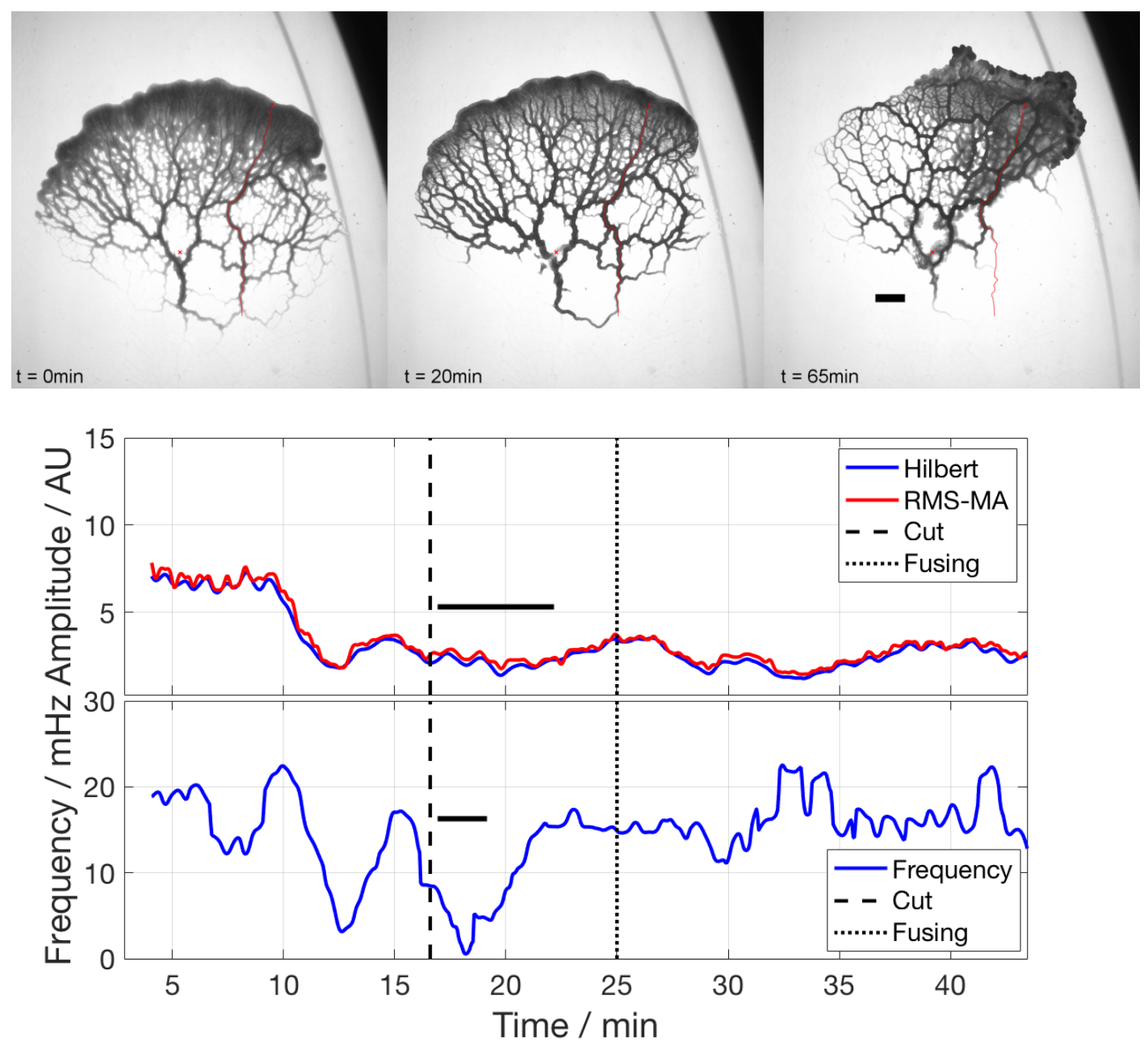

Figure A12: The morphology of this small network changes rapidly over the course of the experiment. A cut is performed across two neighbouring wide tubes at the base of the network. The tube segments fuse back completely as fans grow around it. Stalling is very prominent in this experiment and shows as a large drop in the frequency of the oscillations. 

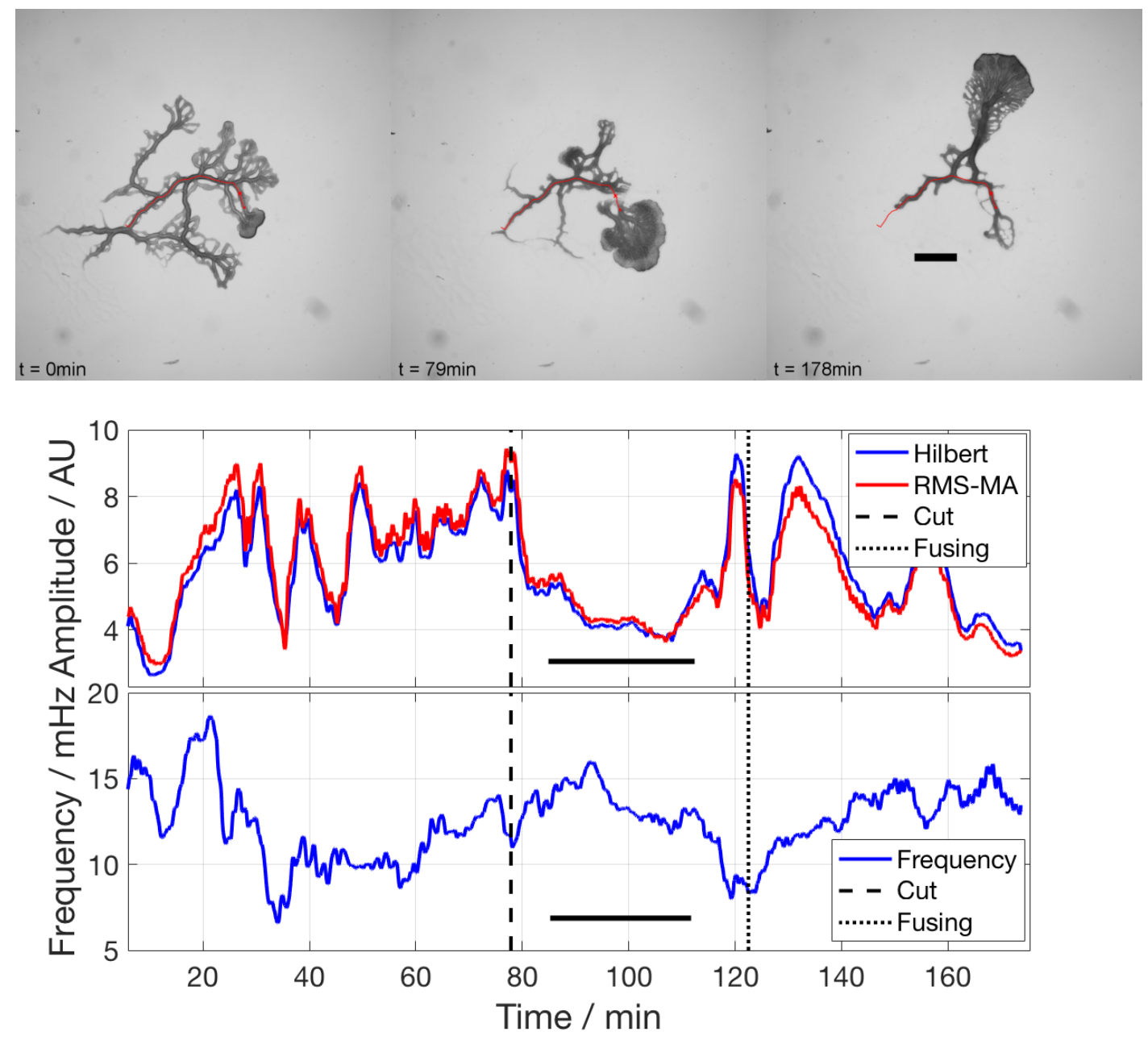

Figure A13: A cut is applied across the base of the only large fan in the network, resulting in damage of two thick tubes. The large fan, now separated, stalls and then builds body mass at the cut site. After rejoining, the organism as a whole builds a large fan and moves as a whole. 

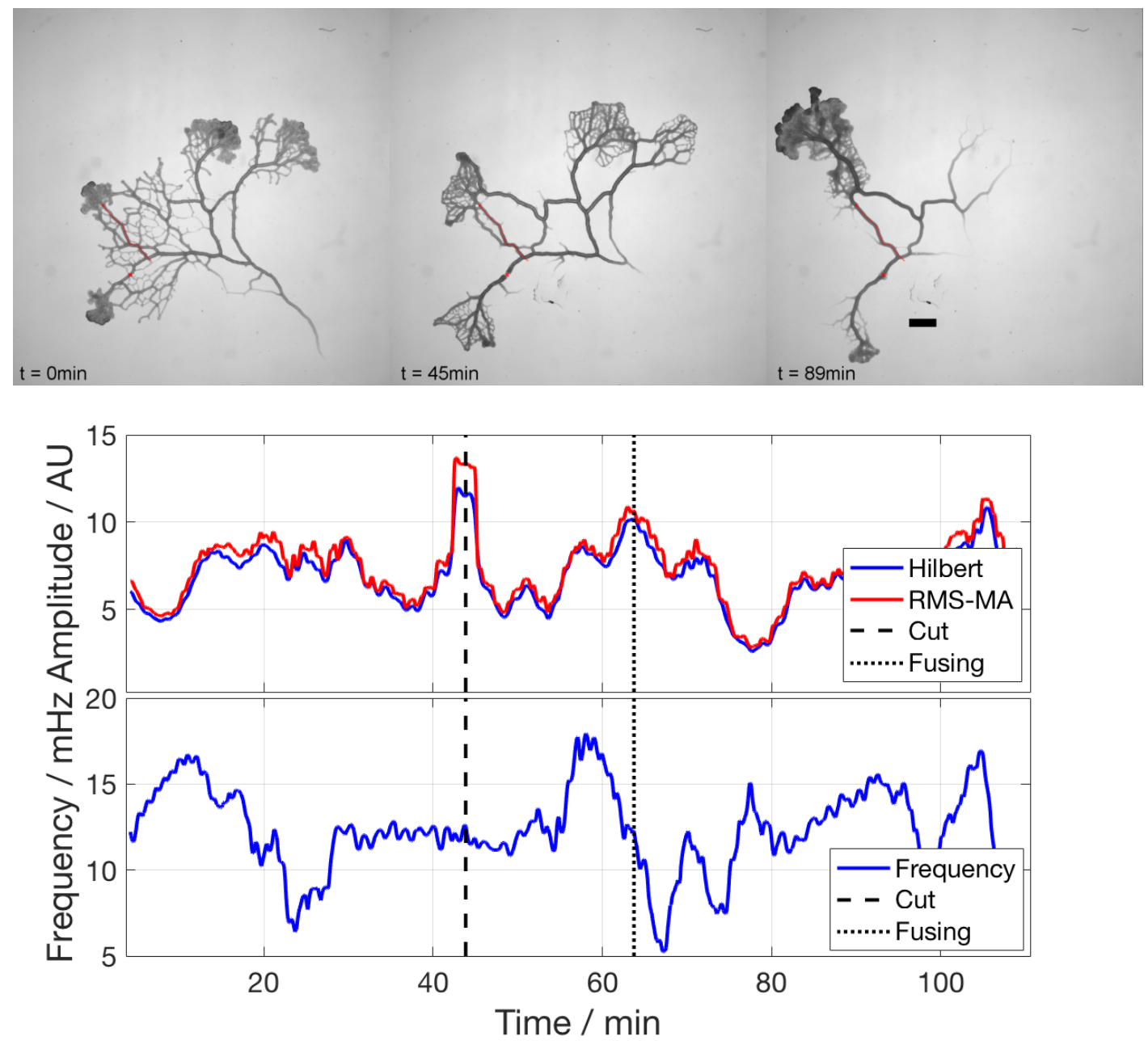

Figure A14: Here, four fan regions are initially growing and one of these is cut off at the only connection. The cut site is repaired fairly quickly with minimal fan growth. The amplitude and frequency hardly drop after the cut, yet fan growth in the periphery stalls and only resumes after flow is reinstated. After fusion the smaller sub-network is declining and fans are grown in the big sub-network. 

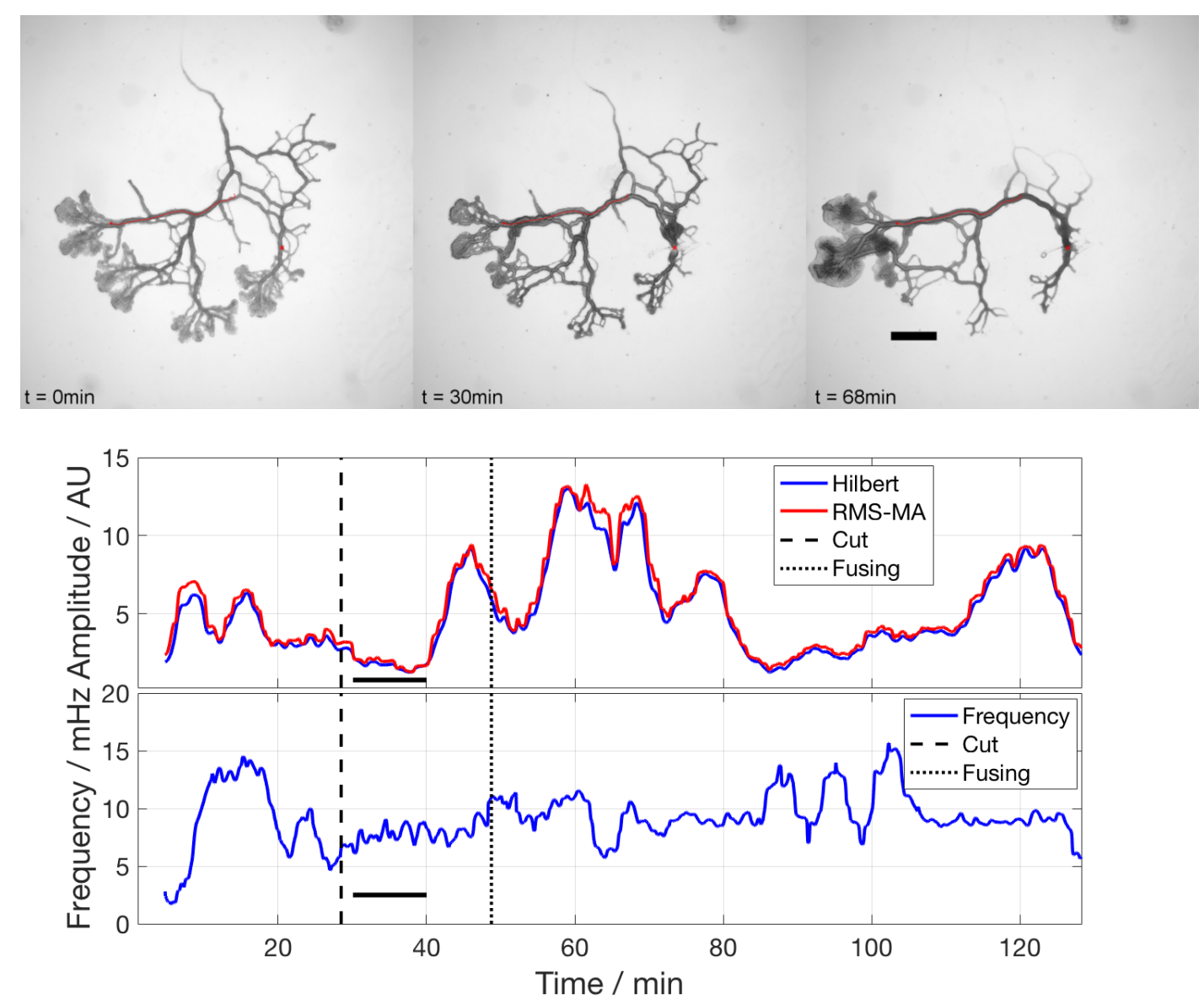

Figure A15: A cut is performed across one of the main tubes, distal from the region where most of the body mass is concentrated. The kymograph along the thickest tube shows stalling as a drop in the amplitude. A small fan grows at the site of the cut from both sub-networks. As the tubes refuse and the flow is established, the organism builds big fans and moves out of the imaging window. 

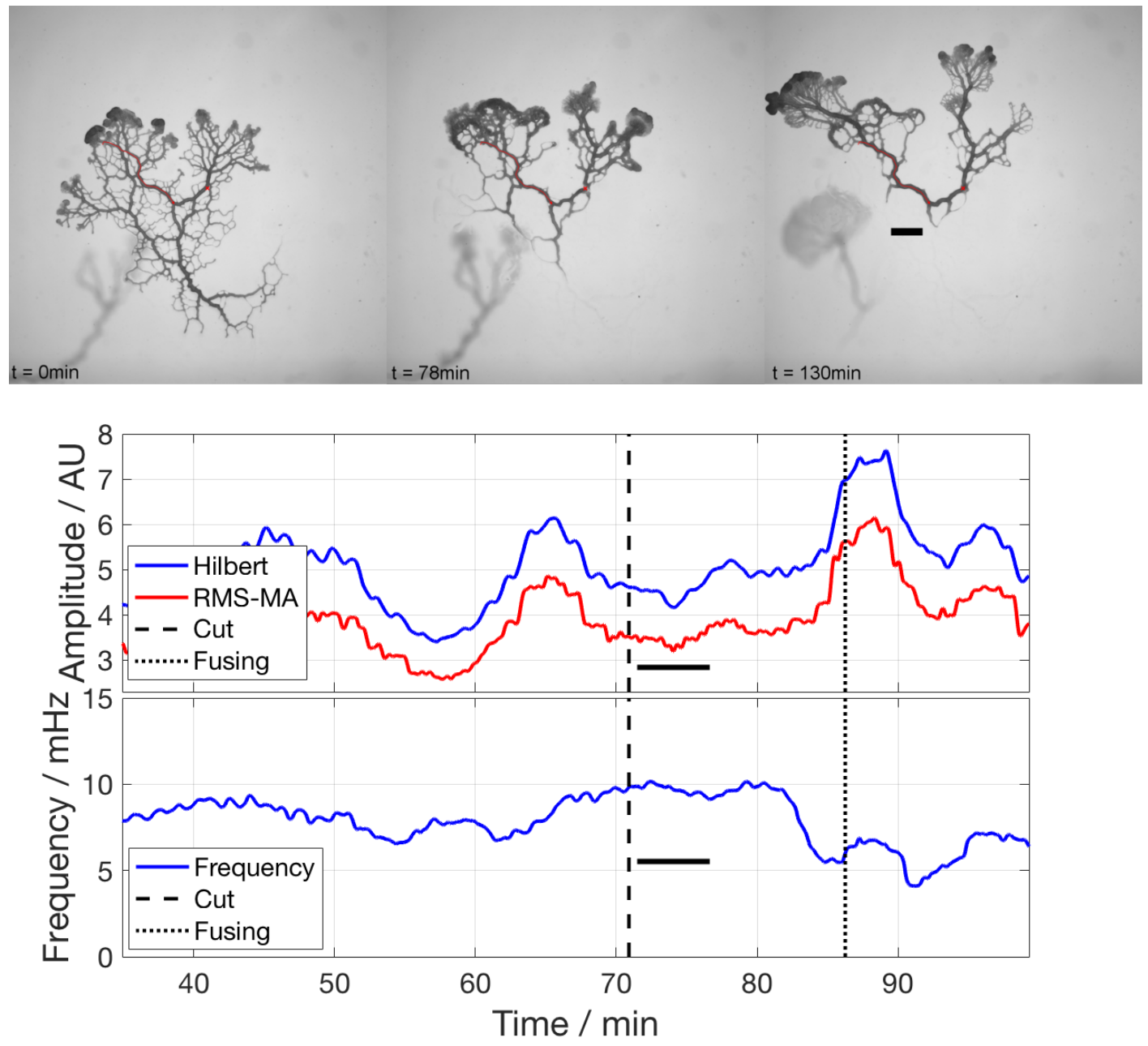

Figure A16: Initially the organism rapidly changes its morphology, likely as a response to light. A cut was performed across the biggest tube connecting the two fan regions, a site with no possibility of re-routing the flow through neighbouring tubes. The organism experiences slight stalling and quickly rejoins the cut tube ends. A small fan is built around the cut site. 

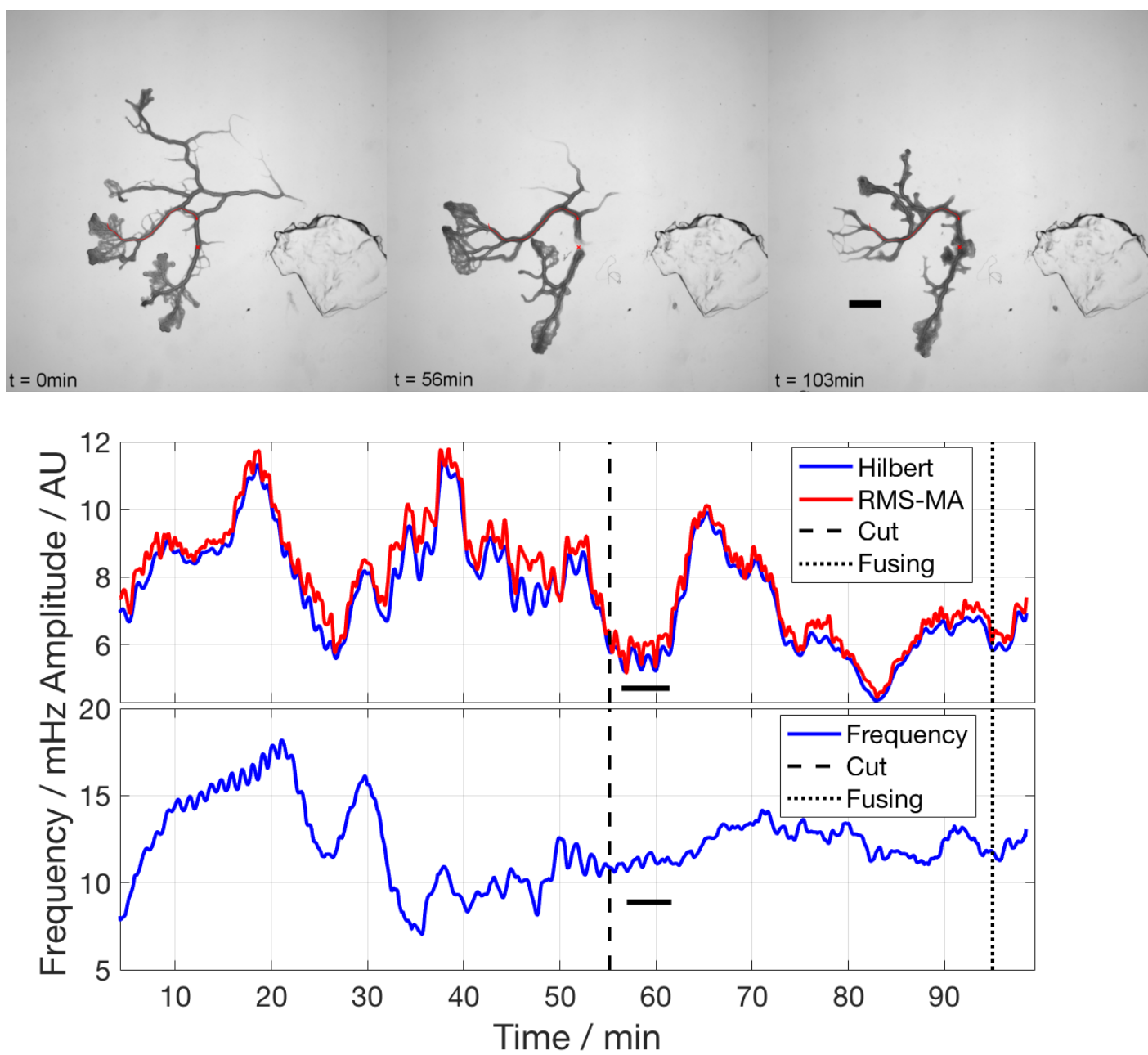

Figure A17: The organism experiences a putative light shock in the beginning indicated by the change in morphology. The cut is applied across a thick tube connecting two regions of comparable size and shape, separating them completely. The two sub-networks take a relatively long time to rejoin, meanwhile amassing new thick fans out of existing veins. Stalling is visible as a drop in amplitude. The general behaviour of this network suggests that the effects of the initial light shock persist throughout the whole experiment. 

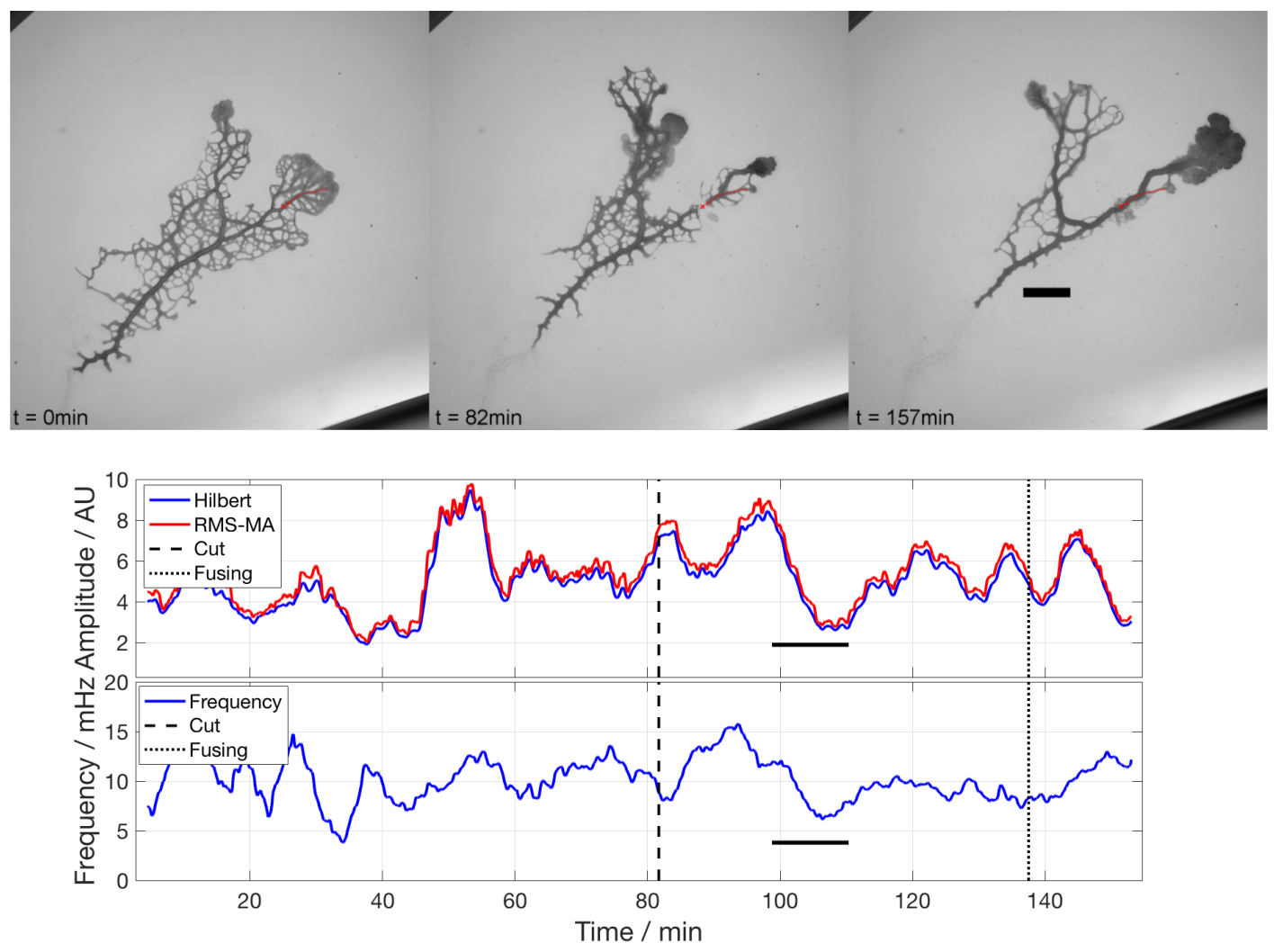

Figure A18: The cut is applied at the base of a smaller fan, followed by movement of the body mass in the fan towards the cut site. The fan in the larger sub-network retracts and the body mass moves towards the cut site. After the cut site is repaired and the sub-networks rejoin, the organism moves as a whole. The stalling occurs in both networks after the cut with a delay. 

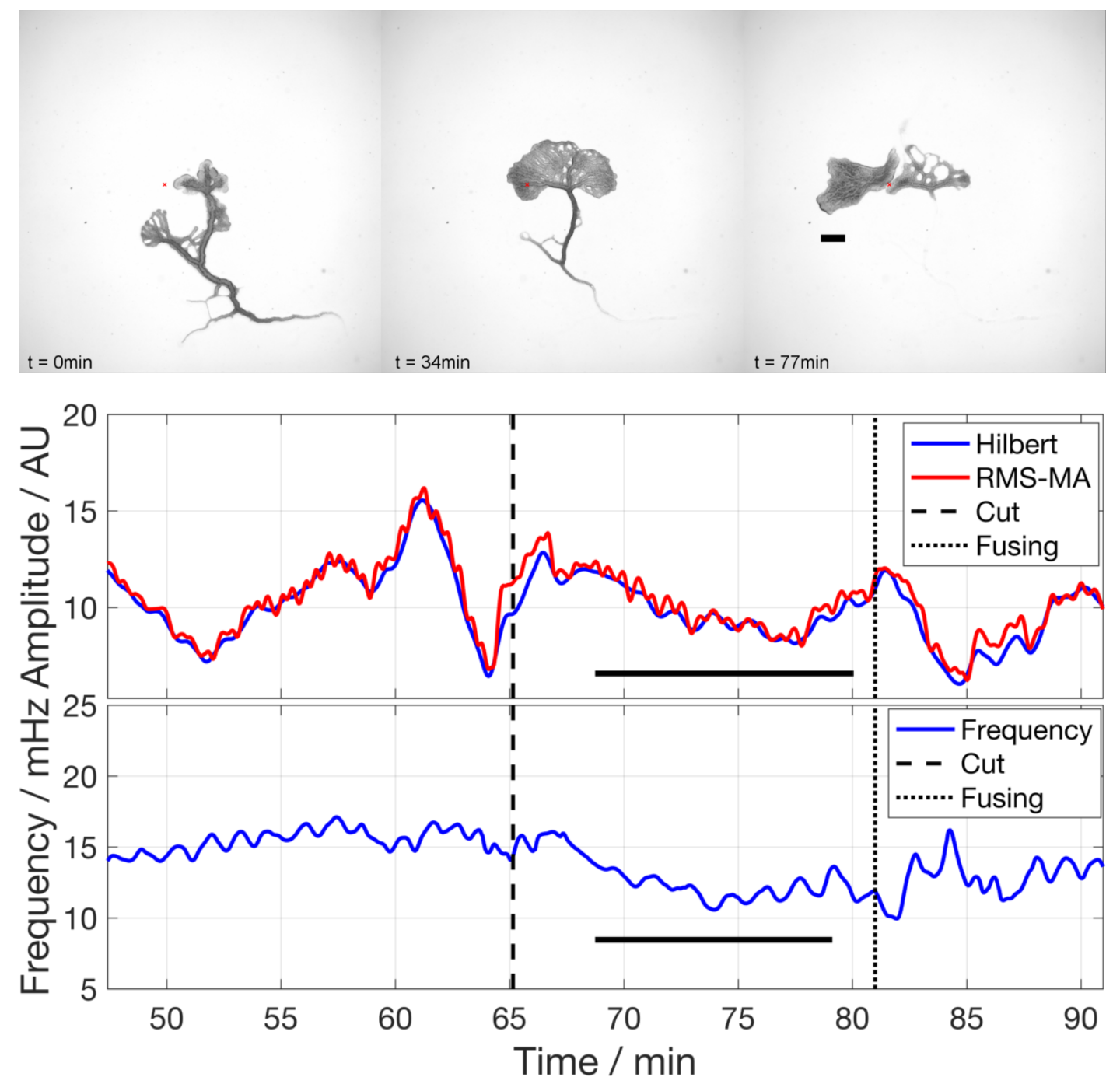

Figure A19: This plasmodium behaves rather like a very motile foraging fan than an extended network. There are hardly any hard distinctions between tube and fan in the main part of the plasmodium. The cut completely severs the specimen into two parts and stalling is evident, but it quickly returns to foraging behaviour. 

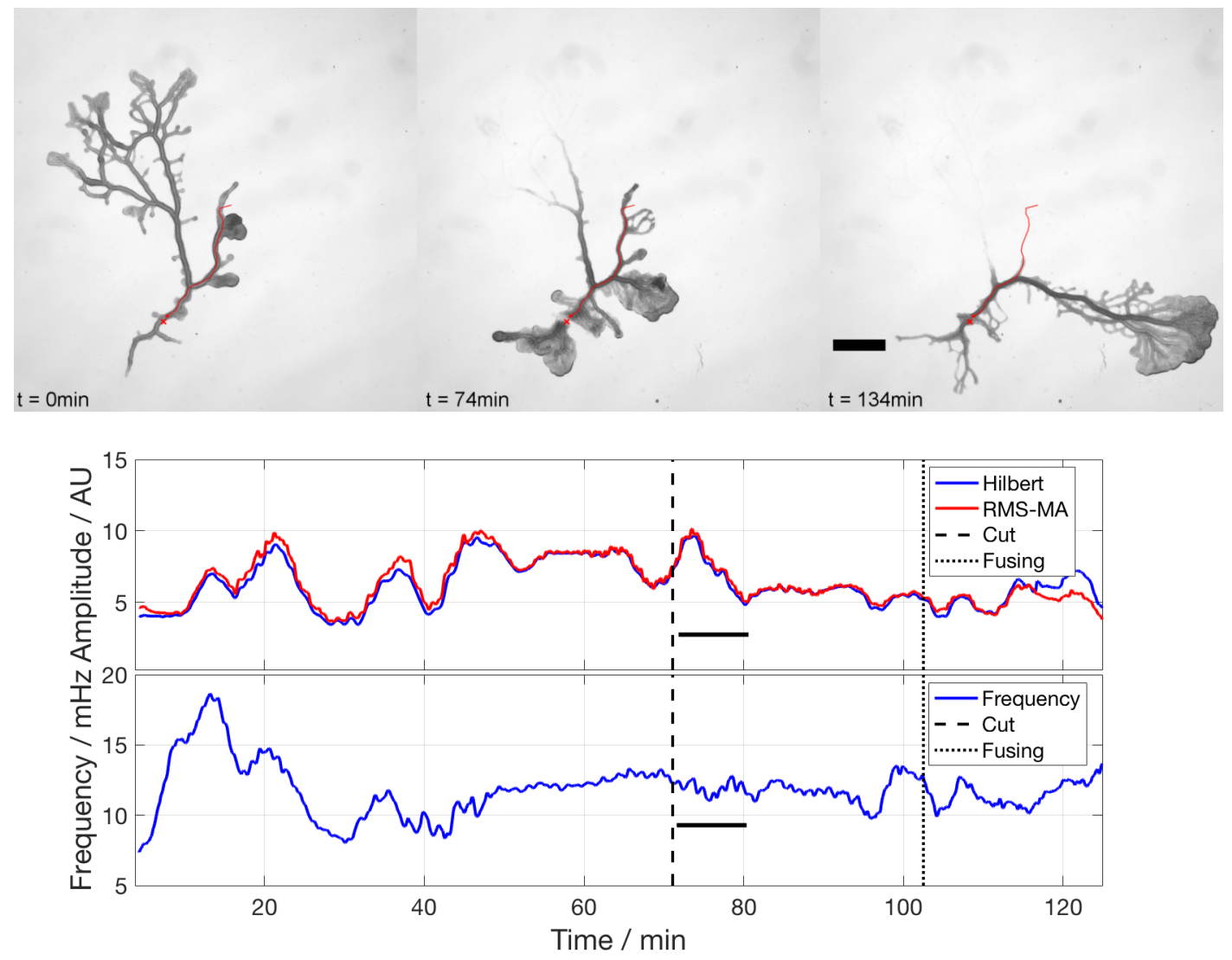

Figure A20: The network in this experiment initially shows a fast morphology change, possibly from illumination, and fan growth out of the sides of the tubes. A cut is performed along the base of a fan. The network experiences hardly any stalling, with a slight decrease in amplitude and frequency of the oscillations. The cut parts rejoin, the organism builds a large fan and moves in its direction. Is it possible that the initial effects persist during the whole experiment. 

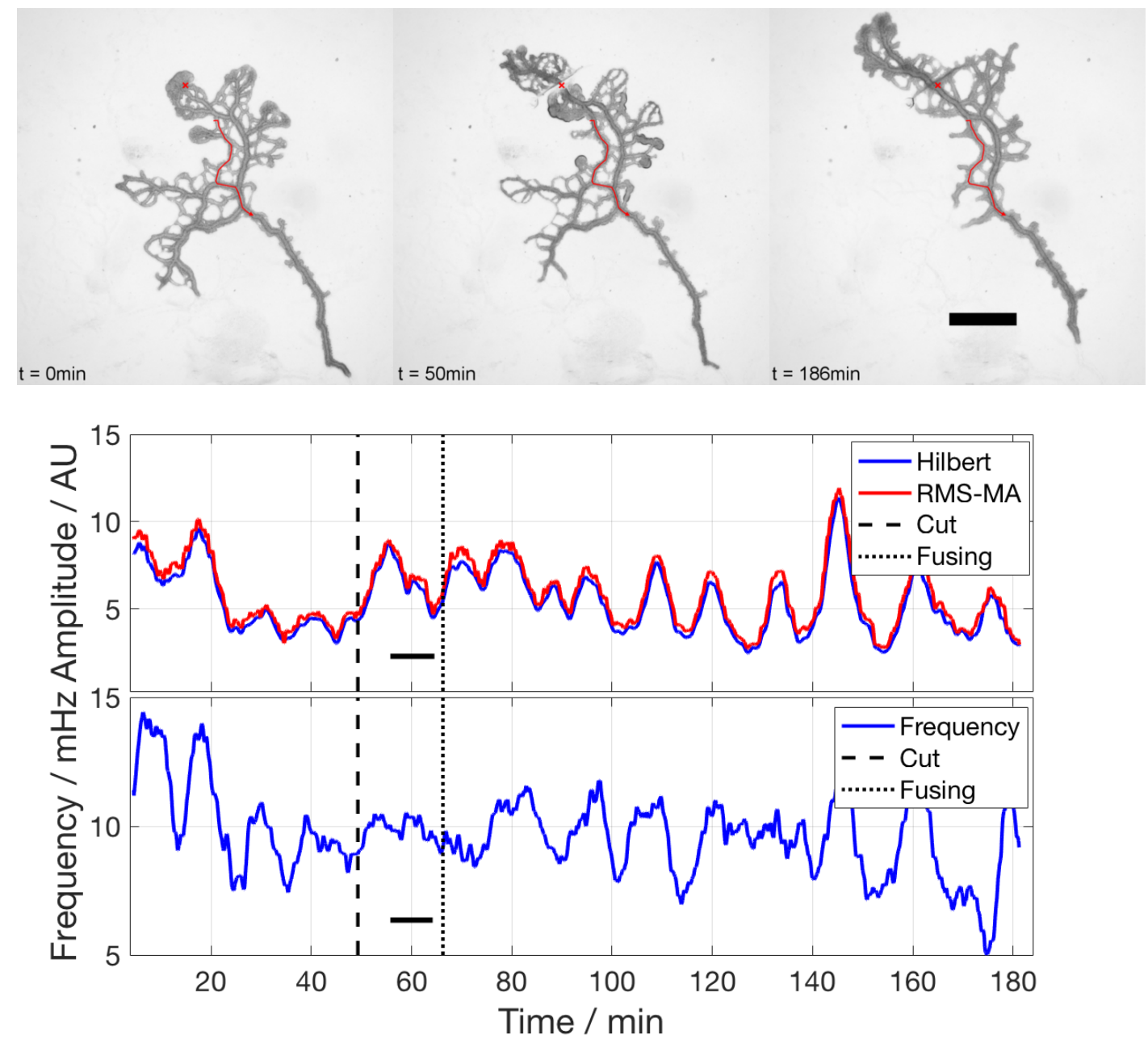

Figure A21: The plasmodium experiences a putative initial light shock which is indicated by a quick change of morphology, resulting in a network with a single fan. A cut was performed across the tube in the fan. The cut parts rejoin quickly. A thick fan is first grown at the cut site, then absorbed into the fan at the top of the image. No pronounced stalling is visible. 

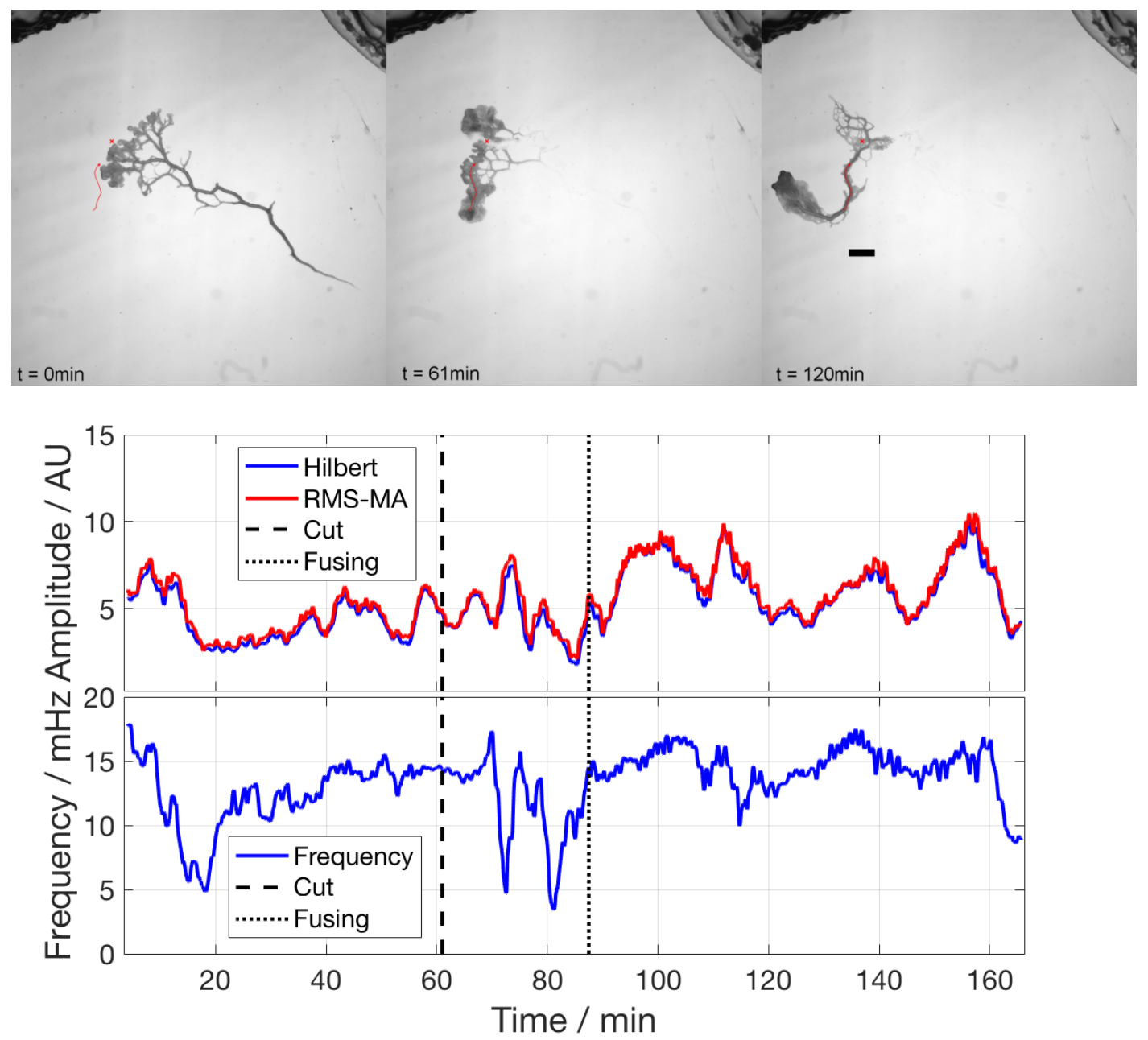

Figure A22: The plasmodium has high motility in this experiment. A cut is performed across a tube connecting two fan regions. After the cut, the material from the fan closer to the cut is moved towards the cut site and the cut parts rejoin. There is no obvious stalling. 

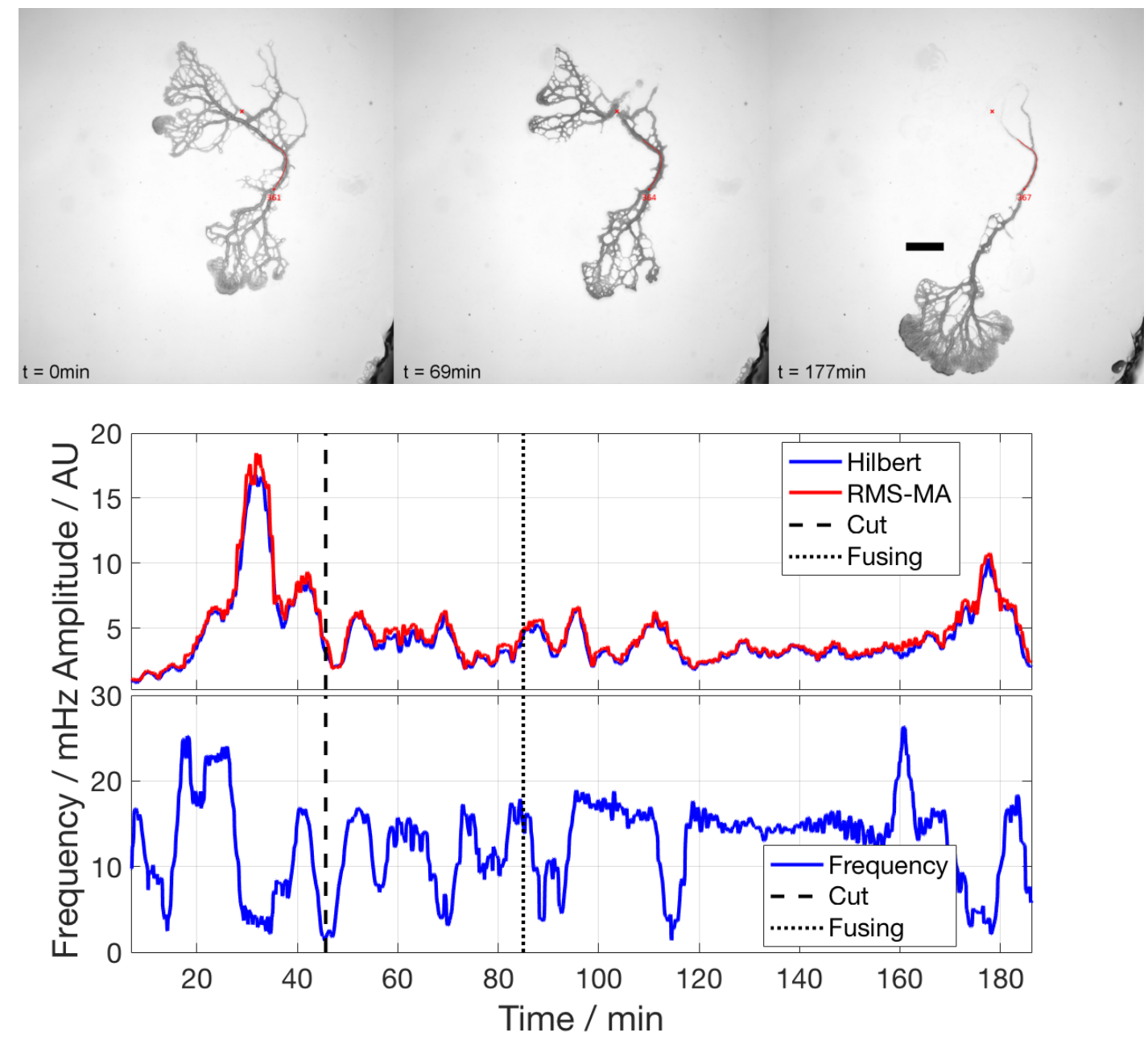

Figure A23: A cut is made across the thickest tube connecting two fan regions at the opposing ends of the organism. The organism builds a large, diffuse fan at the cut site and rejoins the cut parts. The network then moves as a whole. The variations in the frequency and amplitude of the osculations make the identification of stalling challenging. 

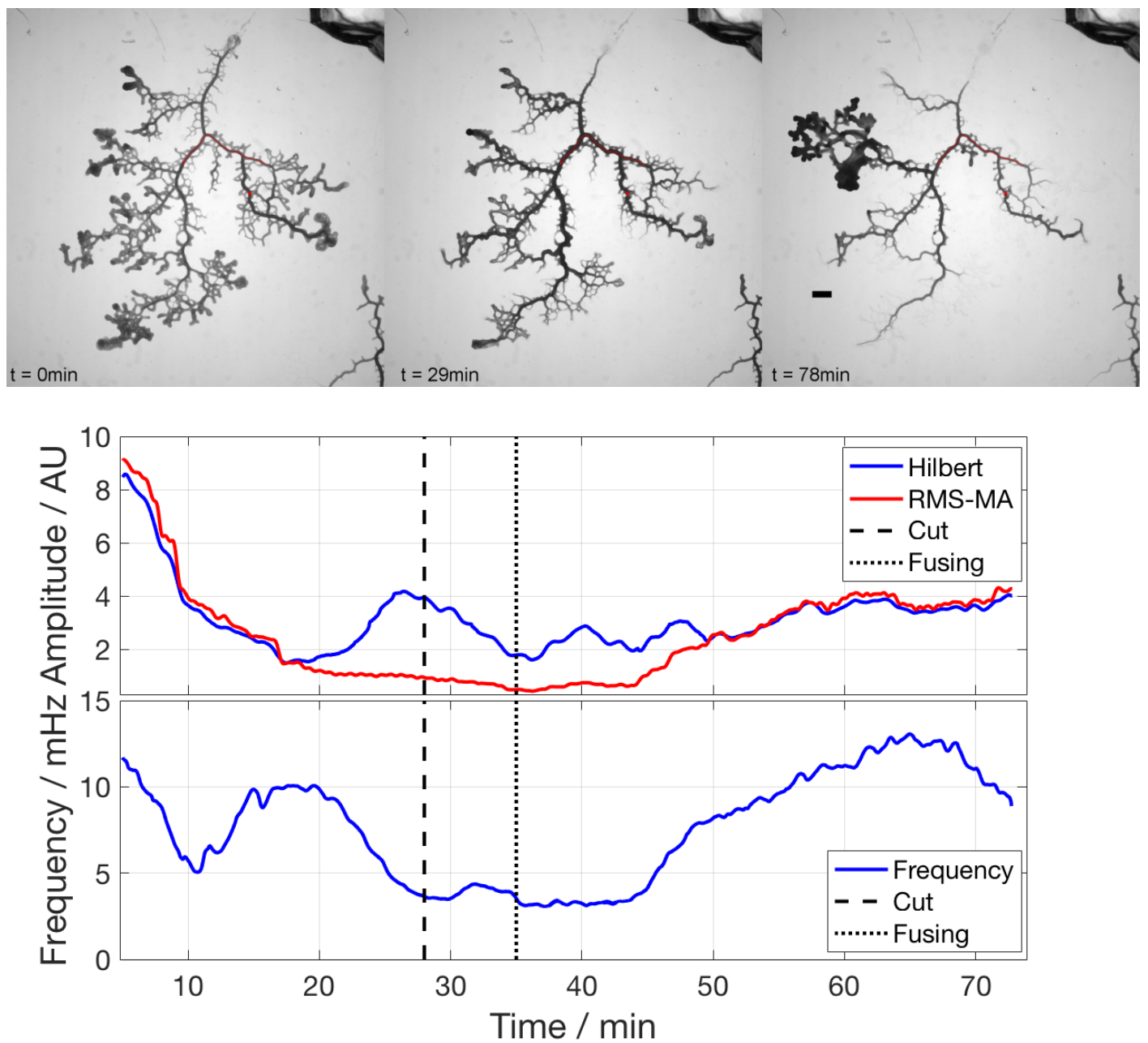

Figure A24: In this large branched network, a cut is applied across on of the thick veins, damaging it slightly. The cut parts re-join quickly and no stalling is observed. 

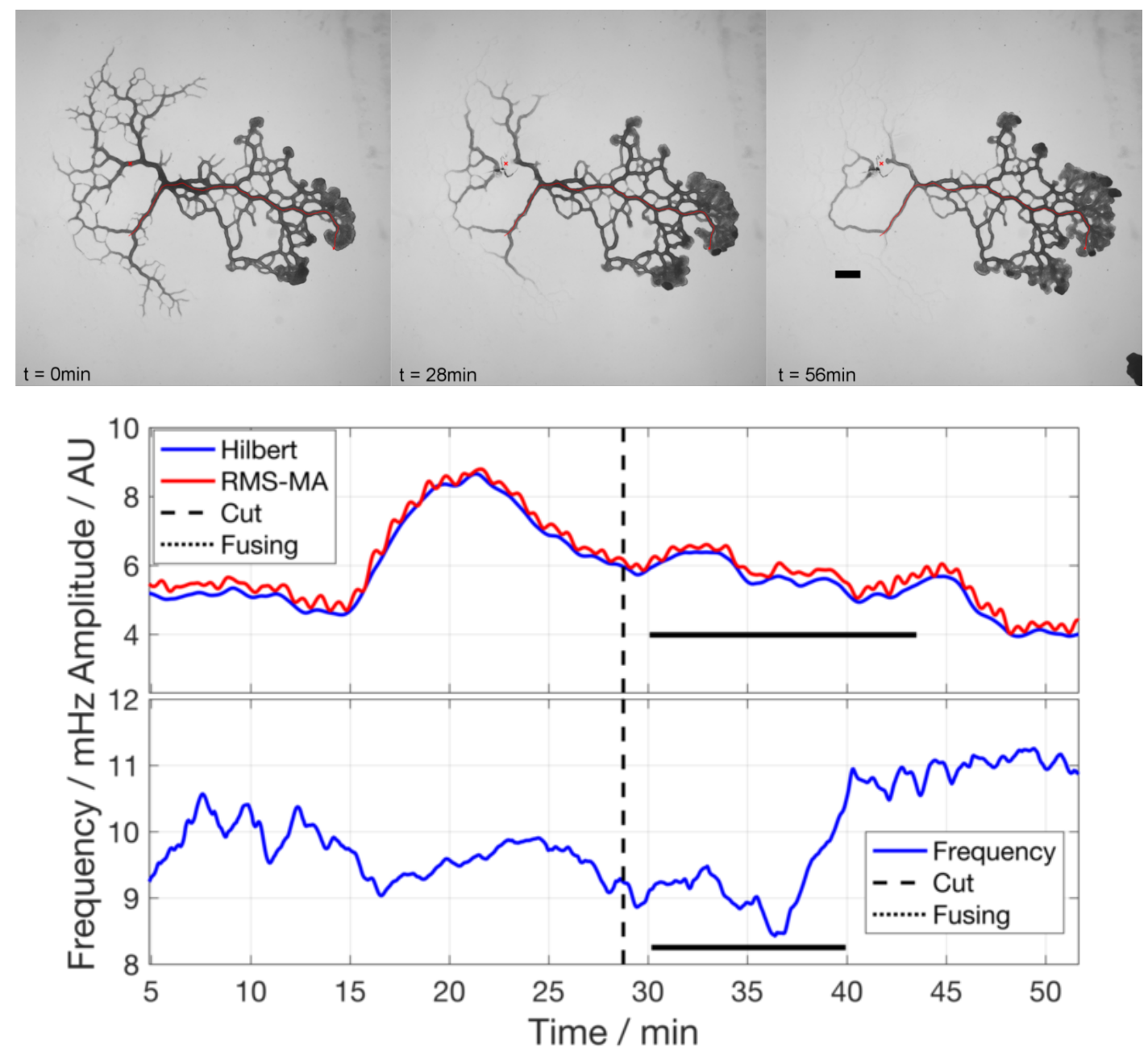

Figure A25: Here a section in the trailing part of the plasmodium is cut. Fan growth does occur, but hardly closes the gap as the neighbouring tubes are pruning to continue foraging in the distal fan region. Stalling is hardly noticeable and not evident from the amplitude graph. 

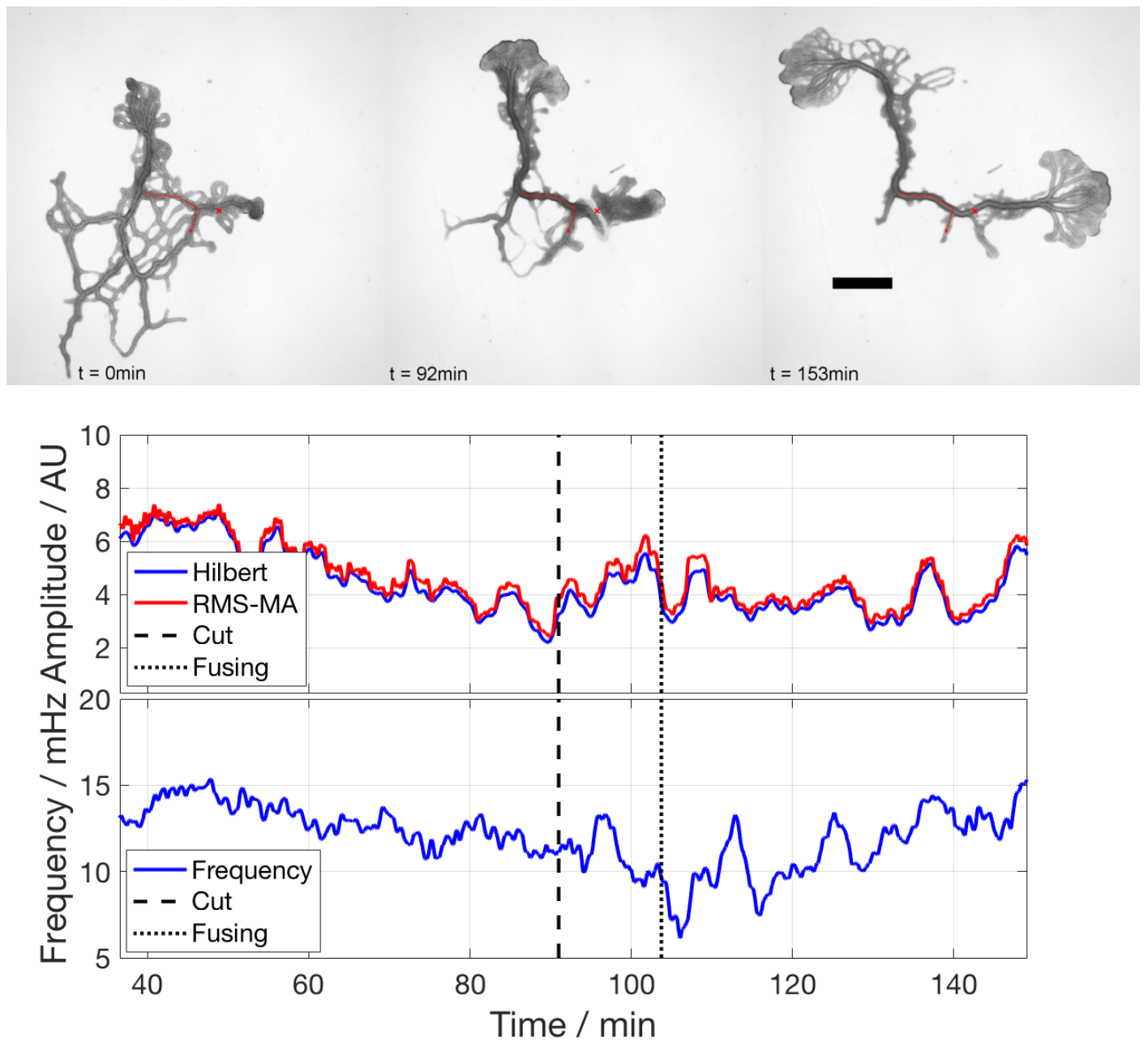

Figure A26: The small plasmodium in this experiment first undergoes a likely reaction to light, recovering into a structure made out of two fans connected by a thick tube. A cut is performed across the base of one of the fans. The tube, as well as the plasmodial sheet around it, rejoin quickly and the flow is re-established. There is no apparent stalling. 
A.7 Supplemental Figures for 'Spatial mapping reveals multi-step pattern of wound healing in Physarum polycephalum'
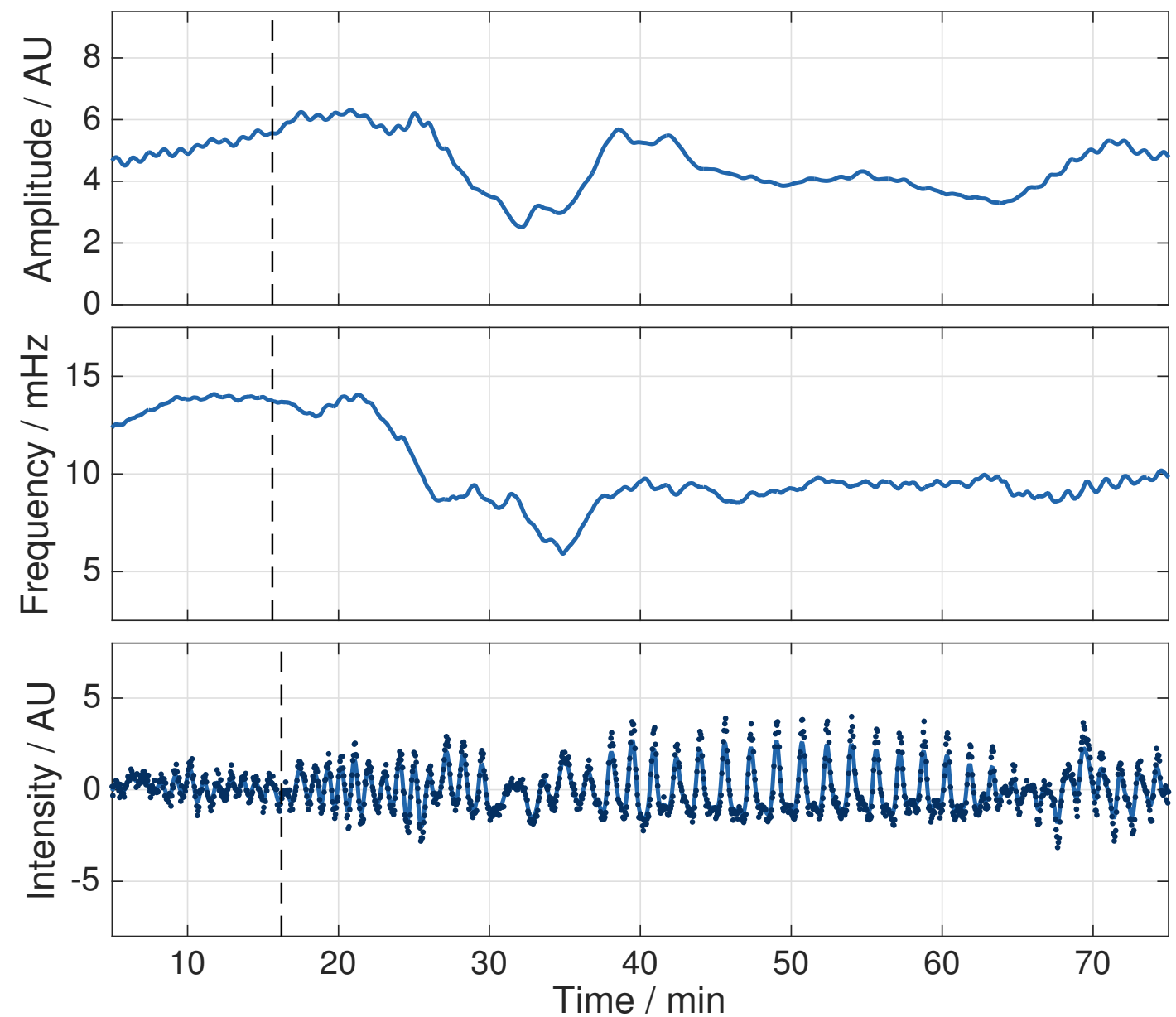

Figure A27: Analysis of averaged data from the whole network presented in the main text. The dotted line indicates the cutting event. Dotted points in the intensity plot show raw data and the line depicts Gaussian filtered data. Dynamics of small sub-network are lost in comparison to Fig. 5. 


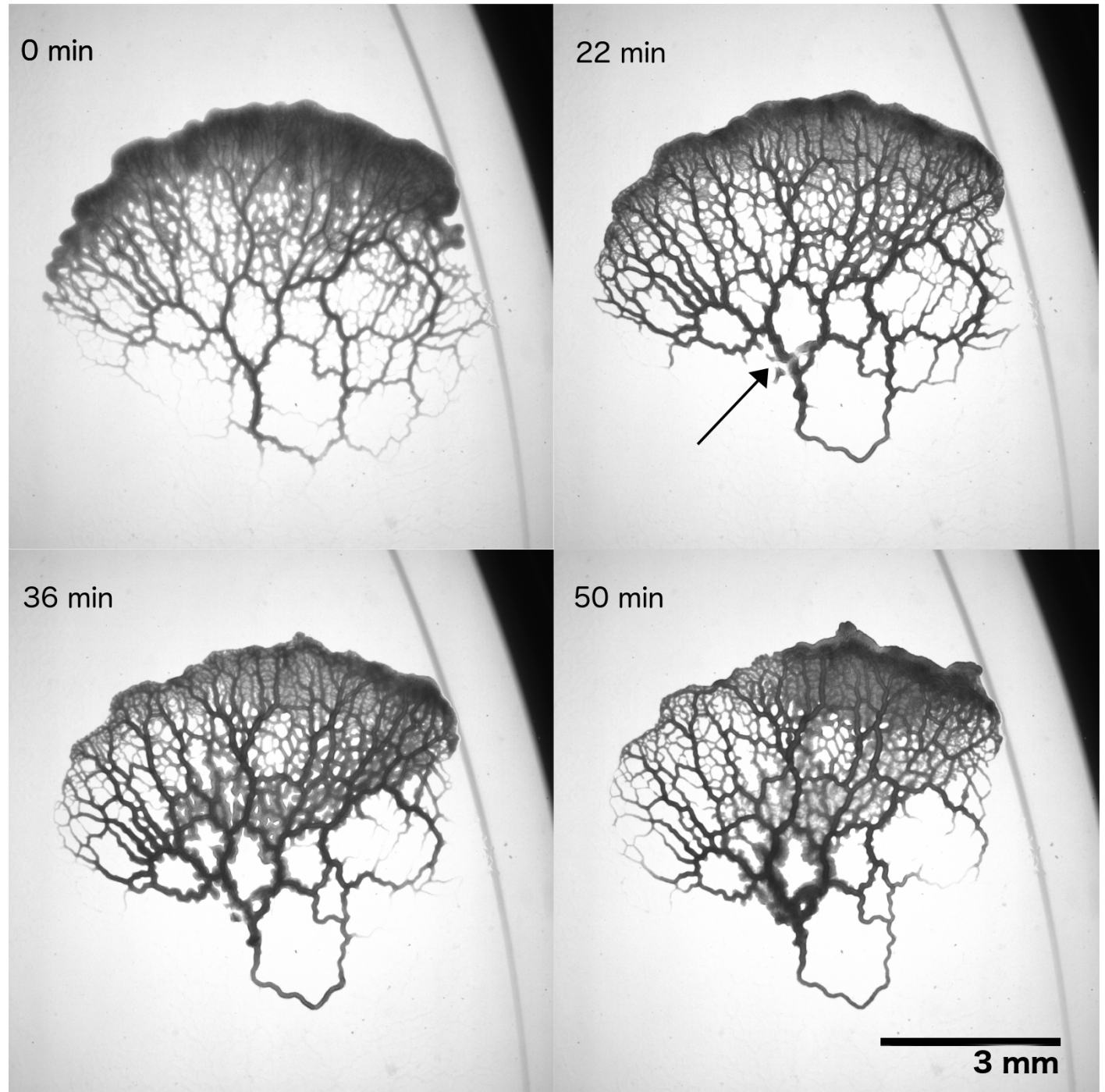

Figure A28: Material is transported towards the cut site (arrow). In the minutes after the cut, body mass accumulates along the general direction of the cut site and fans develop around it. 


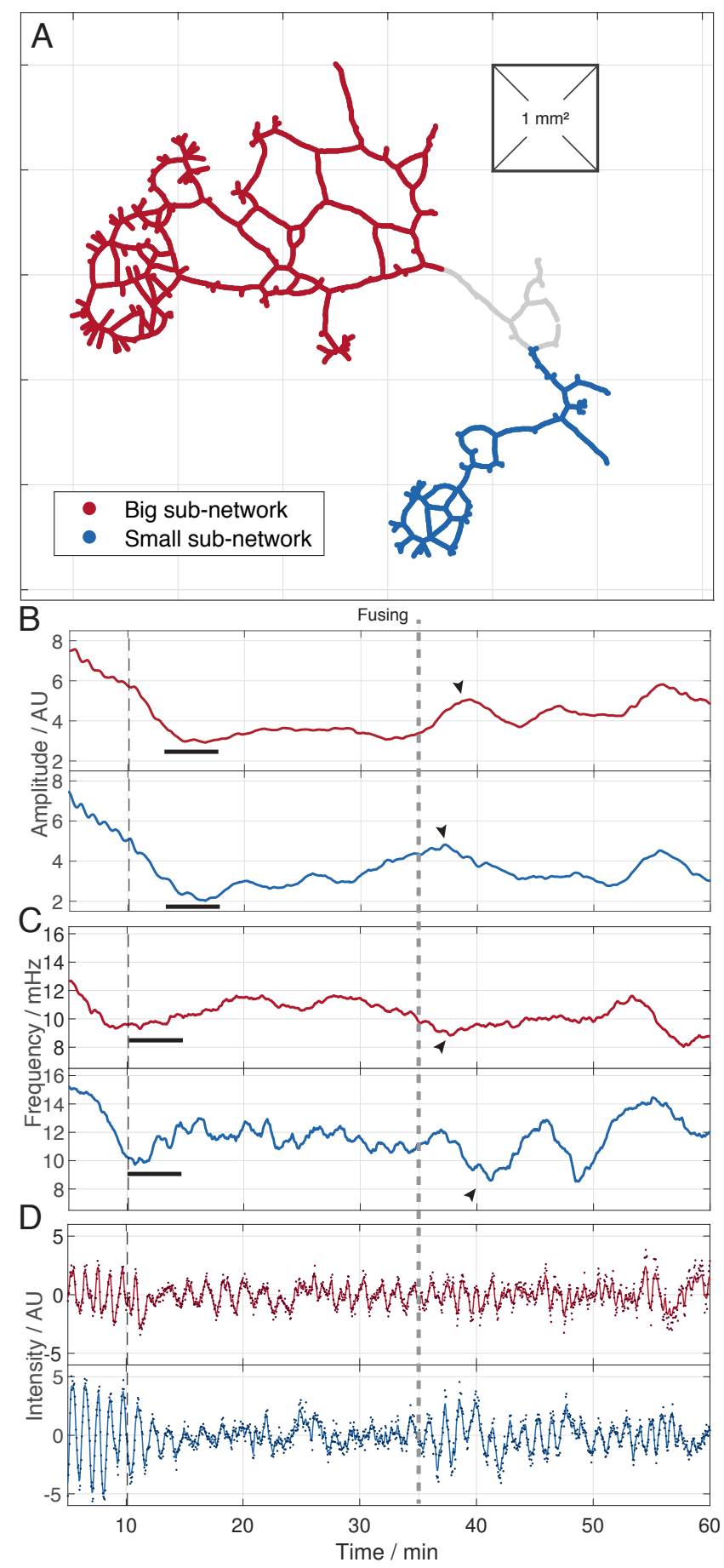

Figure A29: Full quantitative analysis of a second dataset (E3) for comparison. Oscillation parameters in the big- and small-sub-network depicted in (A) which result from the cut. Grey area (cut site) is not considered in the analysis. Time series of amplitude (B), frequency (C) and intensity (D) are averaged in the respective domains and compared; top : big sub-network, bottom: small sub-network. In each of these plots the black dashed line indicates the moment of the cut. The grey dashed line marks the time point of fusion. Black bars underline periods of stalling in (B) and (C). In (D) the red and blue solid lines represents the Gaussian filtered intensity (kernel width $=39$ s) and markers show raw averaged data. Black arrows indicate respective extremal peaks in the transition periods.

In comparison to Fig. 5 stalling is not as pronounced in frequency and occurs simultaneously in both sub-networks. Neither frequency or amplitude recover fully, but both are already declining pre-stimulus. The transition phase is visible after fusing with peaks in amplitude and decline in frequency. Network-spanning contractions are occurring in the big sub-network before fusion and are directed towards fan regions and the cut site. In general, the fan size at the cut site is comparatively small and reached maximal size shortly after fusion. 

A.8 Supplemental Movies for 'Spatial mapping reveals multi-step pattern of wound healing in Physarum polycephalum'

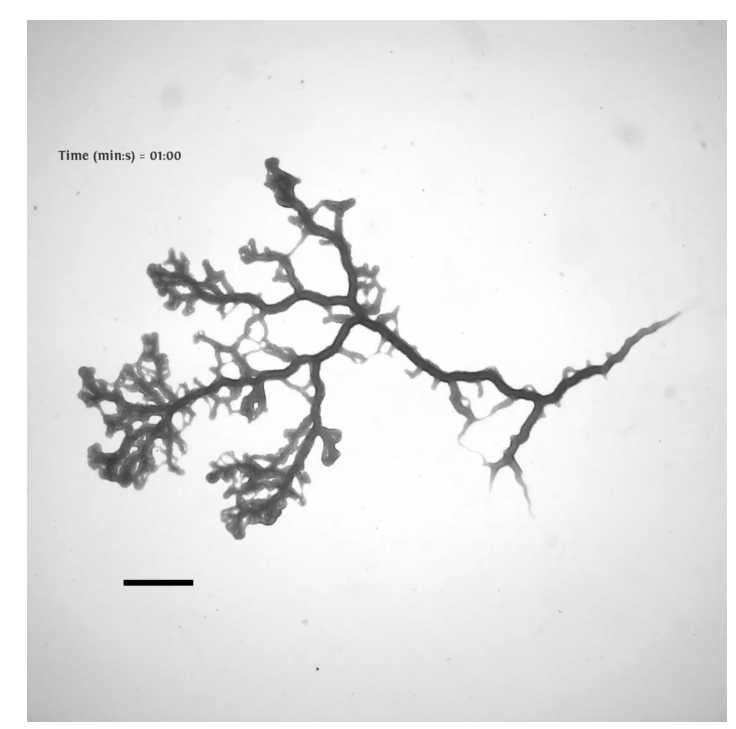

Movie M1: Bright field movie of the representative data set for wound healing. Note, that the cessation of oscillations in the top and bottom part (big and small sub-network) is on different time scales. Scale bar $=1 \mathrm{~mm}$.

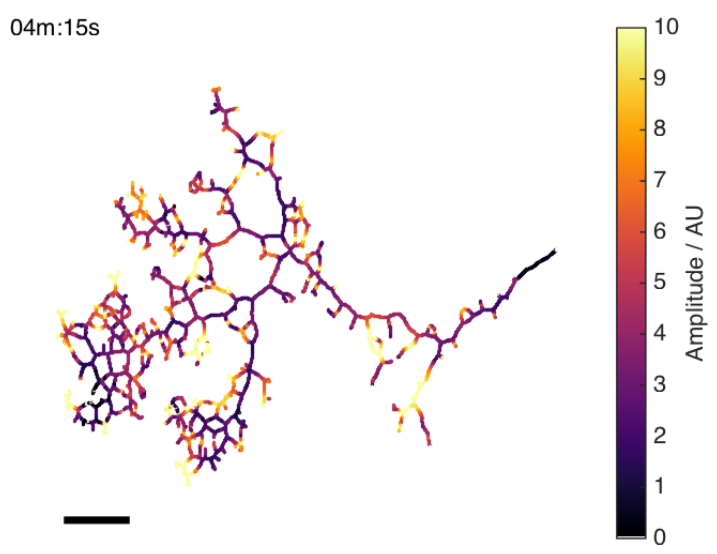

Movie M2: Movie of the oscillation amplitude spatially mapped across the network. Amplitudes below a threshold of 0.1 are omitted to visualize pruning. Due to detrending, the relative time point of cut appears shifted to $19 \mathrm{~min}$ and $50 \mathrm{~s}$. Scale bar $=1 \mathrm{~mm}$. 


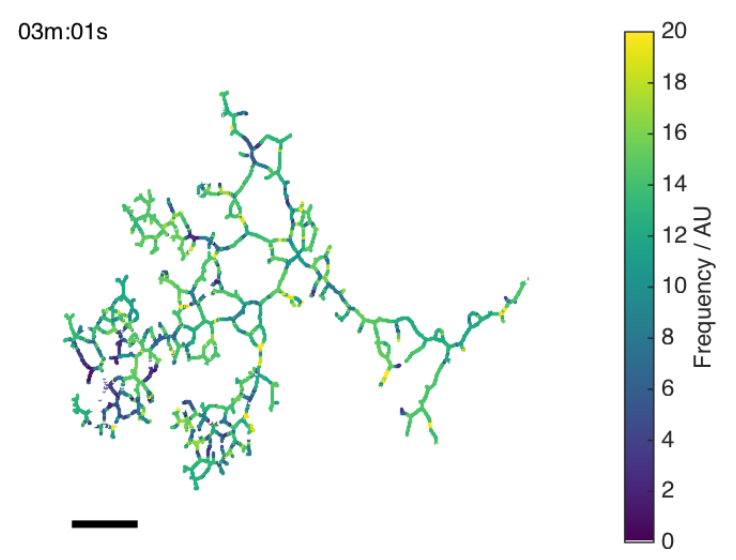

Movie M3: Spatially mapped oscillation frequency across the network. Mind that due to detrending, the relative time point of cut appears shifted to $19 \mathrm{~min}$ and $50 \mathrm{~s}$. Scale bar $=1 \mathrm{~mm}$.

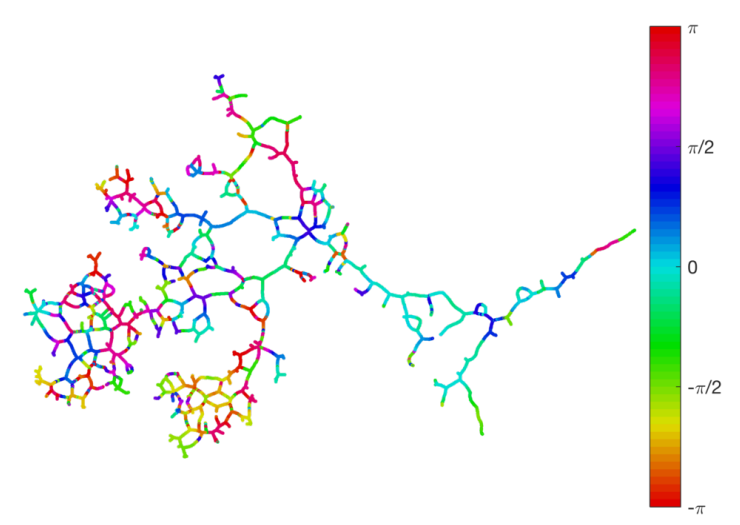

Movie M4: Spatially mapped phase pattern across the network with additional Gaussian smoothing in a 30px environment. Cut time is at $19 \mathrm{~min}$ and $50 \mathrm{~s}$. Mind change in phase waves from before cut, during stalling and during correlated phase. The cut itself is not visible in the phase pattern due to low amplitude residual slime and spatial smoothing. It is recommended to follow a single colour over a time span to guide ones eye. The network spans maximal $8.4 \mathrm{~mm}$ along the $\mathrm{x}$-axis. 


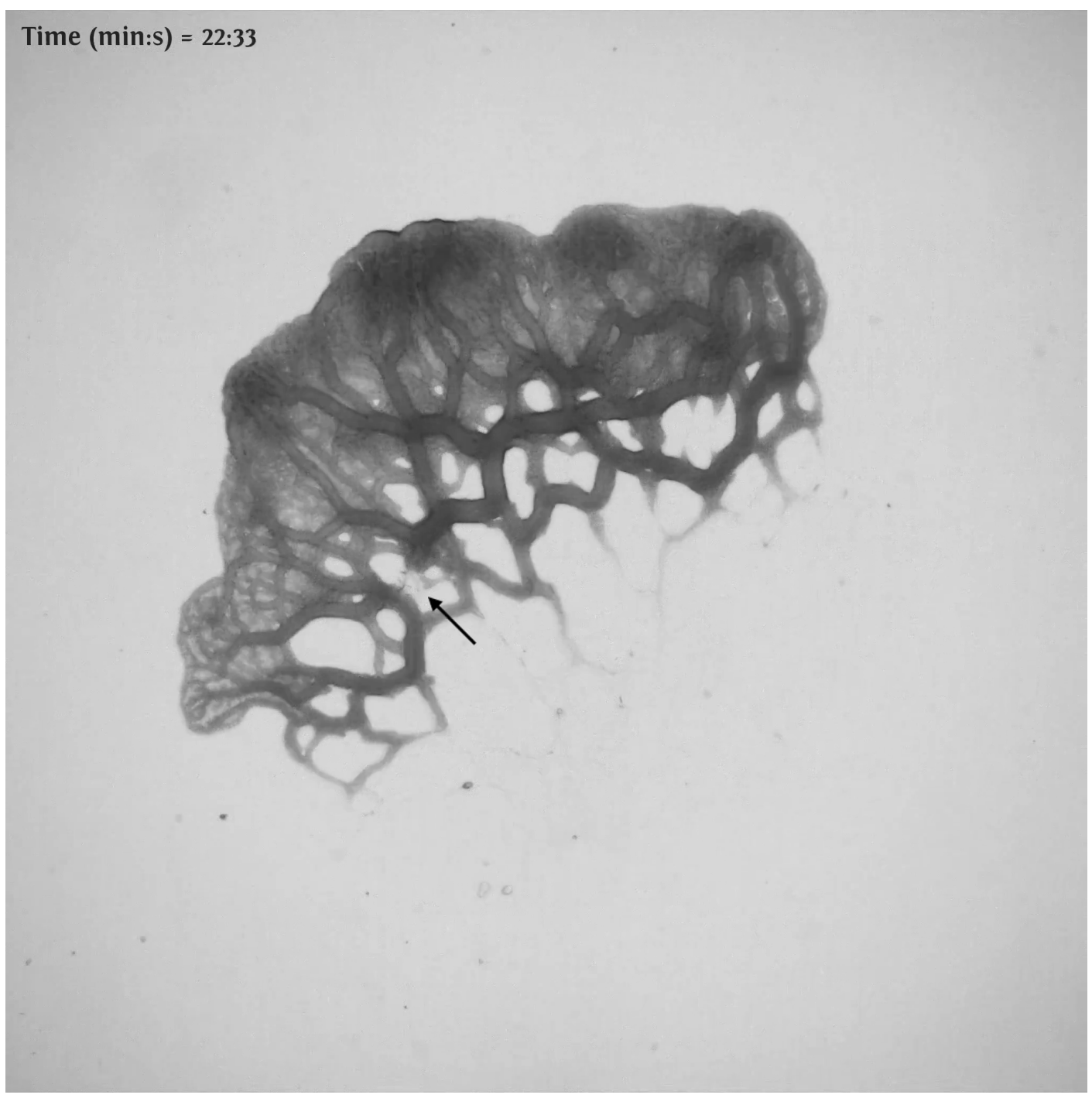

Movie M5: Bright field movie of E1 dataset showing severe stalling. Exemplary dataset for missing network morphology which makes network-based quantitative analysis impossible. Arrow marks cut location. Mind change in oscillation pattern, morphology and fan growth right after cut. Scale bar $=1 \mathrm{~mm}$. 

${ }^{1}$ D. C. Geary, The origin of mind: evolution of brain, cognition, and general intelligence, 1st ed, OCLC: ocm54906982 (American Psychological Association, Washington, DC, 2005).

${ }^{2}$ T. Nakagaki, H. Yamada, and Á. Tóth, "Maze-solving by an amoeboid organism," en, Nature 407, 470-470 (2000).

${ }^{3}$ A. Tero, S. Takagi, T. Saigusa, K. Ito, D. P. Bebber, M. D. Fricker, K. Yumiki, R. Kobayashi, and T. Nakagaki, "Rules for Biologically Inspired Adaptive Network Design," en, Science 327, 439-442 (2010).

${ }^{4}$ A. Dussutour, T. Latty, M. Beekman, and S. J. Simpson, "Amoeboid organism solves complex nutritional challenges," en, Proceedings of the National Academy of Sciences 107, 4607-4611 (2010).

${ }^{5}$ T. Saigusa, A. Tero, T. Nakagaki, and Y. Kuramoto, "Amoebae Anticipate Periodic Events," Phys. Rev. Lett. 100, 018101 (2008).

${ }^{6}$ C. R. Reid, T. Latty, A. Dussutour, and M. Beekman, "Slime mold uses an externalized spatial "memory" to navigate in complex environments," Proceedings of the National Academy of Sciences 109, 17490-17494 (2012).

${ }^{7}$ R. P. Boisseau, D. Vogel, and A. Dussutour, "Habituation in non-neural organisms: evidence from slime moulds," en, Proceedings of the Royal Society B: Biological Sciences 283, 20160446 (2016).

${ }^{8}$ H. W. Sauer, Developmental biology of Physarum, 11 (CUP Archive, 1982).

9 J. Baranetzki, "Influence de la lumière sur les plasmodia des Myxomycetes.," Mém. Soc. nat. Cherbourg 19, 321 (1876).

${ }^{10}$ W. F. Dove, J. Dee, S. Hatano, F. B. Haugli, and K.-E. WohlfarthBottermann, eds., The Molecular Biology of Physarum polycephalum, en (Springer New York, Boston, MA, 1986).

${ }^{11}$ C. Oettmeier, K. Brix, and H.-G. Döbereiner, "Physarum polycephalum - a new take on a classic model system," en, Journal of Physics D: Applied Physics 50, 413001 (2017). 
${ }^{12} \mathrm{~K}$. Hausmann, and R. Stiemerling, "In memoriam: Karl-Ernst Wohlfarth-Bottermann (1923-1997)," de, European Journal of Protistology 33, 452-459 (1997).

13P. Schaap, et al., "The Physarum polycephalum Genome Reveals Extensive Use of Prokaryotic Two-Component and Metazoan-Type Tyrosine Kinase Signaling," en, Genome Biology and Evolution 8, 109-125 (2016).

${ }^{14} \mathrm{~L}$. Eichinger, et al., "The genome of the social amoeba Dictyostelium discoideum," en, Nature 435, 43-57 (2005).

${ }^{15} \mathrm{C}$. Westendorf, J. Negrete, A. J. Bae, R. Sandmann, E. Bodenschatz, and C. Beta, "Actin cytoskeleton of chemotactic amoebae operates close to the onset of oscillations," en, Proceedings of the National Academy of Sciences 110, 3853-3858 (2013).

${ }^{16}$ R. L. Kincaid, and T. E. Mansour, "Cyclic 3',5'-AMP phosphodiesterase in Physarum polycephalum I. Chemotaxis toward inhibitors and cyclic nucleotides," en, Biochimica et Biophysica Acta (BBA) - General Subjects 588, 332-341 (1979).

${ }^{17}$ C. Westendorf, C. J. Gruber, K. Schnitzer, S. Kraker, and M. Grube, "Quantitative comparison of plasmodial migration and oscillatory properties across different slime molds," en, Journal of Physics D: Applied Physics 51, 344001 (2018).

${ }^{18}$ E. Guttes, S. Guttes, and H. P. Rusch, "Morphological observations on growth and differentiation of Physarum polycephalum grown in pure culture," en, Developmental Biology 3, 588-614 (1961).

${ }^{19}$ D. Vogel, J. Gautrais, A. Perna, D. J. T. Sumpter, J.-L. Deneubourg, and A. Dussutour, "Transition from isotropic to digitated growth modulates network formation in Physarum polycephalum," en, Journal of Physics D: Applied Physics 50, 014002 (2017).

${ }^{20} \mathrm{~N}$. Kamiya, "The Rate of the Protoplasmic Flow in the Myxomycete Plasmodium. I," en, CYTOLOGIA 15, 183-193 (1950).

${ }^{21}$ S. Yoshiyama, M. Ishigami, A. Nakamura, and K. Kohama, "Calcium wave for cytoplasmic streaming of Physarum polycephalum," en, Cell Biology International (2009) 10.1042/CBI20090158.

${ }^{22}$ K. Kohama, H. Takano-Ohmuro, T. Tanaka, Y. Yamaguchi, and T. Kohama, "Isolation and characterization of myosin from amoebae of Physarum polycephalum," eng, The Journal of Biological Chemistry 261, 8022-8027 (1986). 
${ }^{23}$ Y. Yoshimoto, F. Matsumura, and N. Kamiya, "Simultaneous oscillations of Ca2+ efflux and tension generation in the permealized plasmodial strand of physarum," en, Cell Motility 1, 433-443 (1981).

${ }^{24} \mathrm{~T}$. P. Stossel, "Contribution of actin to the structure of the cytoplasmic matrix," en, The Journal of Cell Biology 99, 15s-21 (1984).

${ }^{25} \mathrm{~W}$. Stockem, and K. Brix, "Analysis of Microfilament Organization and Contractile Activities in Physarum," en, in International Review of Cytology, Vol. 149 (Elsevier, 1994), pp. 145-215.

${ }^{26}$ A. Fessel, C. Oettmeier, K. Wechsler, and H.-G. Döbereiner, "Indentation analysis of active viscoelastic microplasmodia of P. polycephalum," en, Journal of Physics D: Applied Physics 51, 024005 (2018).

${ }^{27} \mathrm{~K}$. E. Wohlfarth-Bottermann, "Oscillatory contraction activity in Physarum," The Journal of Experimental Biology 81, 15 (1979).

${ }^{28}$ K. Alim, G. Amselem, F. Peaudecerf, M. P. Brenner, and A. Pringle, "Random network peristalsis in Physarum polycephalum organizes fluid flows across an individual," Proceedings of the National Academy of Sciences 110, 13306-13311 (2013).

${ }^{29} \mathrm{~J}$. A. Kitching, and M. H. Pirenne, "The influence of low tensions of oxygen on the protoplasmic streaming of myxomycetes," en, Journal of Cellular and Comparative Physiology 16, 131-133 (1940).

${ }^{30} \mathrm{~K}$. E. Wohlfarth-Bottermann, "Oscillating contractions in protoplasmic strands of Physarum: simultaneous tensiometry of longitudinal and radial rhythms, periodicity analysis and temperature dependence," The Journal of Experimental Biology 67, 49 (1977).

${ }^{31} \mathrm{~K}$. Alim, N. Andrew, A. Pringle, and M. P. Brenner, "Mechanism of signal propagation in Physarum polycephalum," en, Proceedings of the $\mathrm{Na}-$ tional Academy of Sciences 114, 5136-5141 (2017).

${ }^{32} \mathrm{~S}$. G. Proskurin, and T. I. Avsievich, "Spectral analysis of self-oscillating motility in an isolated plasmodial strand of Physarum polycephalum," en, Biophysics 59, 928-934 (2014).

${ }^{33}$ S. Marbach, K. Alim, N. Andrew, A. Pringle, and M. P. Brenner, "Pruning to Increase Taylor Dispersion in Physarum polycephalum Networks," en, Physical Review Letters 117 (2016) 10.1103/PhysRevLett.117.178103.

${ }^{34} \mathrm{~N}$. Kamiya, "THE CONTROL OF PROTOPLASMiC STREAMING," en, Science 92, 462-463 (1940). 
${ }^{35}$ N. Kamiya, "Physical and Chemical Basis of Cytoplasmic Streaming," en, Annual Review of Plant Physiology 32, 205-236 (1981).

${ }^{36}$ D. Akita, I. Kunita, M. D. Fricker, S. Kuroda, K. Sato, and T. Nakagaki, "Experimental models for Murray's law," en, Journal of Physics D: Applied Physics 50, 024001 (2017).

37J.-D. Julien, and K. Alim, "Oscillatory fluid flow drives scaling of contraction wave with system size," en, Proceedings of the National Academy of Sciences 115, 10612-10617 (2018).

${ }^{38}$ O. L. Lewis, S. Zhang, R. D. Guy, and J. C. del Alamo, "Coordination of contractility, adhesion and flow in migrating Physarum amoebae," en, Journal of The Royal Society Interface 12, 20141359-20141359 (2015).

${ }^{39}$ M. Radszuweit, H. Engel, and M. Bär, "A model for oscillations and pattern formation in protoplasmic droplets of Physarum polycephalum," en, The European Physical Journal Special Topics 191, 159-172 (2010).

${ }^{40}$ M. Radszuweit, H. Engel, and M. Bär, "An Active Poroelastic Model for Mechanochemical Patterns in Protoplasmic Droplets of Physarum polycephalum," en, PLoS ONE 9, edited by H. Jönsson, e99220 (2014).

${ }^{41} \mathrm{~V}$. A. Teplov, "Role of mechanics in the appearance of oscillatory instability and standing waves of the mechanochemical activity in the Physarum polycephalum plasmodium," Journal of Physics D: Applied Physics 50, 213002 (2017).

${ }^{42}$ W. D. Gray, "The Effect of Light on the Fruiting of Myxomycetes, " American Journal of Botany 25, 511-522 (1938).

${ }^{43} \mathrm{C}$. Starostzik, and W. Marwan, "A photoreceptor with characteristics of phytochrome triggers sporulation in the true slime mould Physarum polycephalum," en, FEBS Letters 370, 146-148 (1995).

44W. Marwan, "Chapter 20 Photomovement and photomorphogenesis in Physarum polycephalum: targeting of cytoskeleton and gene expression by light," en, in Comprehensive Series in Photosciences, Vol. 1 (Elsevier, 2001), pp. 561-587.

${ }^{45}$ B. Poetsch, T. Schreckenbach, and A. K. Werenskiold, "Photomorphogenesis in Physarum polycephalum. Temporal expression pattern of actin, alpha- and beta-tubulin, " eng, European Journal of Biochemistry 179, 141-146 (1989).

${ }^{46}$ X. Yu, H. Liu, J. Klejnot, and C. Lin, "The Cryptochrome Blue Light Receptors," en, The Arabidopsis Book 8, e0135 (2010). 
${ }^{47}$ N. Ozturk, C. P. Selby, D. Zhong, and A. Sancar, "Mechanism of Photosignaling by Drosophila Cryptochrome: ROLE OF THE REDOX STATUS OF THE FLAVIN CHROMOPHORE," en, Journal of Biological Chemistry 289, 4634-4642 (2014).

${ }^{48}$ E. A. Gorostiza, J. Colomb, and B. Brembs, "A decision underlies phototaxis in an insect," en, Open Biology 6, 160229 (2016).

${ }^{49}$ P. G. Koehler, H. R. Agee, N. C. Leppla, and R. S. Patterson, "Spectral Sensitivity and Behavioral Response to Light Quality in the German Cockroach (Dictyoptera: Blattellidae)," en, Annals of the Entomological Society of America 80, 820-822 (1987).

${ }^{50} \mathrm{G}$. Jékely, "Evolution of phototaxis," en, Philosophical Transactions of the Royal Society B: Biological Sciences 364, 2795-2808 (2009).

${ }^{51} \mathrm{~K}$. Drescher, R. E. Goldstein, and I. Tuval, "Fidelity of adaptive phototaxis," en, Proceedings of the National Academy of Sciences 107, 1117111176 (2010).

${ }^{52} \mathrm{~L}$. Rakoczy, "Effect of Blue Light on Metabolic Processes, Development and Movement in True Slime Molds," in The Blue Light Syndrome, edited by H. Senger, (Springer Berlin Heidelberg, Berlin, Heidelberg, 1980), pp. 570-583.

53J. W. Daniel, "Light-Induced Synchronous Sporulation of a Myxomycete-The Relation of Initial Metabolic Changes to the Establishment of a New Cell State," en, in Cell Synchrony (Elsevier, 1966), pp. 117152.

54T. Ueda, Y. Mori, T. Nakagaki, and Y. Kobatake, "ACTION SPECTRA FOR SUPEROXIDE GENERATION AND UV AND VISIBLE LIGHT PHOTOAVOIDANCE IN PLASMODIA OF Physarum polycephalum," en, Photochemistry and Photobiology 48, 705-709 (1988).

55J. W. Daniel, and H. H. Baldwin, "Chapter 2 Methods of Culture for Plasmodial Myxomycetes," en, in Methods in Cell Biology, Vol. 1 (Elsevier, 1964), pp. 9-41.

${ }^{56} \mathrm{~J}$. Bialczyk, and L. Rakoczy, "Phototaxis of the Myxomycetes. Behaviour of old plasmodia in white light," eng, Bulletin De l'Academie Polonaise Des Sciences. Serie Des Sciences Biologiques 23, 571-575 (1975).

${ }^{57}$ M. Hato, T. Ueda, K. Kurihara, and Y. Kobatake, "Phototaxis in True Slime Mold Physarum polycephalum," en, Cell Structure and Function 1, 269-278 (1976). 
${ }^{58} \mathrm{~J}$. Bialczyk, and L. Rakoczy, "Phototaxis in the Myxomycetes. Migration of the young plasmodia in white light," eng, Bulletin De l'Academie Polonaise Des Sciences. Serie Des Sciences Biologiques 22, 871-874 (1974).

${ }^{59}$ Y. Mori, K. Matsumoto, T. Ueda, and Y. Kobatake, "Spatio-temporal organization of intracellular ATP content and oscillation patterns in response to blue light byPhysarum polycephalum, " en, Protoplasma 135, 31-37 (1986).

${ }^{60} \mathrm{~T}$. Nakagaki, S. Umemura, Y. Kakiuchi, and T. Ueda, "Action Spectrum for Sporulation and Photoavoidance in the Plasmodium of Physarum polycephalum, as Modified Differentially by Temperature and Starvation," en, Photochemistry and Photobiology 64, 859-862 (1996).

${ }^{61}$ I. Block, and K. Wohlfarthbottermann, "Blue light as a medium to influence oscillatory contraction frequency in physarum, " en, Cell Biology International Reports 5, 73-81 (1981).

${ }^{62} \mathrm{~K}$. Wohlfarthbottermann, and I. Block, "The pathway of photosensory transduction in Physarum polycephalum, " en, Cell Biology International Reports 5, 365-373 (1981).

${ }^{63} \mathrm{~K}$. Wohlfarth-Bottermann, "Tensiometric demonstration of endogenous, oscillating contractions in plasmodia of Physarum polycephalum," en, Zeitschrift für Pflanzenphysiologie 76, 14-27 (1975).

${ }^{64 J}$. D. Huizinga, and W. J. E. P. Lammers, "Gut peristalsis is governed by a multitude of cooperating mechanisms," en, American Journal of Physiology-Gastrointestinal and Liver Physiology 296, G1-G8 (2009).

${ }^{65}$ P. A. Stewart, and B. T. Stewart, "Protoplasmic movement in slime mold plasmodia: The diffusion drag force hypothesis," en, Experimental Cell Research 17, 44-58 (1959).

${ }^{66} \mathrm{M}$. Iima, and T. Nakagaki, "Peristaltic transport and mixing of cytosol through the whole body of Physarum plasmodium," en, Mathematical Medicine and Biology 29, 263-281 (2012).

${ }^{67}$ O. L. Lewis, and R. D. Guy, "Analysis of peristaltic waves and their role in migrating Physarum plasmodia," en, Journal of Physics D: Applied Physics 50, 284001 (2017).

${ }^{68} \mathrm{M}$. Li, and J. G. Brasseur, "Non-steady peristaltic transport in finitelength tubes," en, Journal of Fluid Mechanics 248, 129 (1993). 
${ }^{69}$ A. H. Shapiro, M. Y. Jaffrin, and S. L. Weinberg, "Peristaltic pumping with long wavelengths at low Reynolds number," en, Journal of Fluid Mechanics 37, 799-825 (1969).

${ }^{70} \mathrm{C}$. Nations, and J. L. McCarthy, "Growth of white microplasmodia of Physarum polycephalum," en, Comparative Biochemistry and Physiology Part A: Physiology 78, 459-462 (1984).

${ }^{71} \mathrm{~W}$. Alt, and M. Dembo, "Cytoplasm dynamics and cell motion: twophase flow models," en, Mathematical Biosciences 156, 207-228 (1999).

${ }^{72}$ R. Swaminathan, C. Hoang, and A. Verkman, "Photobleaching recovery and anisotropy decay of green fluorescent protein GFP-S65t in solution and cells: cytoplasmic viscosity probed by green fluorescent protein translational and rotational diffusion," en, Biophysical Journal 72, 1900-1907 (1997).

73J. Sambrook, and D. W. Russell, Molecular cloning: a laboratory manual, 3rd ed (Cold Spring Harbor Laboratory Press, Cold Spring Harbor, N.Y, 2001).

${ }^{74}$ A. Fessel, C. Oettmeier, E. Bernitt, N. C. Gauthier, and H.-G. Döbereiner, "Physarum polycephalum Percolation as a Paradigm for Topological Phase Transitions in Transportation Networks," Phys. Rev. Lett. 109, 078103 (2012).

$75 \mathrm{~F}$. K. Bäuerle, M. Kramar, and K. Alim, "Spatial mapping reveals multistep pattern of wound healing in Physarum polycephalum," en, Journal of Physics D: Applied Physics 50, 434005 (2017).

${ }^{76}$ K.-S. Chuang, H.-L. Tzeng, S. Chen, J. Wu, and T.-J. Chen, "Fuzzy Cmeans clustering with spatial information for image segmentation," en, Computerized Medical Imaging and Graphics 30, 9-15 (2006).

${ }^{77}$ W. Abu-Ain, S. N. H. S. Abdullah, B. Bataineh, T. Abu-Ain, and K. Omar, "Skeletonization Algorithm for Binary Images," en, Procedia Technology 11, 704-709 (2013).

${ }^{78}$ T. Nakagaki, H. Yamada, and T. Ueda, "Interaction between cell shape and contraction pattern in the Physarum plasmodium," en, Biophysical Chemistry 84, 195-204 (2000).

${ }^{79}$ T. Ueda, M. Muratsugu, K. Kurihara, and Y. Kobatake, "Chemotaxis in Physarum polycephalum," en, Experimental Cell Research 100, 337-344 (1976). 
${ }^{80}$ A. C. Durham, "Control of chemotaxis in Physarum polycephalum," en, The Journal of Cell Biology 69, 218-223 (1976).

${ }^{81}$ I. Chet, A. Naveh, and Y. Henis, "Chemotaxis of Physarum polycephalum towards Carbohydrates, Amino Acids and Nucleotides," en, Journal of General Microbiology 102, 145-148 (1977).

${ }^{82}$ Y. Miyake, H. Tada, M. Yano, and H. Shimizu, "Relationship between intracellular period modulation and external environment change in Physarum plasmodium," eng, Cell Structure and Function 19, 363-370 (1994).

83Z. Hejnowicz, and K. E. Wohlfarth-Bottermann, "Propagated waves induced by gradients of physiological factors within plasmodia ofPhysarum polycephalum," en, Planta 150, 144-152 (1980).

${ }^{84 T}$. Hirose, T. Ueda, and Y. Kobatake, "Changes in Intracellular pH Accompanying Chemoreception in the Plasmodia of Physarum polycephalum," en, Microbiology 128, 2647-2651 (1982).

${ }^{85} \mathrm{~A}$. McClory, and J. Coote, "The chemotactic response of the myxomycete Physarum polyhcepalum to amino acids, cyclic nucleotides and folic acid," en, FEMS Microbiology Letters 26, 195-200 (1985).

${ }^{86} \mathrm{~K}$. Matsumoto, T. Ueda, and Y. Kobatake, "Reversal of thermotaxis with oscillatory stimulation in the plasmodium of Physarum polycephalum," Journal of Theoretical Biology 131, 175-182 (1988).

${ }^{87}$ A. Takamatsu, T. Yamamoto, and T. Fujii, "Spontaneous switching of frequency-locking by periodic stimulus in oscillators of plasmodium of the true slime mold," Biosystems 76, 133-140 (2004).

${ }^{88} \mathrm{~K}$. E. Wohlfarth-Bottermann, and I. Block, "Function of cytoplasmic flow in photosensory transduction and phase regulation of contractile activities in Physarum," eng, Cold Spring Harbor Symposia on Quantitative Biology 46 Pt 2, 563-568 (1982).

${ }^{89} \mathrm{~T}$. Nakagaki, H. Yamada, and T. Ueda, "Modulation of cellular rhythm and photoavoidance by oscillatory irradiation in the Physarum plasmodium," Biophysical Chemistry 82, 23-28 (1999).

${ }^{90} \mathrm{~N}$. Kamiya, and Y. Yoshimoto, "Dynamic characteristics of the cytoplasm. A study on the plasmodial strand of a myxomycete," in Aspects of Cellular and Molecular Physiology (Univ. Tokyo Press, Tokyo, 1972), pp. 167-189. 
${ }^{91}$ Y. Yoshimoto, and N. Kamiya, "Studies on contraction rhythm of the plasmodial strand IV. Site of active oscillation in an advancing plasmodium," en, Protoplasma 95, 123-133 (1978).

${ }^{92} \mathrm{M}$. Cielawska, and J. Koodziejczyk, "The influence of dissection and of the contact with glass on the contractile activity of plasmodial strands," en, Cell Biology International Reports 8, 813-821 (1984).

${ }^{93} \mathrm{U}$. Achenbach, and K. E. Wohlfarth-Bottermann, "Synchronization and signal transmission in protoplasmic strands of Physarum: Effects of externally applied substances and mechanical influences," en, Planta 151, 574-583 (1981).

${ }^{44}$ S. Kuroda, S. Takagi, T. Nakagaki, and T. Ueda, "Allometry in Physarum plasmodium during free locomotion: size versus shape, speed and rhythm," en, Journal of Experimental Biology 218, 3729-3738 (2015).

${ }^{95} \mathrm{~N}$. Cohen, and J. H. Boyle, "Swimming at low Reynolds number: a beginners guide to undulatory locomotion," en, Contemporary Physics 51, 103-123 (2010).

${ }^{96}$ J. M. Davidenko, A. V. Pertsov, R. Salomonsz, W. Baxter, and J. Jalife, "Stationary and drifting spiral waves of excitation in isolated cardiac muscle," en, Nature 355, 349-351 (1992).

${ }^{97}$ S. Camalet, F. Jülicher, and J. Prost, "Self-Organized Beating and Swimming of Internally Driven Filaments," en, Physical Review Letters 82, 1590-1593 (1999).

${ }^{98}$ D. Bell-Pedersen, V. M. Cassone, D. J. Earnest, S. S. Golden, P. E. Hardin, T. L. Thomas, and M. J. Zoran, "Circadian rhythms from multiple oscillators: lessons from diverse organisms," en, Nature Reviews Genetics 6 , 544-556 (2005).

${ }^{99} \mathrm{~S}$. Kondo, and T. Miura, "Reaction-Diffusion Model as a Framework for Understanding Biological Pattern Formation," en, Science 329, 16161620 (2010).

${ }^{100}$ R. M. Alexander, "Optimization and gaits in the locomotion of vertebrates," en, Physiological Reviews 69, 1199-1227 (1989).

${ }^{101} \mathrm{M}$. W. Berchtold, H. Brinkmeier, and M. Müntener, "Calcium Ion in Skeletal Muscle: Its Crucial Role for Muscle Function, Plasticity, and Disease," en, Physiological Reviews 80, 1215-1265 (2000). 
${ }^{102}$ S. Berri, J. H. Boyle, M. Tassieri, I. A. Hope, and N. Cohen, "Forward locomotion of the nematode $C$. elegans is achieved through modulation of a single gait," en, HFSP Journal 3, 186-193 (2009).

${ }^{103}$ N. Osterman, and A. Vilfan, "Finding the ciliary beating pattern with optimal efficiency," en, Proceedings of the National Academy of Sciences 108, 15727-15732 (2011).

104Y. Katsu-Kimura, F. Nakaya, S. A. Baba, and Y. Mogami, "Substantial energy expenditure for locomotion in ciliates verified by means of simultaneous measurement of oxygen consumption rate and swimming speed," en, Journal of Experimental Biology 212, 1819-1824 (2009).

${ }^{105} \mathrm{~J}$. D. Huizinga, et al., "The origin of segmentation motor activity in the intestine," en, Nature Communications 5 (2014) 10.1038/ncomms4326.

${ }^{106}$ G. Saggiorato, L. Alvarez, J. F. Jikeli, U. B. Kaupp, G. Gompper, and J. Elgeti, "Human sperm steer with second harmonics of the flagellar beat," en, Nature Communications 8 (2017) 10.1038/s41467-017-01462-y.

${ }^{107}$ O. Mahrenholtz, M. Mank, and R. Zimmermann, "The influence of wave form on peristaltic transport," Biorheology 15, 501-510 (1978).

${ }^{108}$ A. Oron, S. H. Davis, and S. G. Bankoff, "Long-scale evolution of thin liquid films," en, Reviews of Modern Physics 69, 931-980 (1997).

${ }^{109}$ G. Salbreux, G. Charras, and E. Paluch, "Actin cortex mechanics and cellular morphogenesis," en, Trends in Cell Biology 22, 536-545 (2012).

${ }^{110}$ E. M. Purcell, "Life at low Reynolds number," en, American Journal of Physics 45, 3-11 (1977).

${ }^{111}$ D. Takagi, and N. J. Balmforth, "Peristaltic pumping of viscous fluid in an elastic tube," en, Journal of Fluid Mechanics 672, 196-218 (2011).

${ }^{112}$ A. Pandey, S. Karpitschka, C. H. Venner, and J. H. Snoeijer, "Lubrication of soft viscoelastic solids, " en, Journal of Fluid Mechanics 799, 433-447 (2016).

${ }^{113}$ S. Alonso, M. Radszuweit, H. Engel, and M. Bär, "Mechanochemical pattern formation in simple models of active viscoelastic fluids and solids," en, Journal of Physics D: Applied Physics 50, 434004 (2017).

${ }^{114}$ S. Takagi, and T. Ueda, "Annihilation and creation of rotating waves by a local light pulse in a protoplasmic droplet of the Physarum plasmodium," en, Physica D: Nonlinear Phenomena 239, 873-878 (2010). 
${ }^{115}$ S. Kang, et al., "Between a Pod and a Hard Test: The Deep Evolution of Amoebae," en, Molecular Biology and Evolution 34, edited by T. Leitner, 2258-2270 (2017).

${ }^{116}$ S. Feng, and P. N. Sen, "Percolation on Elastic Networks: New Exponent and Threshold," en, Physical Review Letters 52, 216-219 (1984).

${ }^{117}$ M. Rea-Maminta, N. Dagamac, F. Huyop, R. Wahab, and T. dela Cruz, "Comparative diversity and heavy metal biosorption of myxomycetes from forest patches on ultramafic and volcanic soils," en, Chemistry and Ecology 31, 741-753 (2015).

${ }^{118}$ D. A. Zhulidov, R. D. Robarts, A. V. Zhulidov, O. V. Zhulidova, D. A. Markelov, V. A. Rusanov, and J. V. Headley, "Zinc accumulation by the slime mold Fuligo septica (L.) Wiggers in the former Soviet Union and North Korea," eng, Journal of Environmental Quality 31, 1038-1042 (2002).

${ }^{119}$ H. Nobach, Gegen Wavelets \& Co: Vorteile klassischer Signal- und Datenverarbeitungsverfahren in der optischen Strömungsmesstechnik, ger, Messtechnik und Sensorik, OCLC: 188239169 (Shaker, Aachen, 2007).

${ }^{120} \mathrm{~L}$. Marple, "Computing the discrete-time "analytic"signal via FFT," IEEE Transactions on Signal Processing 47, 2600-2603 (1999).

${ }^{121}$ W. Naib-Majani, V. A. Teplov, and Z. Baranowski, "Morphology and Visco-Elastic Properties of Physarum Strands During the Steady-State of Their Contractile Behavior," in Cell Dynamics, Vol. 1, edited by M. Tazawa, (Springer Vienna, Vienna, 1988), pp. 57-63.

${ }^{122} \mathrm{~B}$. Audoly, and Y. Pomeau, Elasticity and geometry: from hair curls to the non-linear response of shells (Oxford University Press, Oxford ; New York, 2010).

${ }^{123}$ J. L. Sanders, "Nonlinear theories for thin shells," en, Quarterly of Applied Mathematics 21, 21-36 (1963). 

Colophon

This document was typeset using the typographical look-and-feel classicthesis developed by André Miede. The style was inspired by Robert Bringhurst's seminal book on typography "The Elements of Typographic Style". classicthesis is available for both $\mathrm{ET}_{\mathrm{E}} \mathrm{X}$ and $\mathrm{LYX}$ :

https://bitbucket.org/amiede/classicthesis/

Final Version as of December 19, 2019 (classicthesis version 0.5). 